\title{
Microwave Irradiation or Low Temperature Improved Synthesis of Antiparasitic Morita-Baylis-Hillman Adducts
}

\author{
Claudio G. L. Junior, Fábio P. L. Silva, Ramon G. de Oliveira, Fernanda L. Subrinho, \\ Natália G. de Andrade and Mário L. A. A. Vasconcellos* \\ Departamento de Química, Universidade Federal da Paraíba, Campus I, \\ João Pessoa-PB 58059-900, Brazil
}

\begin{abstract}
Relatamos a síntese de 24 adutos de Morita-Baylis-Hillman (3a-31/6a-6l) usando irradiação de microondas a $80{ }^{\circ} \mathrm{C}$ ou protocolo convencional a $0{ }^{\circ} \mathrm{C}$ para promover reações entre aldeídos aromáticos e acrilato de metila ou acrilonitrila (81-99\%). Provamos que a reação de formação do acrilato de 2-hidroxi(4-bromofenil)metila é reversível a $120{ }^{\circ} \mathrm{C}$.

It is reported the synthesis of 24 Morita-Baylis-Hillman adducts (3a-31/6a-61) using microwave irradiation at $80^{\circ} \mathrm{C}$ or conventional protocol at $0{ }^{\circ} \mathrm{C}$ to promote the reactions between aromatic aldehydes and methyl acrylate or acrylonitrile (81-99\%). It is shown that the reaction of 2-hydroxy(4-bromophenyl)methyl acrylate formation is reversible at $120^{\circ} \mathrm{C}$.
\end{abstract}

Keywords: microwave-promoted reactions, Morita-Baylis-Hillman reaction, thermodynamic control, temperature effect, antiparasitic compounds

\section{Introduction}

The Morita-Baylis-Hillman reaction (MBHR, Scheme 1) is one of the most powerful and versatile carbon-carbon bond forming methods in organic synthesis. ${ }^{1,2}$ An inconvenience associated with this reaction is the long reaction times, that last up to 65 days. ${ }^{2}$ Due to the synthetic utility of these MoritaBaylis-Hillman adducts (MBHA), several protocols have been described to improve the reaction time and yields, such as the ultrasound use,${ }^{3}$ high pressures,${ }^{4}$ ionic liquids use ${ }^{5}$ and several experimental protocol variations. ${ }^{2}$

Microwave heating is an ecofriendly approach and a valuable tool for synthetic chemists because it is possible to enhance the reaction rate and, in many cases, to improve product yields. ${ }^{6}$ There is an enormous growth of interest in this promising technique for promoting reactions.? Nevertheless, considering its significance, a reduced number of reports about the use of microwave irradiation promoting MBHR has been published. ${ }^{8}$

Despite the fact that MBHR has already more than 40 years of existence, ${ }^{1}$ the general mechanism of the reaction is still highlighted in discussions on the scientific community. The first catalytic cycle suggested by Hill and Isaacs ${ }^{9}$ is

*e-mail: mlaav@quimica.ufpb.br even accepted, but being the rate-determining step (RDS) remains at the center of the debate. Differently as proposed by Hill and Isaacs, McQuade and co-workers ${ }^{10}$ (nonprotic condition), Aggarwal (protic condition), ${ }^{11}$ supported by Amarante et al. ${ }^{12}$ (through electrospray ionization mass spectrometry (ESI-MS) experiments), consider the last step of this catalytic cycle as the RDS. However, these ways of thinking about this mechanism were recently expanded by Cantillo and Kappe ${ }^{13}$ in their unified mechanistic point of view. They proposed that the RDS can be changed, depending on the reagents, additives and experimental protocols of the reactions. ${ }^{13}$

In connection with our efforts towards the MBHR reactivity study, ${ }^{14}$ and aiming to expand the MBHA synthesis in one easily prepared step as an effective class of antiparasitic compounds, ${ }^{15}$ the present work show results about microwave irradiation promoting MBHR, the limitations found and, in some cases, the importance to carried out reactions at lower temperature. ${ }^{13}$

\section{Results and Discussion}

Initially, Table 1 is presented with the reactions between some aromatics aldehydes (1) and acrylonitrile (2) in the preparation of 3a-31 MBHA ${ }^{5,8,14-17}$ (Scheme 1). 


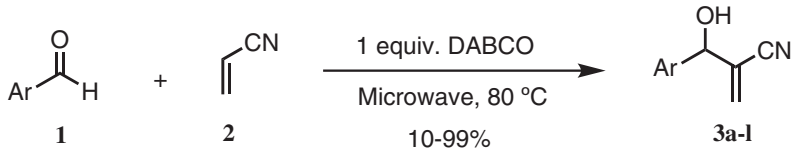

Scheme 1. Synthesis of MBHA 3a-3l (Table 1).

Fortunately, some of these adducts were obtained in excellent yields and in a very short reaction time (entries 2, 4-8, Table 1) at solvent-free condition. The adduct $3 \mathbf{c}$ (entry 3, Table 1) was obtained in $70 \%$ due to the more polar byproduct formation, which presented $[\mathrm{M}]^{+}=257 \mathrm{~m} / \mathrm{z}$ (detected by gas chromatography mass spectrometry (GC-MS) analysis) suggesting preparation of 4a (Figure 1), similar to that was noticed by Kundu et al. ${ }^{16}$ Aim to minimizing this byproduct, several microwave irradiation protocols were evaluated. Satisfactorily, the microwave irradiation at $80{ }^{\circ} \mathrm{C}$ during 5 min using THF as solvent was found as the optimized condition for this reaction (entry 4, Table 1). Differently, the corresponding $\mathbf{4 b}$ and $\mathbf{4 c}$ byproducts (Figure 1 ) were detected by GC-MS in very small proportion during the preparations of 3h-3i (entries 9-10, Table 1). However, a 4d byproduct (Scheme 1) appears once more in high amount on the preparation of $\mathbf{3 j}$ (entries 11, Table 1). The use of ethanol as solvent in the preparation of $\mathbf{3} \mathbf{j}$ increased slightly the

Table 1. Synthesis of MBHA 3a-3l using Microwave (Scheme 1) ${ }^{\mathrm{a}}$

\begin{tabular}{|c|c|c|c|c|}
\hline entry & $\mathrm{Ar}$ & MBHA & $\begin{array}{c}\text { Reaction time } \\
/ \mathrm{min}\end{array}$ & Yield / \% \\
\hline 1 & $\mathrm{C}_{6} \mathrm{H}_{5}$ & 3a & 60 & $86^{c}$ \\
\hline 2 & $o-\left(\mathrm{NO}_{2}\right) \mathrm{C}_{6} \mathrm{H}_{4}$ & $3 \mathbf{b}$ & 1.5 & $99^{\mathrm{b}}$ \\
\hline 3 & $m-\left(\mathrm{NO}_{2}\right) \mathrm{C}_{6} \mathrm{H}_{4}$ & $3 c$ & 1.5 & $70^{\mathrm{b}}$ \\
\hline 4 & $m-\left(\mathrm{NO}_{2}\right) \mathrm{C}_{6} \mathrm{H}_{4}$ & $3 c$ & 5 & $98^{\mathrm{b}, \mathrm{d}}$ \\
\hline 5 & $p-\left(\mathrm{NO}_{2}\right) \mathrm{C}_{6} \mathrm{H}_{4}$ & 3d & 1.5 & $99^{\mathrm{b}}$ \\
\hline 6 & $2-\mathrm{C}_{5} \mathrm{H}_{4} \mathrm{~N}$ & $3 e$ & 1.5 & $99^{\mathrm{b}}$ \\
\hline 7 & $3-\mathrm{C}_{5} \mathrm{H}_{4} \mathrm{~N}$ & $3 f$ & 1.5 & $99^{\mathrm{b}}$ \\
\hline 8 & $4-\mathrm{C}_{5} \mathrm{H}_{4} \mathrm{~N}$ & $3 g$ & 1.5 & $99^{\mathrm{b}}$ \\
\hline 9 & $p-(\mathrm{F}) \mathrm{C}_{6} \mathrm{H}_{4}$ & $3 h$ & 120 & $82^{\mathrm{c}, \mathrm{e}}$ \\
\hline 10 & $p-(\mathrm{Cl}) \mathrm{C}_{6} \mathrm{H}_{4}$ & $3 \mathbf{i}$ & 120 & $84^{c, g}$ \\
\hline 11 & $p-(\mathrm{Br}) \mathrm{C}_{6} \mathrm{H}_{4}$ & $3 \mathbf{j}$ & 30 & $54^{\mathrm{b}, \mathrm{fi}}$ \\
\hline 12 & $p-(\mathrm{Br}) \mathrm{C}_{6} \mathrm{H}_{4}$ & $3 \mathbf{j}$ & 60 & $63^{\mathrm{b}, \mathrm{g}, \mathrm{i}}$ \\
\hline 13 & 2- $\mathrm{C}_{10} \mathrm{H}_{7}$ & $3 k$ & 120 & $10^{\mathrm{c}, \mathrm{h,i}}$ \\
\hline 14 & $3,4-\left(\mathrm{OCH}_{2} \mathrm{O}\right) \mathrm{C}_{6} \mathrm{H}_{3}$ & 31 & 120 & $\operatorname{trace}^{\mathrm{e}, \mathrm{i}}$ \\
\hline
\end{tabular}

a Reactions were carried out using aldehyde $(0.5 \mathrm{mmol})$, acrylonitrile (3.1 mmol) and $\mathrm{DABCO}(0.5 \mathrm{mmol})$ at $80{ }^{\circ} \mathrm{C}$ under microwave irradiation; bisolated yield; ${ }^{\mathrm{c}}$ conversion determined by GC-MS; ${ }^{\mathrm{d}} 0.5 \mathrm{~mL}$ of THF were used as solvent; ${ }^{e} 0.5 \mathrm{~mL}$ of methanol were used as solvent; ${ }^{\mathrm{f}}>20 \%$ of $\mathbf{4 d}$ was detected by GC-MS; ${ }^{\mathrm{g}} 0.5 \mathrm{~mL}$ of ethanol were used as solvent; ${ }^{\mathrm{h}} 0.5 \mathrm{~mL}$ de DMSO were used as solvent; i others solvents were evaluated, but without success. yield (entry 12). However, reactions on preparation of $\mathbf{3 j}$ at 100 and $120^{\circ} \mathrm{C}$ lead to lower yields.

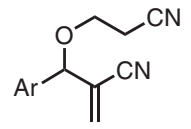

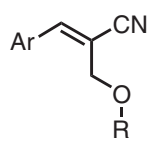

Figure 1. Detected byproducts by GC-MS analysis: $4 \mathbf{a} \mathrm{Ar}=m-\left(\mathrm{NO}_{2}\right) \mathrm{C}_{6} \mathrm{H}_{4}$; 4b $\mathrm{Ar}=p-(\mathrm{F}) \mathrm{C}_{6} \mathrm{H}_{4} ; \mathbf{4 c} \mathrm{Ar}=p-(\mathrm{Cl}) \mathrm{C}_{6} \mathrm{H}_{4} ; 4 \mathbf{d} \mathrm{Ar}=p-(\mathrm{Br}) \mathrm{C}_{6} \mathrm{H}_{4}$; 4e $\mathrm{Ar}=2-\mathrm{C}_{10} \mathrm{H}_{7} ; \mathbf{5 a} 2-\mathrm{C}_{10} \mathrm{H}_{7} ; \mathbf{5 b} 3,4-\left(\mathrm{OCH}_{2} \mathrm{O}\right) \mathrm{C}_{6} \mathrm{H}_{3} ; \mathrm{R}=\mathrm{CH}_{3}, \mathrm{CH}_{3} \mathrm{CH}_{2}$.

The synthesis of $\mathbf{3 k}$ occurs in very low yield even after uninterrupted $2 \mathrm{~h}$ of microwave irradiation, in several solvents (entry 13, Table 1). Moreover, preparation of $\mathbf{3 1}$ did not occur even after a continuous $1 \mathrm{~h}$ irradiation (entry 14, Table 1). The corresponding $\mathbf{4 e}$ and $\mathbf{5 a}$ byproducts were also detected by GC-MS (Figure 1). The origin of $\mathbf{5 a}$ is probably due to the nucleophilic Michael addition of alcohol on the activated double bounds followed by isomerization.

In 1997, Leahy and Rafel ${ }^{17}$ have reported an unexpected rate accelerations when they performed the MBHR at $0{ }^{\circ} \mathrm{C}$. They have rationalized this in terms of the greater stability at $0{ }^{\circ} \mathrm{C}$ of the $Z$-aza-enolate transition state (TS) as compared to the $E$-TS. ${ }^{17}$ After that, this no common temperature effect on the exquisite $\mathrm{MBH}$ reaction were also described by our group ${ }^{18}$ and by Porto et al. ${ }^{19}$ More recently, our group presented an alternative explanation to this temperature effect, by suggesting that the entropic term $(-T \Delta S>0)$ could be pivotal on the rate of the Morita-Baylis-Hillman reaction at $0{ }^{\circ} \mathrm{C} .{ }^{20}$ In the theoretical and experimental results described by Cantillo and Kappe,,$^{13}$ this proposal was confirmed. These experiments carried out by Cantillo and $\mathrm{Kappe}^{13}$ also proved that the reaction between benzaldehyde and methyl acrylate catalyzed by $\mathrm{DABCO}$ in methanol is reversible at $120{ }^{\circ} \mathrm{C} .{ }^{13}$ Aiming to prove the equilibrium formation in our reactions, our group performed a similar experiment carried out by Cantillo and Kappe, ${ }^{13}$ where the pure adduct $\mathbf{6 j}$ (Scheme 2) was dissolved in $1 \mathrm{~mL}$ of methanol in the presence of DABCO (2 equiv.) and heated under microwave irradiation at $120{ }^{\circ} \mathrm{C}$ for $2 \mathrm{~h}$. After that, it was observed the $p$-bromobenzaldehyde and methyl acrylate formation in considerable amount (ca. 55\% by GC-MS). After this irradiation, the reaction mixture was carried out at $0{ }^{\circ} \mathrm{C}$ under magnetic stirring for $24 \mathrm{~h}$ and the equilibrium shifted once again to the formation of methyl 2-[hydroxy(4-bromophenyl)methyl]acrylate (6j) confirming as expected, the reversible nature for this sophisticated reaction (Scheme 2).

Based on these facts it was performed the synthesis of 3a, $\mathbf{3 h}, \mathbf{3 i}$ and $\mathbf{3 l}$ adducts at lower temperature $\left(0{ }^{\circ} \mathrm{C}\right)$. The results are presented in Table 2. It is possible to highlight 


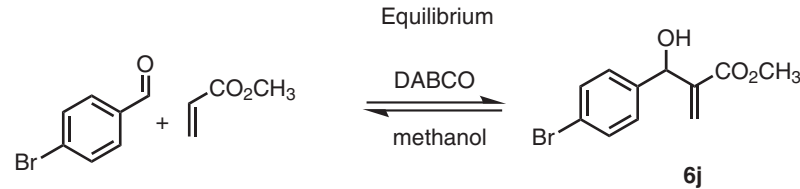

Scheme 2. The reversible character of MBH reaction between $p$-bromobenzaldehyde, methyl acrylate, and methyl 2-[hydroxy(4bromophenyl)methyl]acrylate (6j) on protic solvent.

in Table 2 that all reactions at $0{ }^{\circ} \mathrm{C}$ occur in excellent to quantitative yields (90-99\%, entries 1-12, Table 2). Interestingly, the adduct $3 \mathbf{k}$ was obtained successfully using this method, without forming any byproduct (entry 11). Moreover, the most interesting, this MBHA 31 was prepared in high yields at low temperature protocol using methanol as solvent (compare entry 14 (Table 1) vs. entry 12 (Table2)). Solvent-free condition is not efficient in this case.

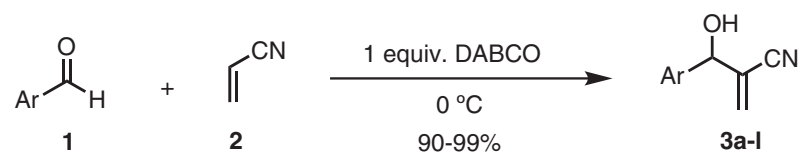

Scheme 3. Synthesis of MBHA 3a-3l (Table 2).

Table 2. Synthesis of MBHA 3a-3l at low temperature (Scheme 3) ${ }^{\mathrm{a}}$

\begin{tabular}{lcccc}
\hline entry & $\mathrm{Ar}$ & MBHA & Reaction time & Yield $/ \%$ \\
\hline 1 & $\mathrm{C}_{6} \mathrm{H}_{5}$ & $\mathbf{3 a}$ & $20 \mathrm{~h}$ & 99 \\
2 & $o-\left(\mathrm{NO}_{2}\right) \mathrm{C}_{6} \mathrm{H}_{4}$ & $\mathbf{3 b}$ & $40 \mathrm{~min}$ & 99 \\
3 & $m-\left(\mathrm{NO}_{2}\right) \mathrm{C}_{6} \mathrm{H}_{4}$ & $\mathbf{3 c}$ & $25 \mathrm{~min}$ & 99 \\
4 & $p-\left(\mathrm{NO}_{2}\right) \mathrm{C}_{6} \mathrm{H}_{4}$ & $\mathbf{3 d}$ & $15 \mathrm{~min}$ & 99 \\
5 & $2-\mathrm{C}_{5} \mathrm{H}_{4} \mathrm{~N}$ & $\mathbf{3 e}$ & $30 \mathrm{~min}$ & 99 \\
6 & $3-\mathrm{C}_{5} \mathrm{H}_{4} \mathrm{~N}$ & $\mathbf{3 f}$ & $50 \mathrm{~min}$ & 99 \\
7 & $4-\mathrm{C}_{5} \mathrm{H}_{4} \mathrm{~N}$ & $\mathbf{3 g}$ & $10 \mathrm{~min}$ & 99 \\
8 & $p-(\mathrm{F}) \mathrm{C}_{6} \mathrm{H}_{4}$ & $\mathbf{3 h}$ & $20 \mathrm{~h}$ & 95 \\
9 & $p-\left(\mathrm{Cl}_{2} \mathrm{C}_{6} \mathrm{H}_{4}\right.$ & $\mathbf{3 i}$ & $3.5 \mathrm{~h}$ & 99 \\
10 & $p-\left(\mathrm{Br}_{6} \mathrm{C}_{6} \mathrm{H}_{4}\right.$ & $\mathbf{3 j}$ & $4 \mathrm{~h}$ & 98 \\
11 & $2-\mathrm{C}_{10} \mathrm{H}_{7}$ & $\mathbf{3 k}$ & $10 \mathrm{~h}$ & 98 \\
12 & $3,4-\left(\mathrm{OCH}_{2} \mathrm{O}\right) \mathrm{C}_{6} \mathrm{H}_{3}$ & $\mathbf{3 l}$ & $3 \mathrm{days}$ & $90^{c}$ \\
\hline
\end{tabular}

${ }^{a}$ Reactions were carried out using aldehyde $(0.5 \mathrm{mmol})$, acrylonitrile $(3.1 \mathrm{mmol})$ and $\mathrm{DABCO}(0.5 \mathrm{mmol})$ at $0^{\circ} \mathrm{C}$ under conventional protocol; bisolated yield; ${ }^{\mathrm{c}} 0.5 \mathrm{~mL}$ of methanol was used as solvent.

Subsequently, our group investigated the reaction between aromatic aldehydes $(0.5 \mathrm{mmol})$ and methyl acrylate (7) (10.6 equiv.) promoted by DABCO $(0.5 \mathrm{mmol})$ on the preparations of $\mathbf{6 a - 6 1} .^{5,8,14,15,20,21}$ The reactions were microwave-promoted at $80^{\circ} \mathrm{C}$ and the results are presented in Table 3 (Scheme 4).

It was observed that adducts $6 \mathbf{b}-\mathbf{6 g}$ were obtained in quantitative yield and short reaction times (entries 2-7, Table 3). However, the adducts $\mathbf{6 a}, \mathbf{6 h}, \mathbf{6 i}, \mathbf{6 j}, \mathbf{6 k}$ and $\mathbf{6}$

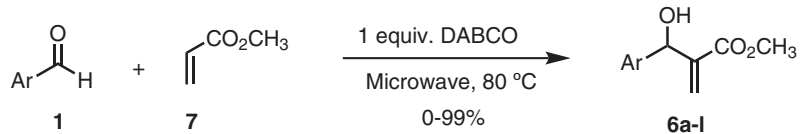

Scheme 4. Microwave-promoted synthesis of MBHA 6a-6l.

Table 3. Microwave irradiation on the synthesis of MBH adducts $\mathbf{6 a - 6 1}$

\begin{tabular}{lcccc}
\hline entry & $\mathrm{Ar}$ & MBHA & $\begin{array}{c}\text { Reaction } \\
\text { time / min }\end{array}$ & Yield $\mathrm{a,b} / \%$ \\
\hline 1 & $\mathrm{C}_{6} \mathrm{H}_{5}$ & $\mathbf{6 a}$ & 120 & $\mathrm{NR}$ \\
2 & $o-\left(\mathrm{NO}_{2}\right) \mathrm{C}_{6} \mathrm{H}_{4}$ & $\mathbf{6 b}$ & 10 & 99 \\
3 & $m-\left(\mathrm{NO}_{2}\right) \mathrm{C}_{6} \mathrm{H}_{4}$ & $\mathbf{6 c}$ & 5 & 99 \\
4 & $p-\left(\mathrm{NO}_{2}\right) \mathrm{C}_{6} \mathrm{H}_{4}$ & $\mathbf{6 d}$ & 5 & 99 \\
5 & $2-\mathrm{C}_{5} \mathrm{H}_{4} \mathrm{~N}$ & $\mathbf{6 e}$ & 10 & 99 \\
6 & $3-\mathrm{C}_{5} \mathrm{H}_{4} \mathrm{~N}$ & $\mathbf{6 f}$ & 10 & 99 \\
7 & $4-\mathrm{C}_{5} \mathrm{H}_{4} \mathrm{~N}$ & $\mathbf{6 g}$ & 5 & 99 \\
8 & $p-(\mathrm{F}) \mathrm{C}_{6} \mathrm{H}_{4}$ & $\mathbf{6 h}$ & 120 & $\mathrm{NR}$ \\
9 & $p-\left(\mathrm{Cl}_{2}\right) \mathrm{C}_{6} \mathrm{H}_{4}$ & $\mathbf{6 i}$ & 120 & $\mathrm{NR}$ \\
10 & $p-\left(\mathrm{Br}_{2} \mathrm{C}_{6} \mathrm{H}_{4}\right.$ & $\mathbf{6 j}$ & 120 & $\mathrm{NR}$ \\
11 & $2-\mathrm{C}_{10} \mathrm{H}_{7}$ & $\mathbf{6 k}$ & 120 & $\mathrm{NR}$ \\
12 & $3,4-\left(\mathrm{OCH}_{2} \mathrm{O}\right) \mathrm{C}_{6} \mathrm{H}_{3}$ & $\mathbf{6}$ & 120 & $\mathrm{NR}$ \\
\hline
\end{tabular}

a Reactions were carried out using aldehyde $(0.5 \mathrm{mmol})$, methyl acrylate $(5.3 \mathrm{mmol})$ and $\mathrm{DABCO}(0.5 \mathrm{mmol})$ at $80^{\circ} \mathrm{C}$ under microwave irradiation; bisolated yield; $\mathrm{NR}=$ no reaction.

were not formed $2 \mathrm{~h}$ of continuum microwave irradiation (entries 1, 8-12, Table 3). It was also investigated other protic and noprotic solvents such as methanol, ethanol, DMF, THF and DMSO, but the results were not satisfactory. The temperature increase did not improved yields, which may be caused by DABCO degradation at temperatures greater than $90{ }^{\circ} \mathrm{C}$ or the possible equilibrium formation when carried out at high temperature, as demonstrated in the Scheme 2.

The studies developed by Cantillo and Kappe ${ }^{13}$ pointed the influence of temperature on constant equilibrium for the reaction in preparation of adducts $\mathbf{6 a}$ and $\mathbf{6 d}$. They reported ca. $330 \mathrm{~K}\left(57^{\circ} \mathrm{C}\right)$ to prepare $6 \mathbf{a}$ becomes to be endergonic, explaining the fact that this reaction occur in only moderated yield at elevated temperatures (entry 1, Table 4). On the other hand, this fact also fully explains our failure in the reaction using microwave irradiation at $80^{\circ} \mathrm{C}$ for preparation of $\mathbf{6 a}$ and differently, the success in using low temperature. The similar calculations indicated that the preparation of $\mathbf{6 d}$ requires a higher temperature to be endergonic $\left(c a .107^{\circ} \mathrm{C}\right) .{ }^{13}$ Then, the excellent yields obtained in this report, when the $\mathrm{NO}_{2}$ moiety or the 2, 3 or 4-pyridine is present in the aldehyde are understandable now.

Finally, it is presented in Table 4 (Scheme 5), our results aimed to optimize the $\mathbf{6 a}, \mathbf{6 h}-\mathbf{6 1}$ MBHA yields using protocols at low temperature. 
Table 4. Synthesis of MBHA $\mathbf{6 a - 6 1}$ at low temperature

\begin{tabular}{lcccc}
\hline entry & $\mathrm{R}$ & MBHA & Reaction time & Yield $^{\mathrm{b}} \%$ \\
\hline 1 & $\mathrm{C}_{6} \mathrm{H}_{5}$ & $\mathbf{6 a}$ & $48 \mathrm{~h}$ & $99^{\mathrm{c}}$ \\
2 & $o-\left(\mathrm{NO}_{2}\right) \mathrm{C}_{6} \mathrm{H}_{4}$ & $\mathbf{6 b}$ & $3 \mathrm{~h}$ & 92 \\
3 & $m-\left(\mathrm{NO}_{2}\right) \mathrm{C}_{6} \mathrm{H}_{4}$ & $\mathbf{6 c}$ & $2 \mathrm{~h}$ & 98 \\
4 & $p-\left(\mathrm{NO}_{2}\right) \mathrm{C}_{6} \mathrm{H}_{4}$ & $\mathbf{6 d}$ & $3 \mathrm{~h}$ & 85 \\
5 & $2-\mathrm{C}_{5} \mathrm{H}_{4} \mathrm{~N}$ & $\mathbf{6 e}$ & $5 \mathrm{~h}$ & 95 \\
6 & $3-\mathrm{C}_{5} \mathrm{H}_{4} \mathrm{~N}$ & $\mathbf{6 f}$ & $2 \mathrm{~h}$ & 98 \\
7 & $4-\mathrm{C}_{5} \mathrm{H}_{4} \mathrm{~N}$ & $\mathbf{6 g}$ & $2 \mathrm{~h}$ & 81 \\
8 & $p-\left(\mathrm{F}_{6} \mathrm{C}_{6} \mathrm{H}_{4}\right.$ & $\mathbf{6 h}$ & $48 \mathrm{~h}$ & $99^{c}$ \\
9 & $p-\left(\mathrm{Cl}_{6} \mathrm{C}_{6} \mathrm{H}_{4}\right.$ & $\mathbf{6 i}$ & $48 \mathrm{~h}$ & $95^{\mathrm{c}}$ \\
10 & $p-\left(\mathrm{Br}_{2} \mathrm{C}_{6} \mathrm{H}_{4}\right.$ & $\mathbf{6 j}$ & $72 \mathrm{~h}$ & $90^{\mathrm{c}}$ \\
11 & $2-\mathrm{C}_{10} \mathrm{H}_{7}$ & $\mathbf{6 k}$ & $16 \mathrm{days}$ & 85 \\
12 & $3,4-\left(\mathrm{OCH}_{2} \mathrm{O}\right) \mathrm{C}_{6} \mathrm{H}_{3}$ & $\mathbf{6 l}$ & 9 days & $63^{c}$ \\
13 & $3,4-\left(\mathrm{OCH}_{2} \mathrm{O}\right) \mathrm{C}_{6} \mathrm{H}_{3}$ & $\mathbf{6 l}$ & $18 \mathrm{~h}$ & $90^{\mathrm{d}}$ \\
\hline
\end{tabular}

${ }^{\mathrm{a}}$ Reactions were carried out using aldehyde $(0.5 \mathrm{mmol})$, methyl acrylate $(5.3 \mathrm{mmol})$ and $\mathrm{DABCO}(0.5 \mathrm{mmol})$ at $0^{\circ} \mathrm{C}$ under conventional protocol; bisolated yield; ${ }^{\mathrm{c}} 0.5 \mathrm{~mL}$ of methanol were used as solvent; ${ }^{\mathrm{d}} 5 \mathrm{~mL}$ of DMF: $\mathrm{H}_{2} \mathrm{O}(9: 1)$ were used as solvent.

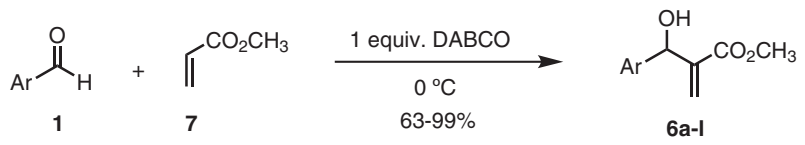

Scheme 5. Synthesis of MBHA 6a-6l at low temperature (Table 4).

In fact, this low temperature protocol was very efficient for the synthesis of adducts $\mathbf{6 a}$ and $\mathbf{6 h}-\mathbf{6} \mathbf{l}$ which were formed in high or quantitative yields. Our group discovered that the moderated yield obtained using methanol as solvent to prepare MBHA $6 \mathbf{1}$ (entry 12, Table 4, 63\%) could be significantly improved by changing of the solvent and concentration of this reaction (entry 13, Table 4). This work was also performed using 0.8/0.6/0.4/0.2 equiv. of $\mathrm{DABCO}$ as promoter. However, no efficient results were obtained. All experiments in this report were also carried out at room temperature. However, the results were far below those which were made at low temperatures or under microwave irradiation.

\section{Conclusions}

In conclusion, it is reported that the microwavepromoted MBHR at $80^{\circ} \mathrm{C}$ was efficient with acrylonitrile or methyl acrylate in the more reactive aromatic aldehydes (e.g. $p$-nitrobenzaldehyde), resulting the high yields and very short reaction times. However, microwave irradiation MBHR at $80{ }^{\circ} \mathrm{C}$ with acrylonitrile or methyl acrylate in the less reactive aromatic aldehydes (e.g. benzaldehyde) was inefficient. In these cases, reactions carried out at
$0{ }^{\circ} \mathrm{C}$ were much more efficient, by eliminating equilibria of reactions, minimizing byproducts and thus, generating excellent MBHA yields. Moreover, it was proved in the present work the reversible character of the preparation at high temperature of methyl 2-[hydroxy(4-bromophenyl) methyl] acrylate. Thus, these results were rationalized considering the possibility of thermodynamic control in MBHR. ${ }^{13}$ In summary, it was synthesized 24 MBHA in high or quantitative yields (81-99\%) by changing the experimental protocols.

\section{Supplementary Information}

Experimental details and spectroscopic data are available free of charge at http://jbcs.sbq.org.br as PDF file.

\section{Acknowledgments}

The authors gratefully acknowledge Conselho Nacional de Desenvolvimento Científico e Tecnológico (CNPq), Coordenação de Aperfeiçoamento de Pessoal de Nível Superior (CAPES) and Fundação de Apoio a Pesquisa do Estado da Paraíba (FAPESQ-PB) for financial support.

\section{References}

1. Morita, K.; Suzuki, Z.; Hirose, H.; Bull. Chem. Soc. Jpn. 1968, 41, 2815; Baylis, A. B.; Hillman, M. E. D.; German Patent 2155113 1972. (CA 77:34174q)

2. For reviews, see: Basavaiah, D.; Reddy, B. S.; Badsara, S. S.; Chem. Rev. 2010, 110, 5447; Basavaiah, D.; Rao, K. V.; Reddy, R.; J. Chem. Soc. Rev. 2007, 36, 1581; Masson, G.; Housseman, C.; Zhu, J.; Angew. Chem., Int. Ed. 2007, 46, 4614; Basavaiah, D.; Rao, J. A.; Satyanarayana, T.; Chem. Rev. 2003, 103, 811; Langer, P.; Angew. Chem., Int. Ed. 2000, 39, 3049; Basavaiah, D.; Rao, P. D.; Hyma, R. S.; Tetrahedron 1996, 52, 8001; Drewes, S. E.; Roos, G. H. P.; Tetrahedron 1988, 44, 4653.

3. Coelho, F.; Almeida, W. P.; Veronese, D.; Mateus, C. R.; Lopes, E. C. S.; Rossi, R. C.; Silveira, G. P. C.; Pavam, C. H.; Tetrahedron 2002, 58, 7437.

4. Hill, J. S.; Isaacs, N. S.; J. Chem. Res., Synop. 1988, 330.

5. de Souza, R. O. M. A.; Fregadolli, P. H.; Aguiar, L. C.; Gonçalves, K. M.; Pereira, V. L. P.; Filho, L. C.; Esteves, P. M.; Vasconcellos, M. L. A. A.; Antunes, O. A. C.; Lett. Org. Chem. 2006, 3, 936 .

6. Herrero, M. A.; Kremsner, J. M.; Kappe, C. O.; J. Org. Chem. 2008, 73, 36; Loupy, A.; Microwaves in Organic Synthesis; Wiley: Weinheim, 2006.

7. Leadbeater, N. E.; Arkivoc 2011, 5, 127; Neves Filho, R. A. W.; Oliveira, R. N.; Srivastava, R. M.; J. Braz. Chem. Soc. 2007, 18, 1410; Barreto, A. F. S.; Vercillo, O. E.; Andrade, 
C. K. Z.; J. Braz. Chem. Soc. 2011, 22, 462; Lenardão, E. J.; Trecha, D. O.; Ferreira, P. C.; Jacob, R. G.; Perin, G.; J. Braz. Chem. Soc. 2009, 20, 93; Reddy, L. V.; Suman, A.; Beevi, S. S.; Mangamoori, L. N.; Mukkanti, K.; Pal, S.; J. Braz. Chem. Soc. 2010, 21, 98; Silva, A. R.; Silva, A. M.; Ferreira, A. B. B.; Bernardes, B. O.; Costa, R. L.; J. Braz. Chem. Soc. 2008, 19, 1230.

8. Kundu, M. K.; Mukherjee, S. B.; Balu, N.; Padmakumar, R.; Bhat, S. V.; Synlett 1994, 444.

9. Hill, J. S.; Isaacs, N. S.; J. Phys. Org. Chem. 1990, 3, 285; Hill, J. S.; Isaacs, N. S.; J. Chem. Res. 1988, 330.; Hill, J. S.; Isaacs, N. S.; Tetrahedron Lett. 1996, 27, 5007.

10. Price, K. E.; Broadwater, S. J.; Jung, H. M.; McQuade, D. T.; Org. Lett. 2005, 7, 147; Price, K. E.; Broadwater, S. J.; Walker, B. J.; McQuade, D. T.; J. Org. Chem. 2005, 70, 3980.

11. Aggarwal, V. K.; Fulford, S. Y.; Lloyd-Jones, G. C.; Angew. Chem., Int. Ed. 2005, 44, 1706.

12. Amarante, G. W.; Milagre, H. M. S.; Vaz, B. G.; Ferreira, B. R. V.; Eberlin, M. N.; Coelho, F.; J. Org. Chem. 2009, 74, 3031.

13. Cantillo, D.; Kappe, C. O.; J. Org. Chem. 2010, 75, 8615.

14. de Souza, R. O. M. A.; Pereira, V. L. P.; Esteves, P. M.; Vasconcellos, M. L. A. A.; Tetrahedron Lett. 2008, 49, 5902; de Souza, R. O. M. A.; Souza, A. L. F.; Fernandez, T. L.; Silva, A. C.; Pereira, V. L. P.; Esteves, P. M.; Vasconcellos, M. L. A. A.; Antunes, O. A. C.; Lett. Org. Chem. 2008, 5, 379; de Souza, R. O. M. A.; Meireles, B. A.; Aguiar, C. S.; Vasconcellos, M. L. A. A.; Synthesis 2004, 1595.

15. Vasconcellos, M. L. A. A.; Silva, T. M. S.; Camara, C. A.; Martins, R. M.; Lacerda, K. M.; Lopes, H. M.; Pereira, V. L. P.; de Souza, R. O. M. A.; Crespo, L. T. C.; Pest Manag. Sci. 2006, 62, 288; de Souza, R. O. M. A.; Pereira, V. L. P.; Muzitano,
M. F.; Rossi-Bergmann, B.; Filho, E. B. A.; Vasconcellos, M. L. A. A.; Eur. J. Med. Chem. 2007, 42, 99; Barbosa, T. C.; Junior, C. G. L.; Silva, F. P. L.; Lopes, H. M.; Figueiredo, L. R. F.; Souza, S. C. O.; Batista, G. N.; Silva, T. G.; Silva, T. M.; Oliveira, M. R.; Vasconcellos, M. L. A. A.; Eur. J. Med. Chem. 2009, 44, 1726; Sandes, J. M.; Borges, A. R.; Junior, C. G. L.; Silva, F. P. L.; Carvalho, G. A. U.; Rocha, G. B.; Vasconcellos, M. L. A. A.; Figueiredo, R. C. B. Q.; Bioorg. Chem. 2010, 38, 190; Barbosa, T. P.; Sousa, S. C. O.; Amorim, F. M.; Rodrigues,Y. K. S.; de Assis, P. A.C.; Caldas, J. P. A.; Oliveira, M. R.; Vasconcellos, M. L. A. A.; Bioorg. Med. Chem. 2011, 19, 4250; Silva, F. P. L.; de Assis, P. A.C.; Junior, C. G. L.; de Andrade, N. G.; da Cunha, S. M..D.; Oliveira, M. R.; Vasconcellos, M. L. A. A.; Eur. J. Med. Chem. 2011, 46, 4295. doi:10.1016/j.ejmech.2011.06.036.

16. Kundu, M. K.; Sundar, N.; Kumar, S. K.; Bhat, S. V.; Biswas, S.; Valecha, N.; Bioorg. Med. Chem. Lett. 1999, 9, 731.

17. Rafel, S.; Leahy, S. W.; J. Org. Chem. 1997, 62, 1521.

18. de Souza, R. O. M. A.; Vasconcellos, M. L. A. A.; Synth. Commun. 2003, 33, 1393; de Souza, R. O. M. A.; Vasconcellos, M. L. A. A.; Catal. Commun. 2004, 5, 21.

19. Porto, R. S.; Amarante, G. W.; Cavallaro, M.; Poppi, R. J.; Coelho, F.; Tetrahedron Lett. 2009, 50, 1184.

20. Junior, C. G. L.; de Assis, P. A. C.; Silva, F. P. L.; Sousa, S. C. O.; Andrade, N. G.; Barbosa, T. P.; Nerís, P. L. N.; Segundo, L. V. G.; Anjos, I. C.; Carvalho, G A. U.; Rocha, G. B.; Oliveira, M. R.; Vasconcellos, M. L. A. A.; Bioorg. Chem. 2010, 38, 279.

21. Jeong, Y.; Ryu, J. S.; J. Org. Chem. 2010, 75, 4183.

Submitted: March 18, 2011 Published online: August 25, 2011 


\title{
Microwave Irradiation or Low Temperature Improved Synthesis of Antiparasitic Morita-Baylis-Hillman Adducts
}

\author{
Claudio G. L. Junior, Fábio P. L. Silva, Ramon G. de Oliveira, Fernanda L. Subrinho, \\ Natália G. de Andrade and Mário L. A. A. Vasconcellos* \\ Departamento de Química, Universidade Federal da Paraíba, Campus I, \\ João Pessoa-PB 58059-900, Brazil
}

\section{Experimental}

\section{General}

All commercially available reagents and solvent were obtained from the commercial providers and used without further purification. Reactions were monitored by TLC (thin layer chromatography) using Silica gel 60 UV254 Macherey-Nagel pre-coated silica gel plates; detection was by means of a UV lamp. Flash column chromatography was performed on 300-400 mesh silica gel. Organic layers were dried over anhydrous $\mathrm{MgSO}_{4}$ or $\mathrm{Na}_{2} \mathrm{SO}_{4}$ prior to evaporation on a rotary evaporator. Reactions requiring microwave irradiation were performed in a microwave reactor model system Discover benchmate with temperature monitored by built-in infrared sensor. ${ }^{1} \mathrm{H}$ and ${ }^{13} \mathrm{C}$ nuclear magnetic resonance (NMR) spectra were recorded using Varian Mercury Spectra AC 20 spectrometer $\left(200 \mathrm{MHz}\right.$ for ${ }^{1} \mathrm{H}, 50 \mathrm{MHz}$ for $\left.{ }^{13} \mathrm{C}\right)$. Chemical shifts were reported relative to internal tetramethylsilane $(\delta 0.00 \mathrm{ppm})$ for ${ }^{1} \mathrm{H}$, and $\mathrm{CDCl}_{3}(\delta 77.0 \mathrm{ppm})$ for ${ }^{13} \mathrm{C}$. Fourier transform infrared (FT-IR) spectra were recorded on a Shimadzu spectrophotometer model IRPrestige-21 in KBr pellets. Mass spectrometric analysis was performed on gas chromatography mass spectrometry (GC-MS) analysis (SHIMADZU GCMS-QP2010). The not new Morita-Baylis-Hillman adducts (MBHA) prepared in this report were characterized by ${ }^{1} \mathrm{H},{ }^{13} \mathrm{C}$ NMR and FT-IR in accordance with the described physical data.

General procedure for the microwave-assisted synthesis of $M B H A$

The corresponding aldehydes $(0.5 \mathrm{mmol})$, acrylonitrile $(0.2 \mathrm{~mL}, 3.1 \mathrm{mmol})$ or methyl acrylate $(0.5 \mathrm{~mL}, 5.3 \mathrm{mmol})$, $0.5 \mathrm{~mL}$ of solvent (see Tables) and DABCO (56 mg, $1 \mathrm{mmol}$ ) were placed in a $10 \mathrm{~mL}$ glass microwave tube with magnetic

*e-mail: mlaav@quimica.ufpb.br stirrer at $80^{\circ} \mathrm{C}$ (temperature monitored by built-in infrared sensor) for 1.5-120 $\mathrm{min}$. After the complete reaction, the mixture was brought to room temperature and was directly filtered through silica gel, using hexane/ethyl acetate (7:3) as solvent and the reaction products were concentrated under reduced pressure.

General procedure for the synthesis of MBHA at low temperature

Reactions were carried out using the corresponding aldehydes $(0.5 \mathrm{mmol})$, acrylonitrile $(0.2 \mathrm{~mL}, 3.1 \mathrm{mmol})$ or methyl acrylate $(0.5 \mathrm{~mL}, 5.3 \mathrm{mmol}), 0.5 \mathrm{~mL}$ of solvent (see tables) and DABCO (56 mg, $0.5 \mathrm{mmol}$ ) at $0{ }^{\circ} \mathrm{C}$ under stirrer for the time indicated in Tables 2 and 4 . After that, the reaction media was directly filtered through silica gel, using hexane/ethyl acetate (7:3) as solvent and the reaction products were concentrated under reduced pressure.

2-[(Hydroxyphenyl)methyl] acrylonitrile (3a)

IR (KBr) $v_{\max } / \mathrm{cm}^{-1} 3444,2229 ;{ }^{1} \mathrm{H}$ NMR (200 MHz, $\left.\mathrm{CDCl}_{3}\right) \delta 7.42-7.37(\mathrm{~m}, 5 \mathrm{H}), 6.13(\mathrm{~d}, 1 \mathrm{H}, J 1.6 \mathrm{~Hz}), 6.06$ (d, 1H, J $1.6 \mathrm{~Hz}), 5.33$ (m, 1H), 2.24 (d, 1H, J 4.0 Hz); ${ }^{13} \mathrm{C} \mathrm{NMR}\left(50 \mathrm{MHz}, \mathrm{CDCl}_{3}\right) \delta 139.3,130.1,129.1,129.1$, 126.7, 126.3, 117.1, 74.2.

\section{2-[Hydroxy(2-nitrophenyl)methyl] acrylonitrile (3b)}

IR (KBr) $v_{\max } / \mathrm{cm}^{-1} 3345,2228,1348,1609,1520$; ${ }^{1} \mathrm{H}$ NMR $\left(200 \mathrm{MHz}, \mathrm{CDCl}_{3}\right) \delta 8.01(\mathrm{dd}, 1 \mathrm{H}, J 8.0 / 1.4 \mathrm{~Hz})$, 7.84 (dd, 1H, J 6.0/1.8), 7.72 (ddd, 1H, J 8.0/1.8/1.4 Hz), 7.52 (ddd, 1H, J 8.0/1.6/1.4 Hz), 6.12 (d, 1H, J $1.4 \mathrm{~Hz}$ ), 6.09 (d, 1H, J 1.2 Hz), 5.98 (s, 1H); ${ }^{13} \mathrm{C}$ NMR (50 MHz, $\left.\mathrm{CDCl}_{3}\right) \delta 69.1,116.5,124.3,125.1,129.1,129.7,132.0$, $134.2,134.3,147.9$.

2-[Hydroxy(3-nitrophenyl)methyl] acrylonitrile (3c)

IR (KBr) $v_{\max } / \mathrm{cm}^{-1} 3345,3105,2239,1583,1520,1348$; ${ }^{1} \mathrm{H}$ NMR $\left(200 \mathrm{MHz}, \mathrm{CDCl}_{3}\right) \delta 8.24(\mathrm{dd}, 1 \mathrm{H}, J 1.8 / 1.6 \mathrm{~Hz})$, 
8.18 (ddd, 1H, $J$ 8.0/1.0/1.2 Hz), 7.57 (t, $1 \mathrm{H}, J 8.0 \mathrm{~Hz}$ ), 7.75 (ddd, 1H, J 7.8/1.6 Hz), 6.09 (d, 1H, J 0.8 Hz), 6.20 (d, 1H, J 1.6 Hz), 5.43 (s, 1H), 3.02 (br s, 1H, CHOH); ${ }^{13} \mathrm{C} \mathrm{NMR}\left(50 \mathrm{MHz}, \mathrm{CDCl}_{3}\right) \delta 72.7,116.3,121.1,123.4$, 125.0, 129.7, 131.5, 132.5, 141.2, 148.0.

\section{2-[Hydroxy(4-nitrophenyl)methyl] acrylonitrile (3d)}

$\operatorname{IR}(\mathrm{KBr}) v_{\max } / \mathrm{cm}^{-1} 3447,3115,2228,1599,1520,1348$, 736; ' ${ }^{\mathrm{H}} \mathrm{NMR}\left(200 \mathrm{MHz}, \mathrm{CDCl}_{3}\right) \delta 8.21(\mathrm{~d}, 2 \mathrm{H}, J 8.8 \mathrm{~Hz})$, $7.58(\mathrm{~d}, 2 \mathrm{H}, J 9.0 \mathrm{~Hz}), 6.07(\mathrm{~d}, 1 \mathrm{H}, J 0.8 \mathrm{~Hz}), 6.16(\mathrm{~d}, 1 \mathrm{H}$, $J 0.6 \mathrm{~Hz}$ ), $5.42(\mathrm{~s}, 1 \mathrm{H}), 3.23(\mathrm{br} \mathrm{s}, 1 \mathrm{H}, \mathrm{CHOH}) ;{ }^{13} \mathrm{C} \mathrm{NMR}$ $\left(50 \mathrm{MHz}, \mathrm{CDCl}_{3}\right) \delta 73.0,116.6,123.9,126.1,127.3,130.5$, 146.8, 147.8.

\section{2-[Hydroxy(pyridin-2-yl)methyl] acrylonitrile (3e)}

IR (film) $v_{\max } / \mathrm{cm}^{-1} 3200,2225,1600 ;{ }^{1} \mathrm{H}$ NMR $\left(200 \mathrm{MHz}, \mathrm{CDCl}_{3}\right) \delta 8.56(\mathrm{ddd}, 1 \mathrm{H}, J 8.0 / 1.4 \mathrm{~Hz}$ ), 7.75 (ddd, $2 \mathrm{H}, J 7.8 / 7.6 / 1.6 \mathrm{~Hz}), 7.37(\mathrm{~d}, 1 \mathrm{H}, J 7.8 \mathrm{~Hz}), 7.29$ (ddd, 1H, $J$ 0.8/1.0/1.2 Hz), $5.28(\mathrm{~s}, 1 \mathrm{H}), 6.21(\mathrm{~s}, 1 \mathrm{H}), 6.05$ $(\mathrm{s}, 1 \mathrm{H}) ;{ }^{13} \mathrm{C} \mathrm{NMR}\left(50 \mathrm{MHz}, \mathrm{CDCl}_{3}\right) \delta 72.9,116.7,121.2$, 123.7, 125.8, 130.9, 137.4, 148.5, 156.0.

\section{2-[Hydroxy(pyridin-3-yl)methyl] acrylonitrile (3f)}

${ }^{1} \mathrm{H} \mathrm{NMR}\left(200 \mathrm{MHz}, \mathrm{CDCl}_{3}\right) \delta 8.41(\mathrm{~m}, 2 \mathrm{H}), 7.79$ (ddd, $1 \mathrm{H}, J 7.8 / 1.8 / 1.6 \mathrm{~Hz}), 7.33(\mathrm{dd}, 1 \mathrm{H}, J 8.0 \mathrm{~Hz}), 6.05(\mathrm{~d}$, $1 \mathrm{H}, J 1.0 \mathrm{~Hz}), 6.17(\mathrm{~d}, 1 \mathrm{H}, J 1.2), 5.33(\mathrm{~s}, 1 \mathrm{H}) ;{ }^{13} \mathrm{C} \mathrm{NMR}$ $\left(50 \mathrm{MHz}, \mathrm{CDCl}_{3}\right) \delta 71.5,116.7,124.1,126.0,130.4,135.0$, 136.1, 147.4, 148.9.

\section{2-[Hydroxy(pyridin-4-yl)methyl] acrylonitrile (3g)}

${ }^{1} \mathrm{H}$ NMR $\left(200 \mathrm{MHz}, \mathrm{CDCl}_{3}\right) \delta 8.50(\mathrm{~d}, 2 \mathrm{H}, J 6 \mathrm{~Hz})$, $7.36(\mathrm{~d}, 2 \mathrm{H}, J 5.8 \mathrm{~Hz}), 5.31(\mathrm{~s}, 1 \mathrm{H}), 6.16(\mathrm{~d}, 1 \mathrm{H}, J 0.6 \mathrm{~Hz})$, $6.06(\mathrm{~s}, 1 \mathrm{H}) ;{ }^{13} \mathrm{C} \mathrm{NMR}\left(50 \mathrm{MHz}, \mathrm{CDCl}_{3}\right) \delta 72.5,116.5$, 121.4, 125.7, 130.6, 149.1, 149.6.

2-[hydroxy (4-fluorphenyl) methyl] acrylonitrile (3h)

${ }^{1} \mathrm{H}$ NMR $\left(200 \mathrm{MHz}, \mathrm{CDCl}_{3}\right) \delta 7.39$ (dd, $2 \mathrm{H}, J$ 8.4, $5.2 \mathrm{~Hz}), 7.10(\mathrm{t}, 2 \mathrm{H}, J 8.4 \mathrm{~Hz}), 6.13(\mathrm{~d}, 1 \mathrm{H}, J 1.2 \mathrm{~Hz})$, $6.06(\mathrm{~d}, 1 \mathrm{H}, J 1.2 \mathrm{~Hz}), 5.32(\mathrm{~d}, 1 \mathrm{H}, J 4.0 \mathrm{~Hz}), 2.31(\mathrm{~d}$, $1 \mathrm{H}, J 4.0 \mathrm{~Hz}) ;{ }^{13} \mathrm{C}$ NMR $\left(50 \mathrm{MHz}, \mathrm{CDCl}_{3}\right) \delta 163.1,135.2$, 130.2, 128.5, 126.3, 117.0, 116.0, 73.6.

\section{2-[hydroxyl (4-chlorophenyl) methyl] acrylonitrile (3i)}

IR (KBr) $v_{\max } / \mathrm{cm}^{-1} 3441,2229$; ${ }^{1} \mathrm{H}$ NMR $(200 \mathrm{MHz}$, $\left.\mathrm{CDCl}_{3}\right) \delta 7.39(2 \mathrm{H}, J 8.8 \mathrm{~Hz}), 7.35(2 \mathrm{H}, J 8.8 \mathrm{~Hz}), 6.13(\mathrm{~d}$, $1 \mathrm{H}, J 1.6 \mathrm{~Hz}), 6.06(\mathrm{~d}, 1 \mathrm{H}, J 1.6 \mathrm{~Hz}), 5.32(\mathrm{~m}, 1 \mathrm{H}), 2.30(\mathrm{~d}$, $1 \mathrm{H}, J 4.0 \mathrm{~Hz}) ;{ }^{13} \mathrm{C}$ NMR $\left(50 \mathrm{MHz}, \mathrm{CDCl}_{3}\right) \delta 137.8,135.1$, 130.3, 129.3, 128.1, 126.1, 116.9, 73.7.

2-[Hydroxy(4-bromophenyl)methyl] acrylonitrile (3j)

${ }^{1} \mathrm{H}$ NMR $\left(200 \mathrm{MHz}, \mathrm{CDCl}_{3}\right) \delta 7.54(\mathrm{dd}, 2 \mathrm{H}$,
$J$ 6.0/2.0 Hz), $7.28(\mathrm{dd}, 2 \mathrm{H}, J 6.0 / 1.8 \mathrm{~Hz}), 6.11(\mathrm{~d}, 1 \mathrm{H}$, $J 1.6 \mathrm{~Hz}), 6.04(\mathrm{~d}, 1 \mathrm{H}, J 1.0 \mathrm{~Hz}), 5.27(\mathrm{~s}, 1 \mathrm{H}), 2.71(\mathrm{~s}$, $\mathrm{CHOH}) ;{ }^{13} \mathrm{C} \mathrm{NMR}\left(50 \mathrm{MHz}, \mathrm{CDCl}_{3}\right) \delta 73.3,116.7,122.7$, 125.8, 128.1, 130.3, 131.9, 138.1 .

\section{2-[Hydroxy(naphth-2-yl)methyl] acrylonitrile (3k)}

${ }^{1} \mathrm{H}$ NMR $\left(200 \mathrm{MHz}, \mathrm{CDCl}_{3}\right) \delta$ 7.42-7.89 (m, 7H), $6.15(\mathrm{~d}, 2 \mathrm{H}, J 1.4 \mathrm{~Hz}), 6.06(\mathrm{~d}, 2 \mathrm{H}, J 1.6 \mathrm{~Hz}), 5.46(\mathrm{~d}$, $1 \mathrm{H}, J 3.8 \mathrm{~Hz}), 2.50(\mathrm{~d}, 1 \mathrm{H}, J 3.8 \mathrm{~Hz}) ;{ }^{13} \mathrm{C} \mathrm{NMR}(50 \mathrm{MHz}$, $\left.\mathrm{CDCl}_{3}\right) \delta 137.8,134.9,134.5,131.6,130.4,129.6,129.18$, 129.16, 128.0, 127.5, $127.4,125.2,118.4,75.9$.

2-[hydroxyl (1,3-dioxolephenyl) methyl] acrylonitrile (3l)

IR (KBr) $v_{\text {max }} / \mathrm{cm}^{-1} 3471,2225 ;{ }^{1} \mathrm{H}$ NMR $(200 \mathrm{MHz}$, $\left.\mathrm{CDCl}_{3}\right) \delta$ 6.9-6.6 (m, 3H) $6.1(\mathrm{~d}, 1 \mathrm{H}, J 1.46 \mathrm{~Hz}), 6.0(\mathrm{~d}$, $1 \mathrm{H}, J 1.46 \mathrm{~Hz}), 5.82(\mathrm{~s}, 2 \mathrm{H}), 5.20(\mathrm{~s}, 1 \mathrm{H}), 2.6(\mathrm{~s}, 1 \mathrm{H})$.

\section{Methyl 2-[(hydroxylphenyl)methyl] acrylate (6a)}

IR (KBr) $v_{\text {max }} / \mathrm{cm}^{-1} 3344,1716$; ${ }^{1} \mathrm{H}$ NMR $(200 \mathrm{MHz}$, $\left.\mathrm{CDCl}_{3}\right) \delta 7.40-7.29(\mathrm{~m}, 5 \mathrm{H}), 6.34(\mathrm{~s}, 1 \mathrm{H}), 5.83(\mathrm{~s}, 1 \mathrm{H})$, $5.58(\mathrm{~d}, 1 \mathrm{H}, J 5.6 \mathrm{~Hz}), 3.73(\mathrm{~s}, 3 \mathrm{H}), 2.98(\mathrm{~d}, 1 \mathrm{H}, J 5.6 \mathrm{~Hz})$; ${ }^{13} \mathrm{C} \mathrm{NMR}\left(50 \mathrm{MHz}, \mathrm{CDCl}_{3}\right) \delta 166.9,142.2,141.5,128.6$, 128.0, 126.8, 126.2, 73.3, 52.1.

Methyl 2-[hydroxyl (2-nitrophenyl)methyl] acrylate (6b)

${ }^{1} \mathrm{H}$ NMR $\left(200 \mathrm{MHz}, \mathrm{CDCl}_{3}\right) \delta 8.82(\mathrm{~d}, 1 \mathrm{H}, J 8.47 \mathrm{~Hz})$, 8.56 (t, $1 \mathrm{H}, J 7.55 / 7.35 \mathrm{~Hz}), 8.42$ (t, $1 \mathrm{H}, J 8.47 / 7.35 \mathrm{~Hz})$, $8.33(\mathrm{~d}, 1 \mathrm{H} J 8.47 \mathrm{~Hz}), 6.36(\mathrm{~s}, 1 \mathrm{H}), 6.31(\mathrm{~s}, 1 \mathrm{H}), 5.59(\mathrm{~d}$, $1 \mathrm{H}, J 5.3 \mathrm{~Hz}, \mathrm{CHOH}), 3.74$ (s, $3 \mathrm{H}) ;{ }^{13} \mathrm{C}$ NMR $(50 \mathrm{MHz}$, $\left.\mathrm{CDCl}_{3}\right) \delta 52.2,67.6,124.6,126.5,128.7,133.5,136.0$, $140.7,148.3,166.4$.

\section{Methyl 2-[hydroxyl(3-nitrophenyl)methyl] acrylate (6c)}

${ }^{1} \mathrm{H}$ NMR $\left(200 \mathrm{MHz}, \mathrm{CDCl}_{3}\right) \delta 8.48(\mathrm{~d}, 1 \mathrm{H}, J 2.0 \mathrm{~Hz})$, 8.39 (dd, $1 \mathrm{H}, J 5.0 / 1.5 \mathrm{~Hz}), 7.74$ (ddd, $1 \mathrm{H}, J 8.0 / 2.0 / 2.0 \mathrm{~Hz}$ ), $7.28(\mathrm{~m}, 1 \mathrm{H}), 6.40(\mathrm{~d}, 1 \mathrm{H}, J 0.6), 6.01(\mathrm{sl}, 1 \mathrm{H}) ; 5.60(\mathrm{sl}$, $1 \mathrm{H}), 3,71(\mathrm{~s}, 3 \mathrm{H}) ;{ }^{13} \mathrm{C} \mathrm{NMR}\left(50 \mathrm{MHz}, \mathrm{CDCl}_{3}\right) \delta 52.0,70.5$, 123.4, 126.1, 134.7, 137.6, 141.6, 148.2, 148.4, 166.2.

\section{Methyl 2-[hydroxyl(4-nitrophenyl)methyl]acrylate (6d)}

${ }^{1} \mathrm{H} \mathrm{NMR}\left(200 \mathrm{MHz}, \mathrm{CDCl}_{3}\right) \delta 1.57(\mathrm{~s}, 1 \mathrm{H}), 3.74(\mathrm{~s}, 3 \mathrm{H})$, $5.63(\mathrm{~d}, 1 \mathrm{H}, J 6.32 \mathrm{~Hz}), 5.87(\mathrm{~s}, 1 \mathrm{H}), 6.40(\mathrm{~s}, 1 \mathrm{H}), 7.61(\mathrm{~m}$, $2 \mathrm{H}), 8.25(\mathrm{~m}, 2 \mathrm{H}) ;{ }^{13} \mathrm{C} \mathrm{NMR}\left(50 \mathrm{MHz}, \mathrm{CDCl}_{3}\right) \delta 52.1,72.5$, 123.5, 127.1, 127.2, 140.9, 147.3, 148.5, 166.2.

\section{Methyl 2-[hydroxyl(2-pyridinyl)methyl] acrylate (6e)}

${ }^{1} \mathrm{H}$ NMR $\left(200 \mathrm{MHz}, \mathrm{CDCl}_{3}\right) \delta 8.51(\mathrm{~d}, 1 \mathrm{H}, J 4.4 \mathrm{~Hz})$, 7.66 (ddd, 1H, J 7.8/1.6 Hz), 7.4 (d, 1H, $J 8.0 \mathrm{~Hz}), 7.21$ (m, 1H), $6.34(\mathrm{sl}, 1 \mathrm{H}), 5.96(\mathrm{sl}, 1 \mathrm{H}), 5.61(\mathrm{sl}, 1 \mathrm{H}), 3.71$ $(\mathrm{s}, 3 \mathrm{H}) ;{ }^{13} \mathrm{C} \mathrm{NMR}\left(50 \mathrm{MHz}, \mathrm{CDCl}_{3}\right) \delta 51.8,72.0,121.2$, 122.6, 126.8, 136.8, 141.5, 148.1, 159.4, 166.4 . 
Methyl 2-[hydroxyl(3-pyridinyl)methyl] acrylate ( $6 \mathrm{f})$

${ }^{1} \mathrm{H}$ NMR $\left(200 \mathrm{MHz}, \mathrm{CDCl}_{3}\right) \delta 8.48(\mathrm{~d}, 1 \mathrm{H}, J 2.0 \mathrm{~Hz})$, 8.39 (dd, $1 \mathrm{H}, J 5.0 / 1.5 \mathrm{~Hz}), 7.74$ (ddd, $1 \mathrm{H}, J 8.0 / 2.0 / 2.0 \mathrm{~Hz}$ ), $7.28(\mathrm{~m}, 1 \mathrm{H}), 6.40(\mathrm{~d}, 1 \mathrm{H}, J 0.6), 6.01(\mathrm{sl}, 1 \mathrm{H}), 5.60(\mathrm{sl}$, $1 \mathrm{H}), 3.71(\mathrm{~s}, 3 \mathrm{H}) ;{ }^{13} \mathrm{C} \mathrm{NMR}\left(50 \mathrm{MHz}, \mathrm{CDCl}_{3}\right) \delta 52.0,70.5$, 123.4, 126.1, 134.7, 137.6, 141.6, 148.2, 148.4, 166.2.

Methyl 2-[hydroxyl (4-pyridinyl)methyl] acrylate (6g)

${ }^{1} \mathrm{HNMR}\left(200 \mathrm{MHz}, \mathrm{CDCl}_{3}\right) \delta 8.47(\mathrm{dd}, 2 \mathrm{H}, J 4.6 / 1.6 \mathrm{~Hz})$, 7.30 (dd, $2 \mathrm{H}, J 4.6 / 1.6 \mathrm{~Hz}), 5.89$ (s, 1H), 5.51 (s, 1H), 3.70 $(\mathrm{s}, 1 \mathrm{H}) ;{ }^{13} \mathrm{C}$ NMR $\left(50 \mathrm{MHz}, \mathrm{CDCl}_{3}\right) \delta 52.1,71.9,121.5$, 127.1, 140.9, 149.3, 151.0, 166.3.

Methyl 2-[hydroxyl (4-fluorphenyl)methyl] acrylate (6h)

${ }^{1} \mathrm{HNMR}\left(200 \mathrm{MHz}, \mathrm{CDCl}_{3}\right) \delta 7.35(\mathrm{dd}, 2 \mathrm{H}, J 8.8,6.0 \mathrm{~Hz})$, $7.03(\mathrm{t}, 2 \mathrm{H}, J 8.8 \mathrm{~Hz}), 6.34(\mathrm{~s}, 1 \mathrm{H}), 5.82(\mathrm{~s}, 1 \mathrm{H}), 5.55(\mathrm{~d}$, $1 \mathrm{H}, J 5.6 \mathrm{~Hz}), 3.74(\mathrm{~s}, 3 \mathrm{H}), 2.99(\mathrm{~d}, 1 \mathrm{H}, J 5.6 \mathrm{~Hz}) ;{ }^{13} \mathrm{C}$ $\operatorname{NMR}\left(50 \mathrm{MHz}, \mathrm{CDCl}_{3}\right) \delta 166.9,162.6,142.1,137.2,128.5$, $126.3,115.5,72.9,52.2$.

Methyl 2-[hydroxyl (4-chlorophenyl)methyl] acrylate (6i)

IR (KBr) $v_{\text {max }} / \mathrm{cm}^{-1} 3255,1720 ;{ }^{1} \mathrm{H}$ NMR $(200 \mathrm{MHz}$, $\left.\mathrm{CDCl}_{3}\right) \delta 7.32(\mathrm{~s}, 4 \mathrm{H}), 6.35(\mathrm{~s}, 1 \mathrm{H}), 5.82(\mathrm{~s}, 1 \mathrm{H}), 5.54$ $(\mathrm{d}, 1 \mathrm{H}, J 6.0 \mathrm{~Hz}), 3.74(\mathrm{~s}, 3 \mathrm{H}), 3.03(\mathrm{~d}, 1 \mathrm{H}, J 6.0 \mathrm{~Hz})$; ${ }^{13} \mathrm{C}$ NMR $\left(50 \mathrm{MHz}, \mathrm{CDCl}_{3}\right) \delta 166.8,141.8,140.0,133.8$, $128.8,128.2,126.6,72.9,52.3$.
Methyl 2-[hydroxyl(4-bromophenyl)methyl] acrylate (6j)

${ }^{1} \mathrm{H}$ NMR $\left(200 \mathrm{MHz}, \mathrm{CDCl}_{3}\right) \delta 7.38(\mathrm{~d}, 2 \mathrm{H}, J 8.4 \mathrm{~Hz})$, $7.16(\mathrm{~d}, 2 \mathrm{H}, J 8.4 \mathrm{~Hz}), 6.26(\mathrm{~s}, 1 \mathrm{H}), 5.78(\mathrm{~s}, 1 \mathrm{H}), 5.41$ (s, 1H), 3.63 (s, 3H), 3.39 (bs, 1H); ${ }^{13} \mathrm{C}$ NMR $(50 \mathrm{MHz}$, $\left.\mathrm{CDCl}_{3}\right) \delta 166.4,141.5,140.3,131.3,128.3,126.1,121.6$, 72.3, 51.9 .

Methyl 2-[hydroxyl(2-naphthyl)methyl] acrylate (6k)

${ }^{1} \mathrm{H} \mathrm{NMR}\left(200 \mathrm{MHz}, \mathrm{CDCl}_{3}\right) \delta 1.57(\mathrm{~s}, 1 \mathrm{H}), 3.73(\mathrm{~s}, 3 \mathrm{H})$, $5.75(\mathrm{~d}, 1 \mathrm{H}, J 5.77 \mathrm{~Hz}), 5.88(\mathrm{t}, 1 \mathrm{H}, J 1.14 / 1.10 \mathrm{~Hz}), 6.39$ (s, 1H), $7.50(\mathrm{~m}, 3 \mathrm{H}), 7.78(\mathrm{~m}, 4 \mathrm{H}) ;{ }^{13} \mathrm{C}$ NMR $(50 \mathrm{MHz}$, $\left.\mathrm{CDCl}_{3}\right) \delta 51.9,73.2,124.5,125.4,125.9,126.0,126.8$, 127.5, 128.0, 128.1, 132.9, 133.1, 138.5, 141.8, 166.7.

Methyl 2-[hydroxyl(1,3-dioxolephenyl)methyl] acrylate (6l)

${ }^{1} \mathrm{H}$ NMR $\left(200 \mathrm{MHz}, \mathrm{CDCl}_{3}\right) \delta 1.57(\mathrm{~s}, 1 \mathrm{H}), 3.73(\mathrm{~s}$, $3 \mathrm{H}), 5.49(\mathrm{~d}, 1 \mathrm{H}, J 5 \mathrm{~Hz}), 5.86(\mathrm{t}, 1 \mathrm{H}, J 1.26 / 1.21 \mathrm{~Hz}), 5.96$ $(\mathrm{d}, 2 \mathrm{H}, J 0.86 \mathrm{~Hz}), 6.33$ (t, 1H, $J 1.09 / 0.8 \mathrm{~Hz}), 6.87(\mathrm{~m}$, $3 \mathrm{H}) ;{ }^{13} \mathrm{C}$ NMR $\left(50 \mathrm{MHz}, \mathrm{CDCl}_{3}\right) \delta 51.8,72.7,100.9,107.0$, 107.9, 120.0, 125.6, 135.2, 141.8, 147.0, 147.5, 166.5.

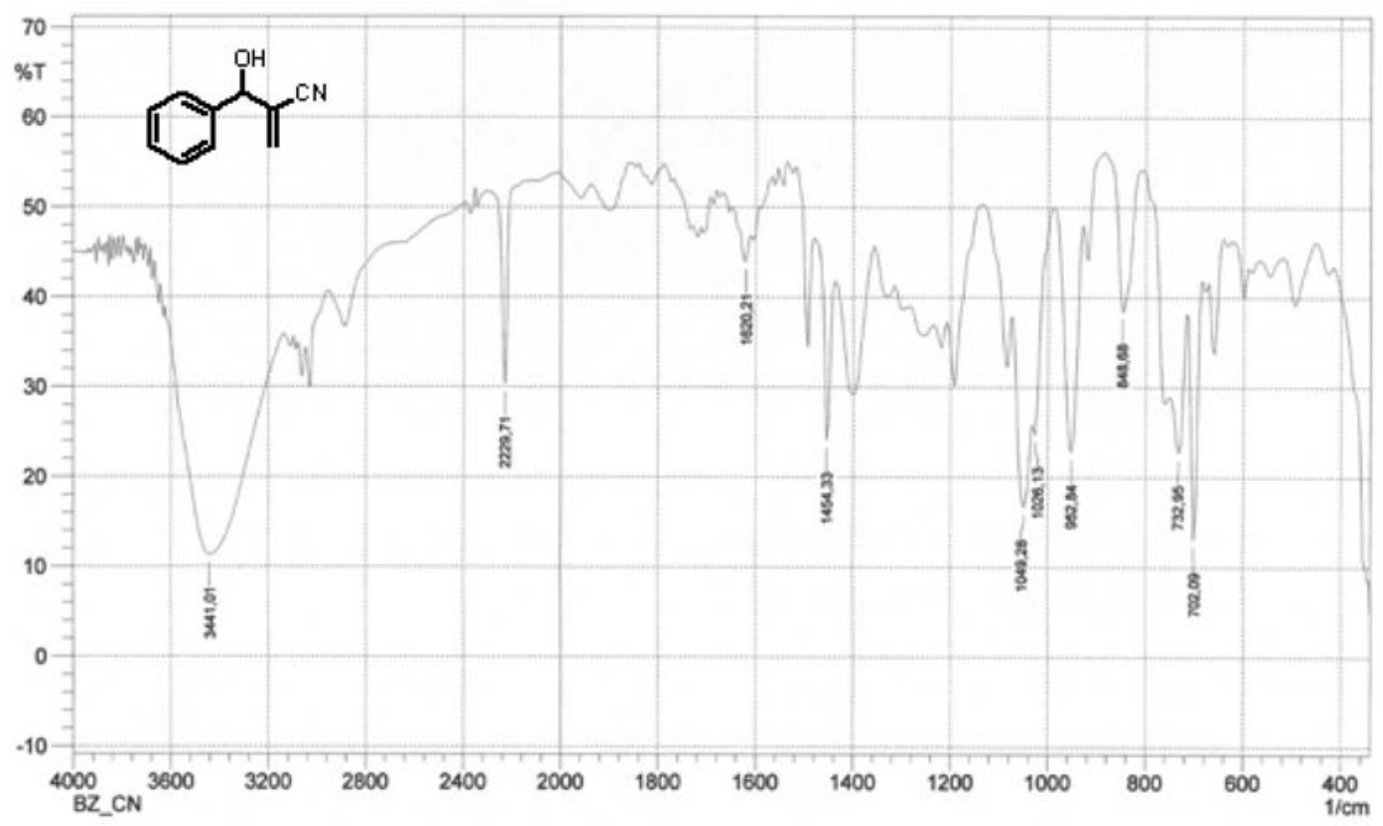

Figure S1. IR (KBr) of 2-[(Hydroxyphenyl)methyl] acrylonitrile (3a). 


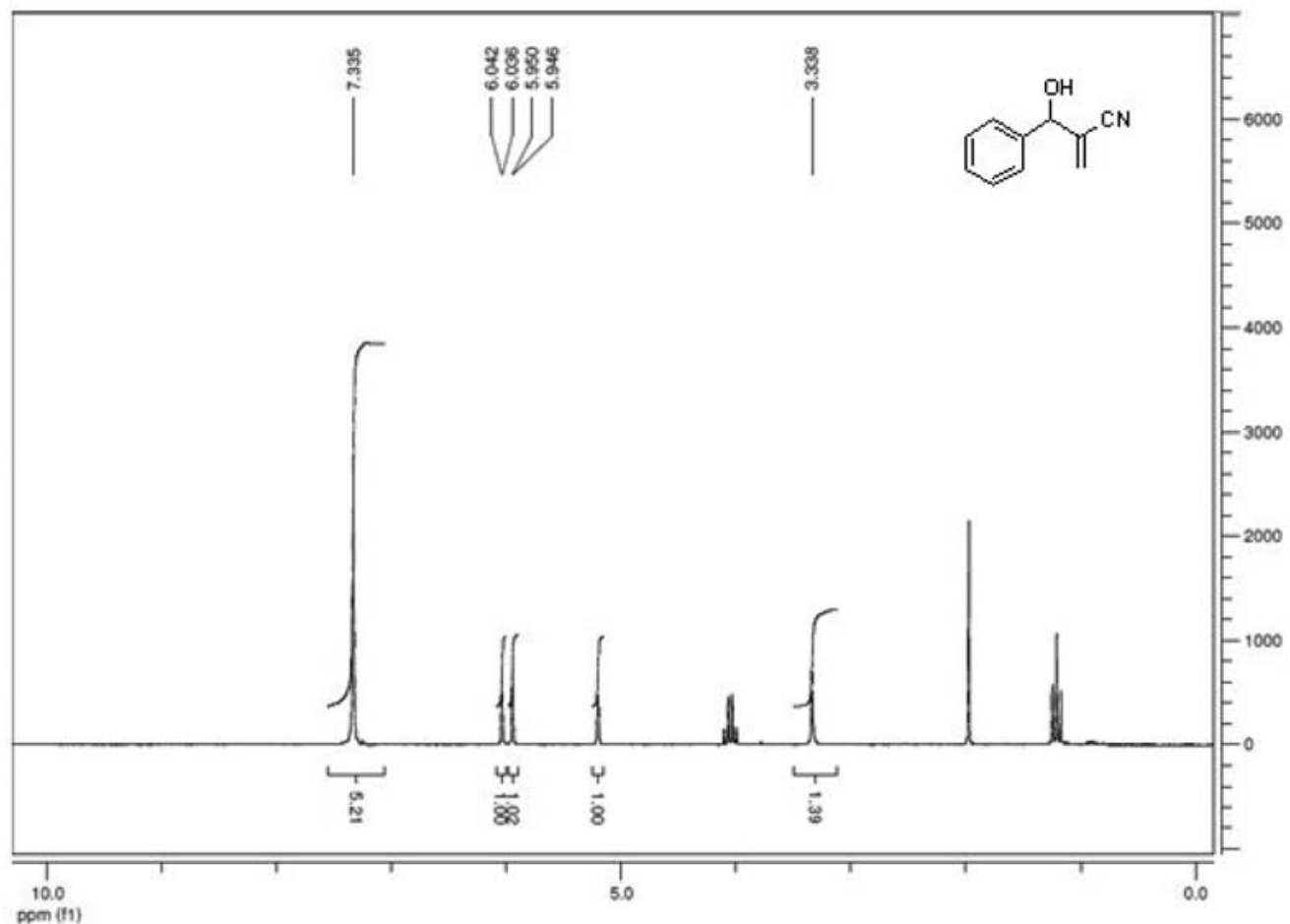

Figure S2. ${ }^{1} \mathrm{H} \mathrm{NMR}\left(\mathrm{CDCl}_{3}\right)$ spectrum of 2-[(Hydroxyphenyl)methyl] acrylonitrile (3a).

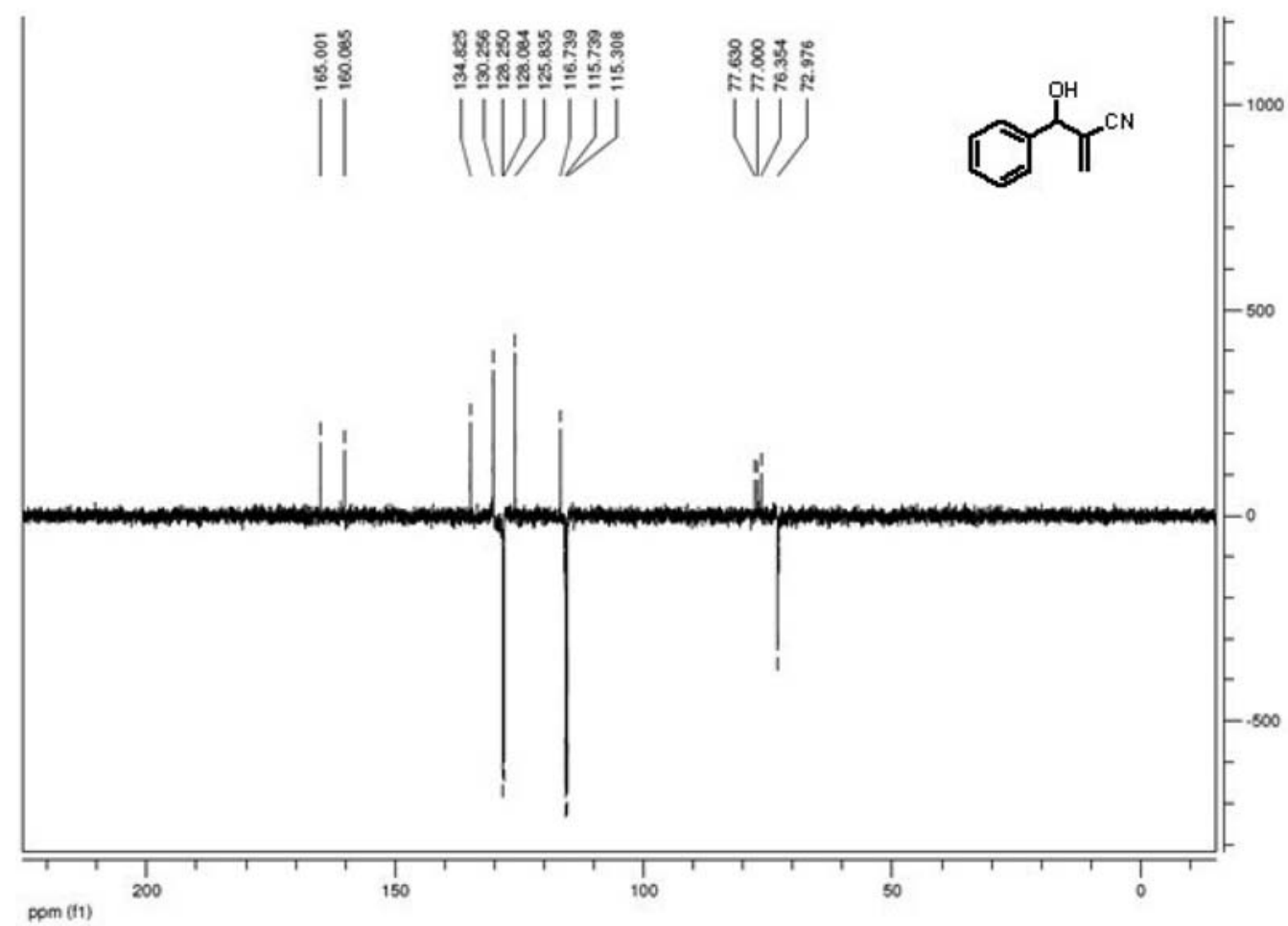

Figure S3. ${ }^{13} \mathrm{C}$ NMR $\left(\mathrm{CDCl}_{3}\right)$ spectrum of 2-[(Hydroxyphenyl)methyl] acrylonitrile (3a). 


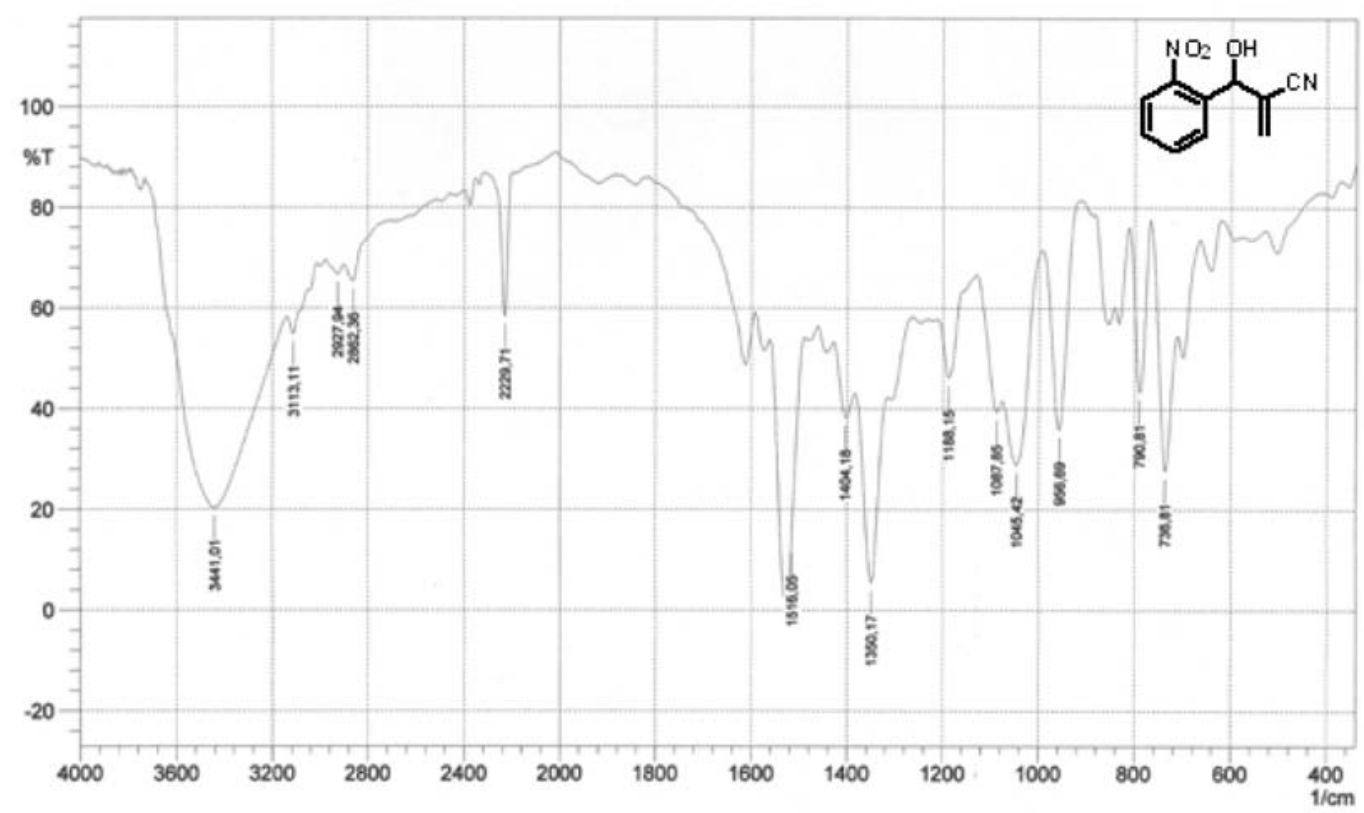

Figure S4. IR (KBr) of 2-[Hydroxy(2-nitrophenyl)methyl] acrylonitrile (3b).

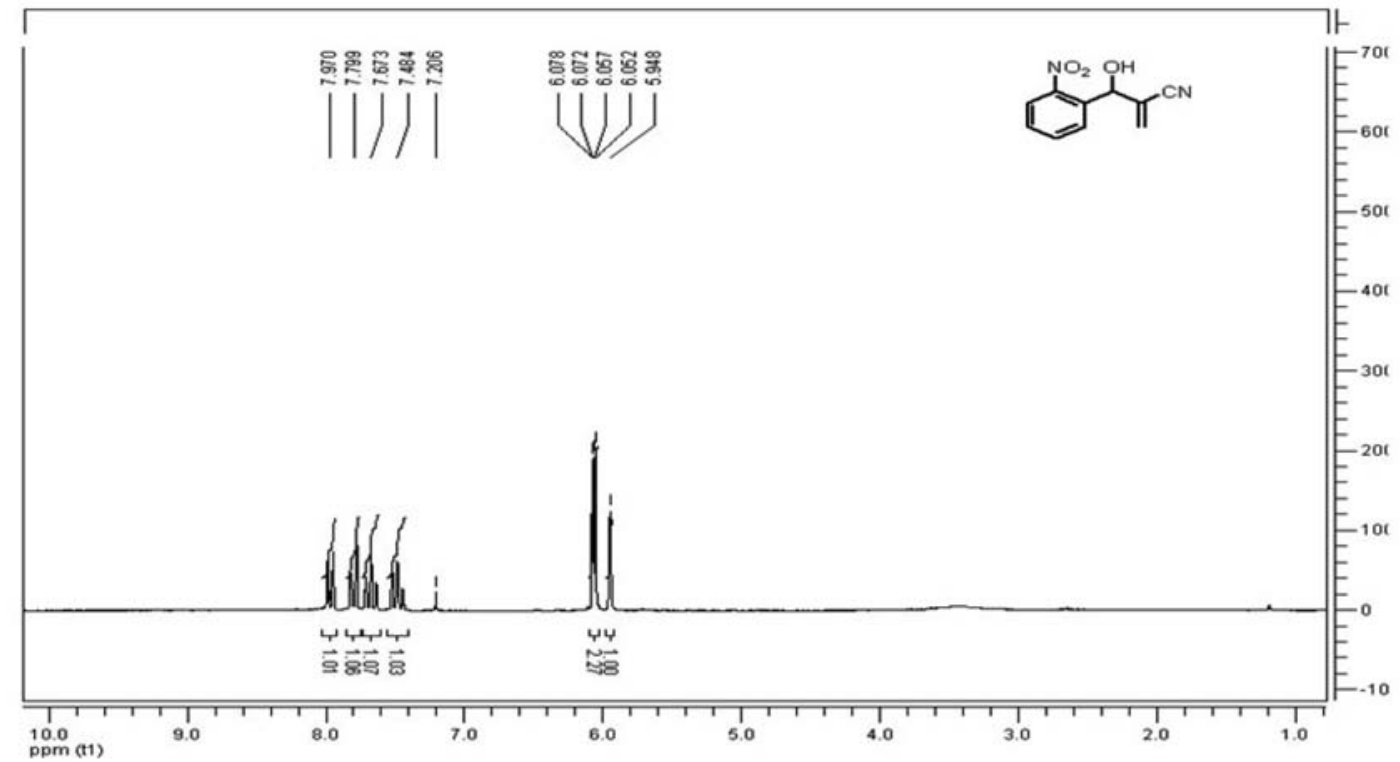

Figure S5. ${ }^{1} \mathrm{H}$ NMR $\left(\mathrm{CDCl}_{3}\right)$ spectrum of 2-[Hydroxy(2-nitrophenyl)methyl] acrylonitrile (3b) 


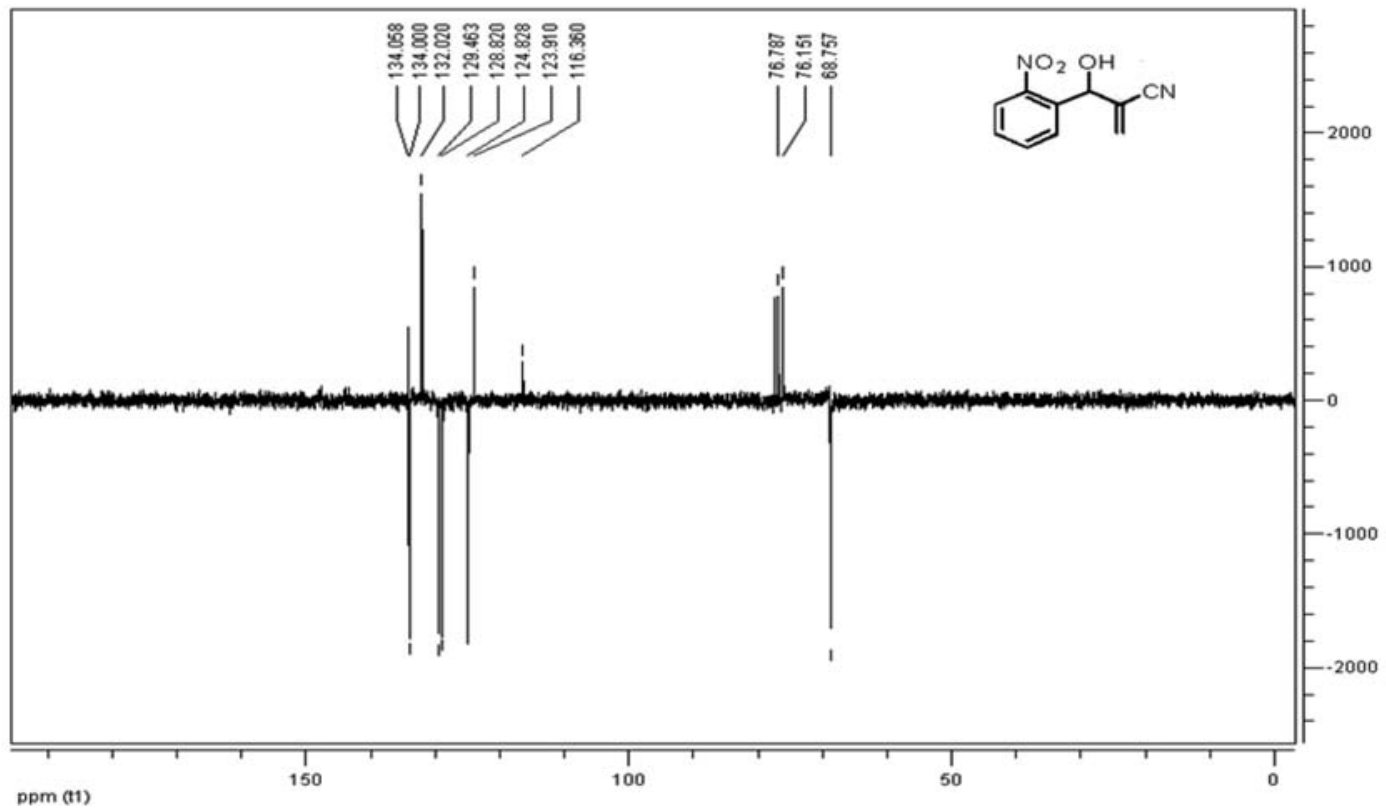

Figure S6. ${ }^{13} \mathrm{C}$ NMR $\left(\mathrm{CDCl}_{3}\right)$ spectrum of 2-[Hydroxy(2-nitrophenyl)methyl] acrylonitrile (3b).

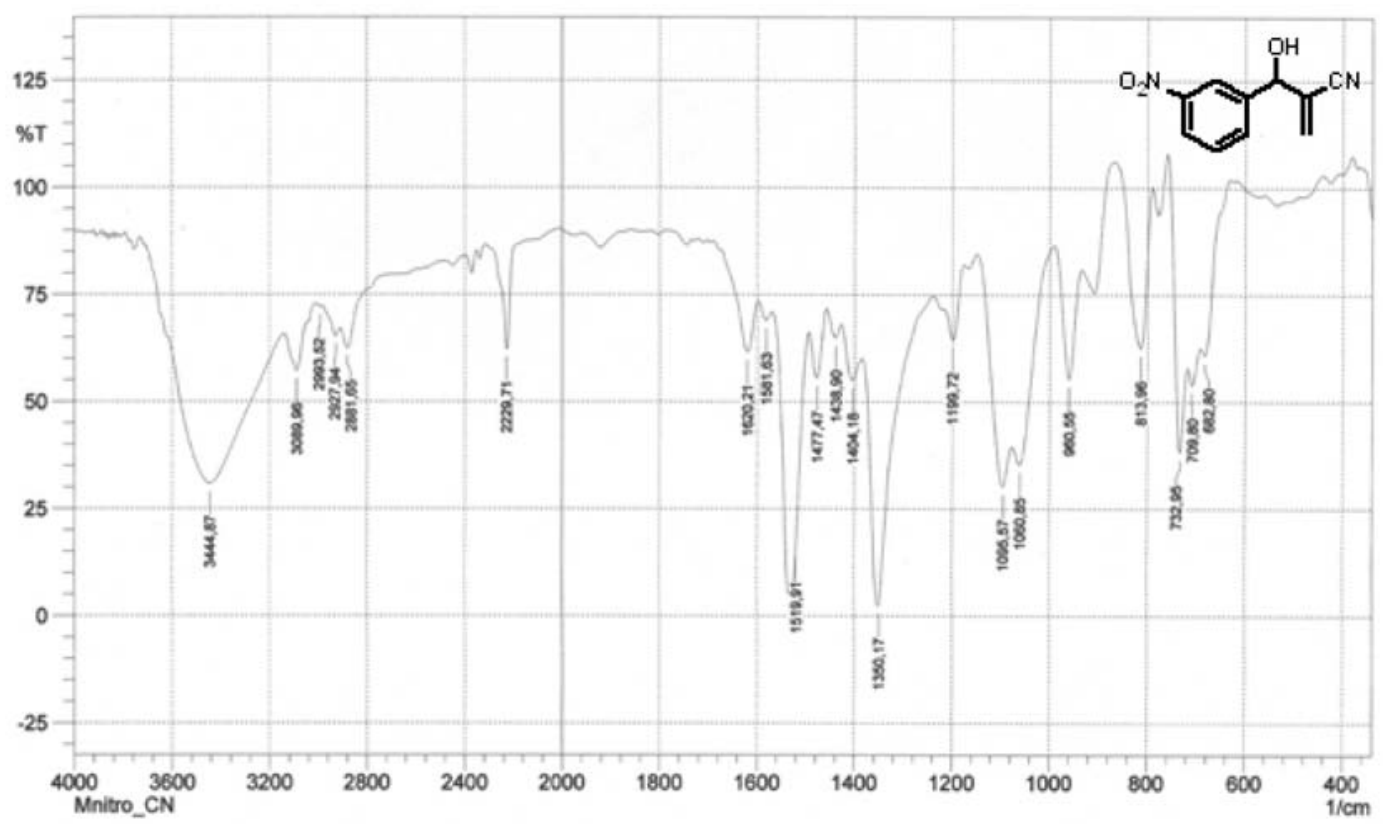

Figure S7. IR (KBr) of 2-[Hydroxy(3-nitrophenyl)methyl] acrylonitrile (3c). 


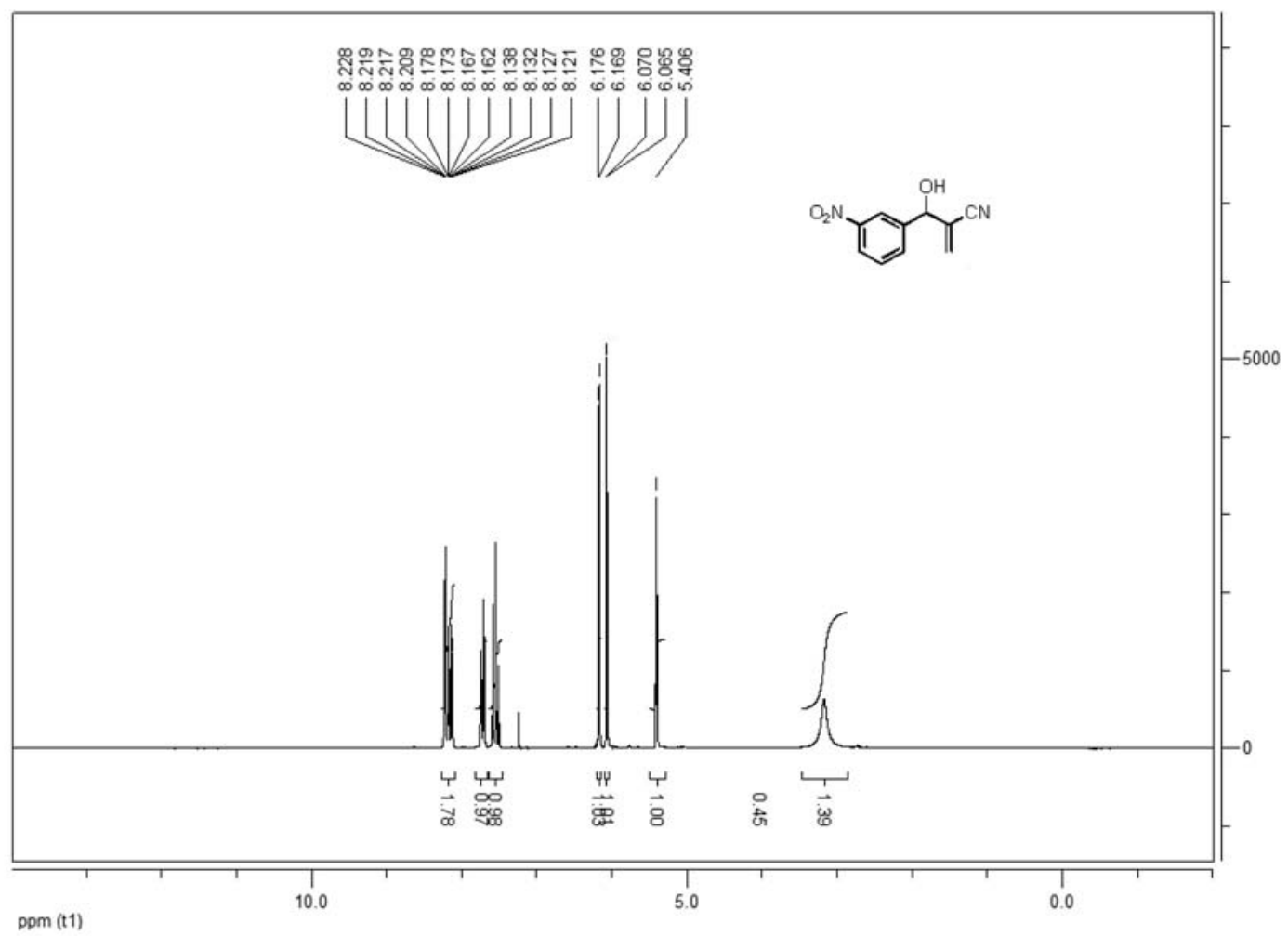

Figure S8. ${ }^{1} \mathrm{H} \mathrm{NMR}\left(\mathrm{CDCl}_{3}\right)$ spectrum of 2-[Hydroxy(3-nitrophenyl)methyl] acrylonitrile (3c).

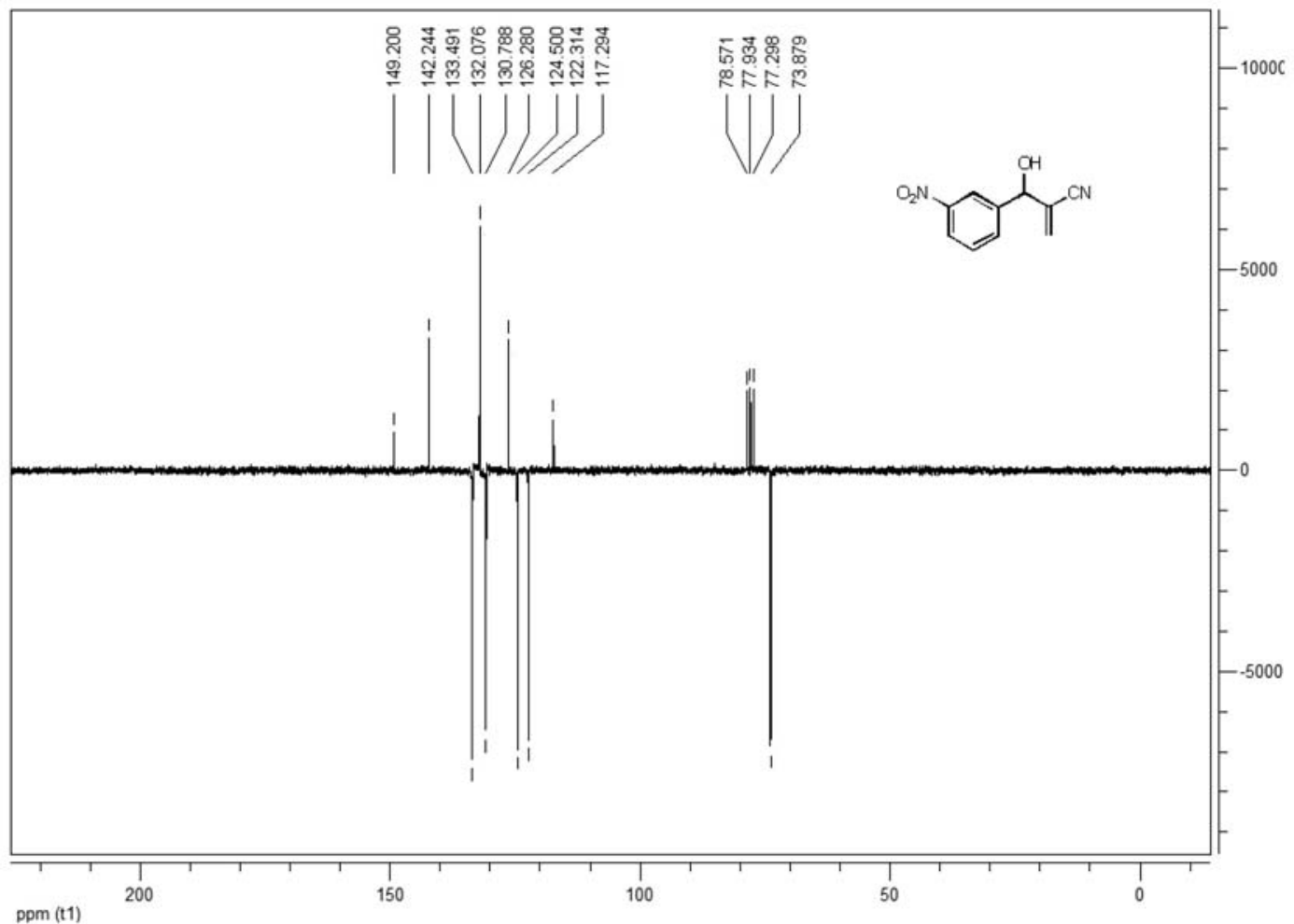

Figure S9. ${ }^{13 \mathrm{C}} \mathrm{NMR}\left(\mathrm{CDCl}_{3}\right)$ spectrum of 2-[Hydroxy(3-nitrophenyl)methyl] acrylonitrile (3c). 


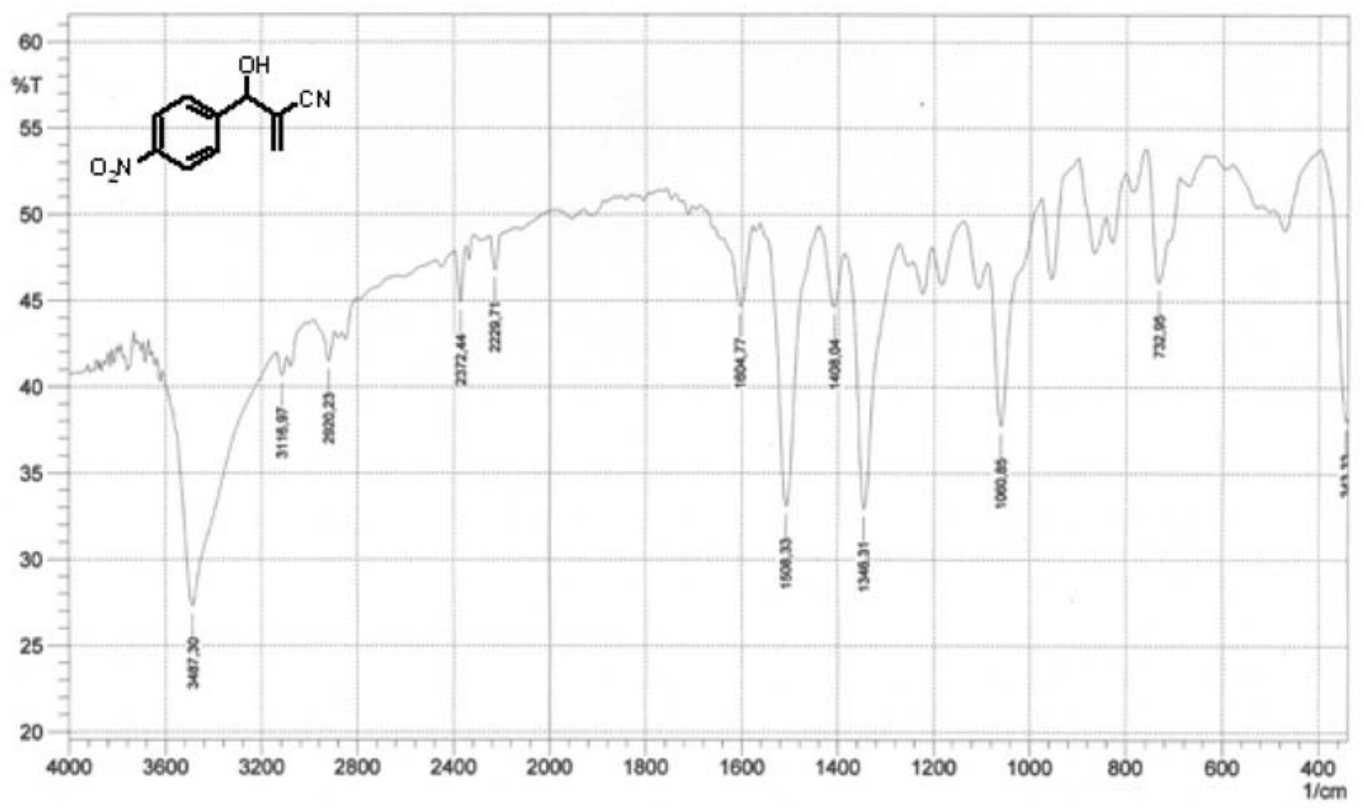

Figure S10. IR (KBr) of 2-[Hydroxy(4-nitrophenyl)methyl] acrylonitrile (3d).

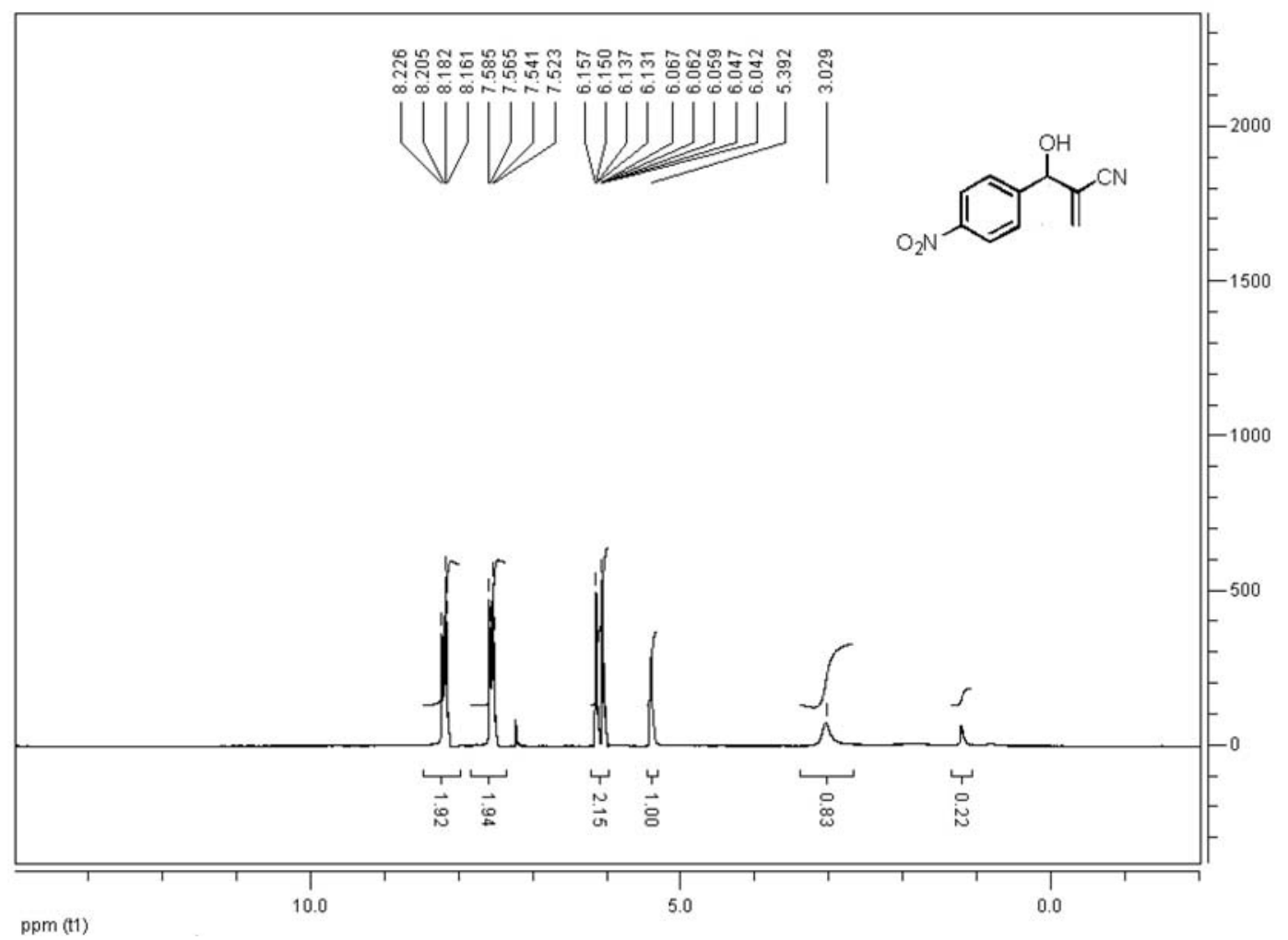

Figure S11. ${ }^{1} \mathrm{H}$ NMR $\left(\mathrm{CDCl}_{3}\right)$ spectrum of 2-[Hydroxy(4-nitrophenyl)methyl] acrylonitrile (3d). 


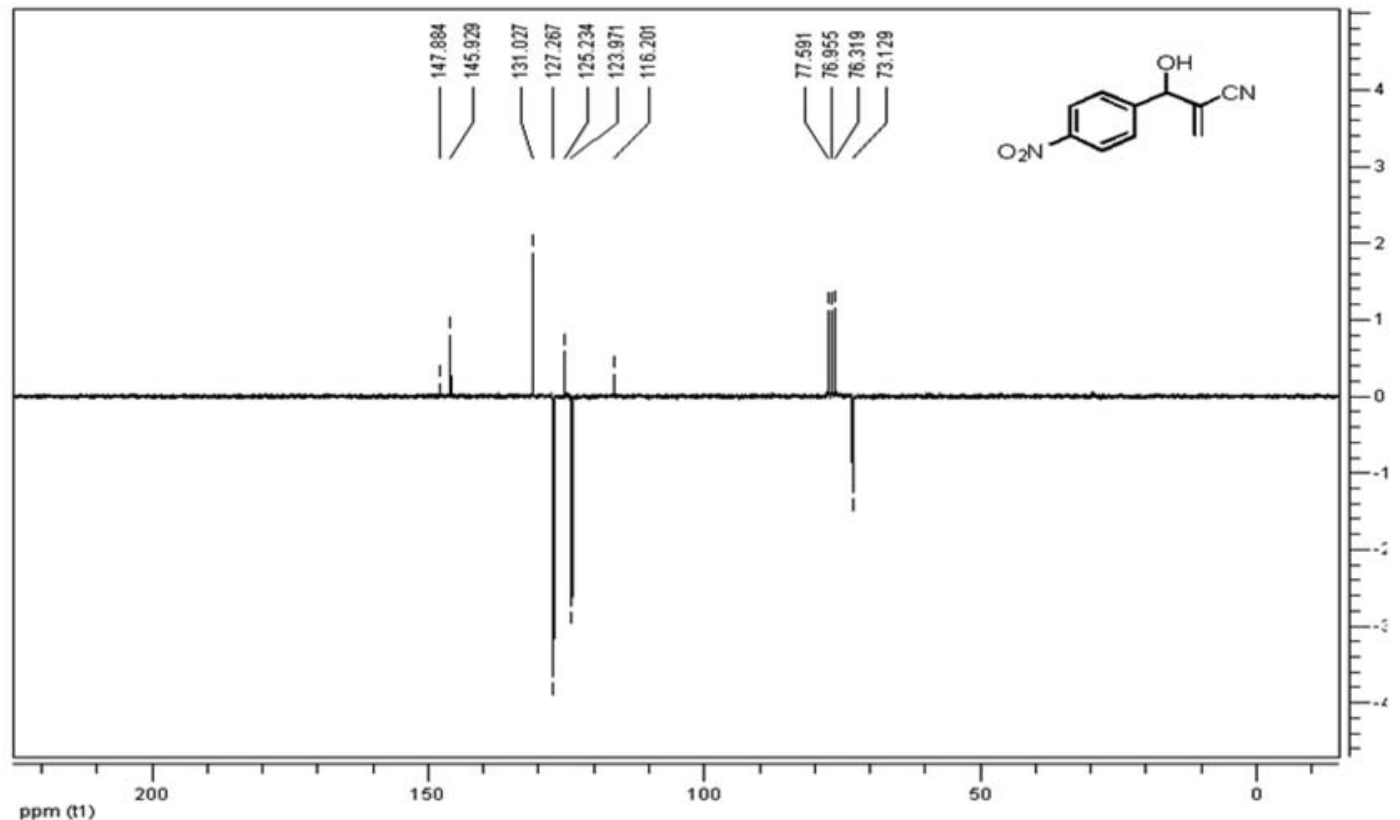

Figure S12. ${ }^{13} \mathrm{C}$ NMR $\left(\mathrm{CDCl}_{3}\right)$ spectrum of 2-[Hydroxy(4-nitrophenyl)methyl] acrylonitrile (3d).

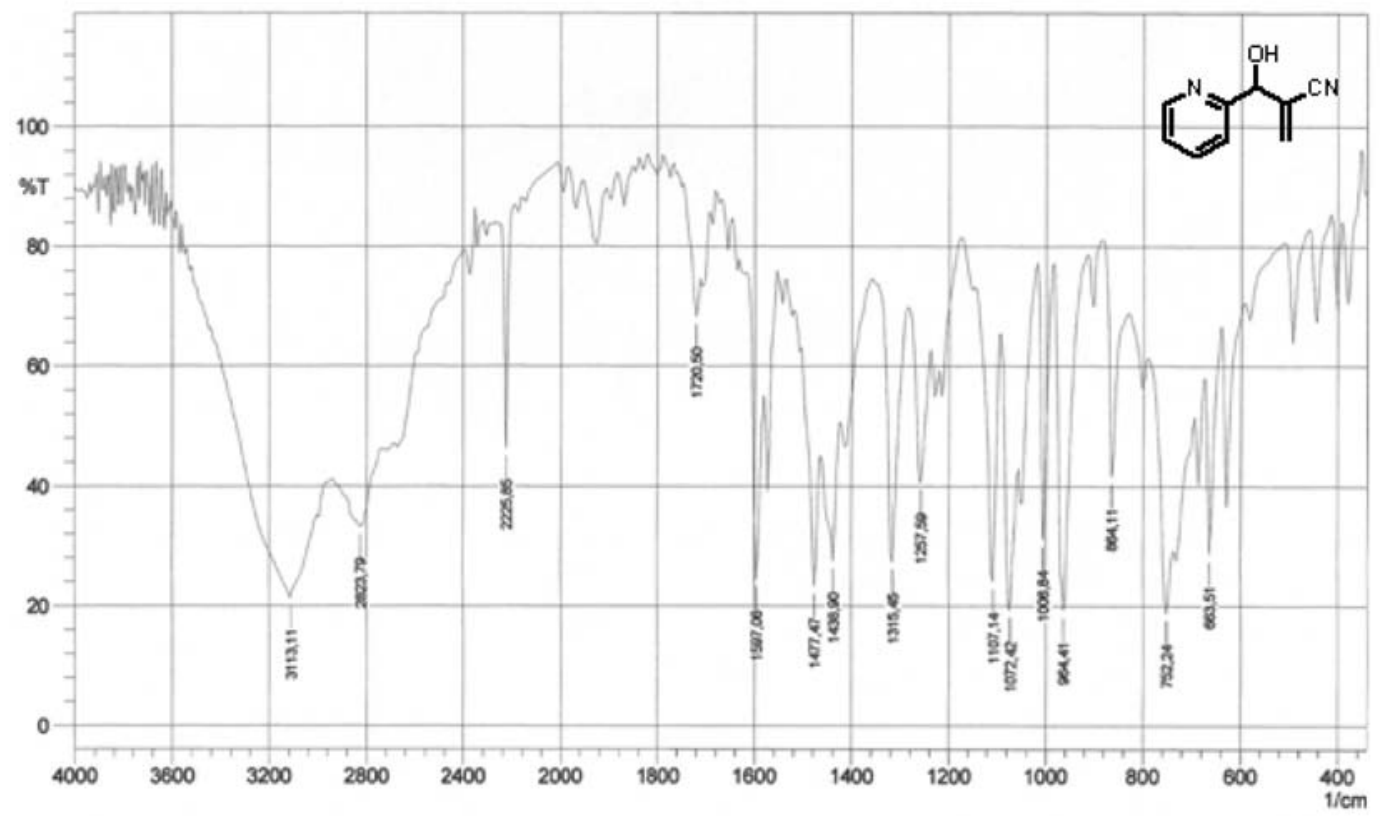

Figure S13. IR (KBr) of 2-[Hydroxy(pyridin-2-yl)methyl] acrylonitrile (3e). 


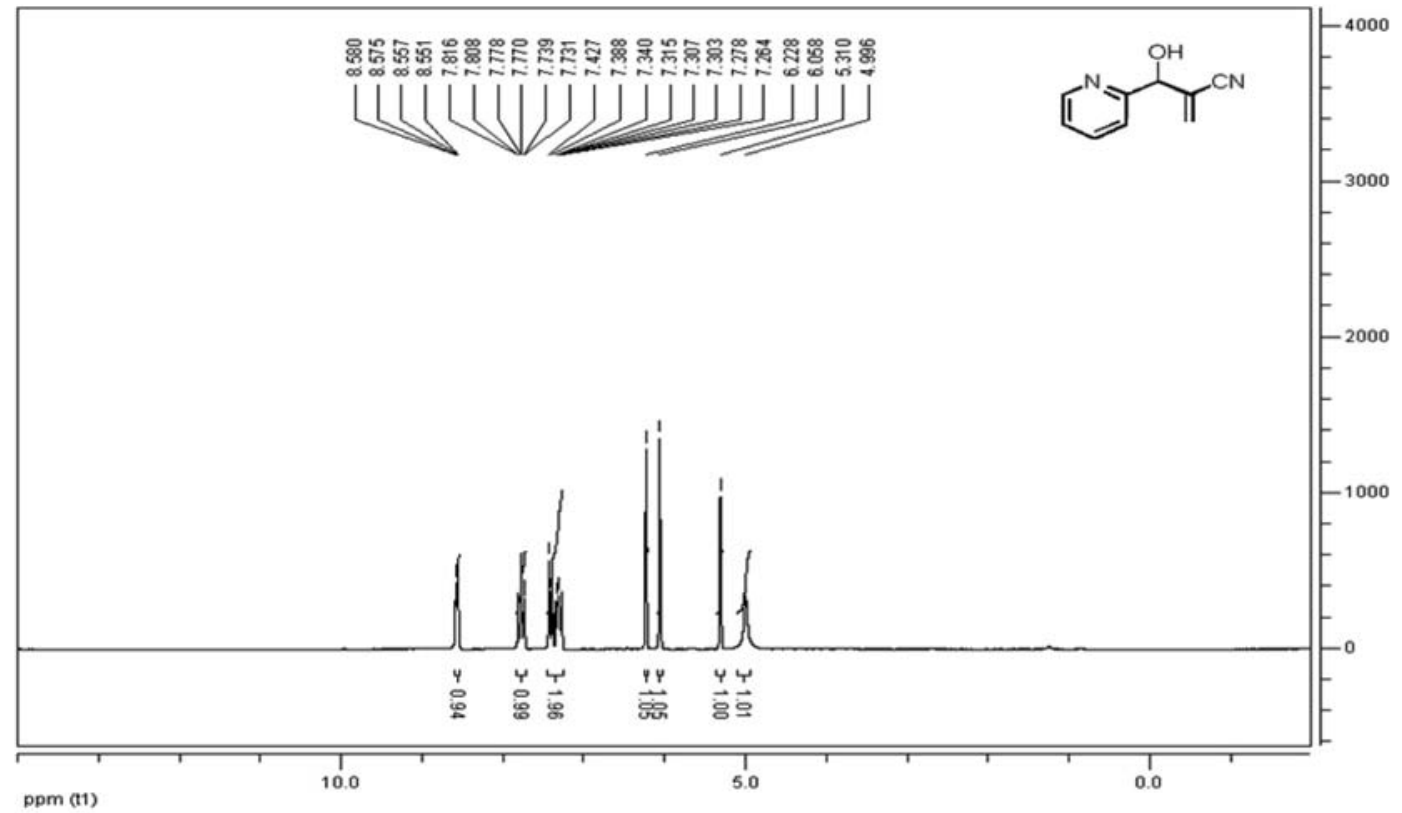

Figure S14. ${ }^{1} \mathrm{H}$ NMR $\left(\mathrm{CDCl}_{3}\right)$ spectrum of 2-[Hydroxy(pyridin-2-yl)methyl] acrylonitrile (3e).

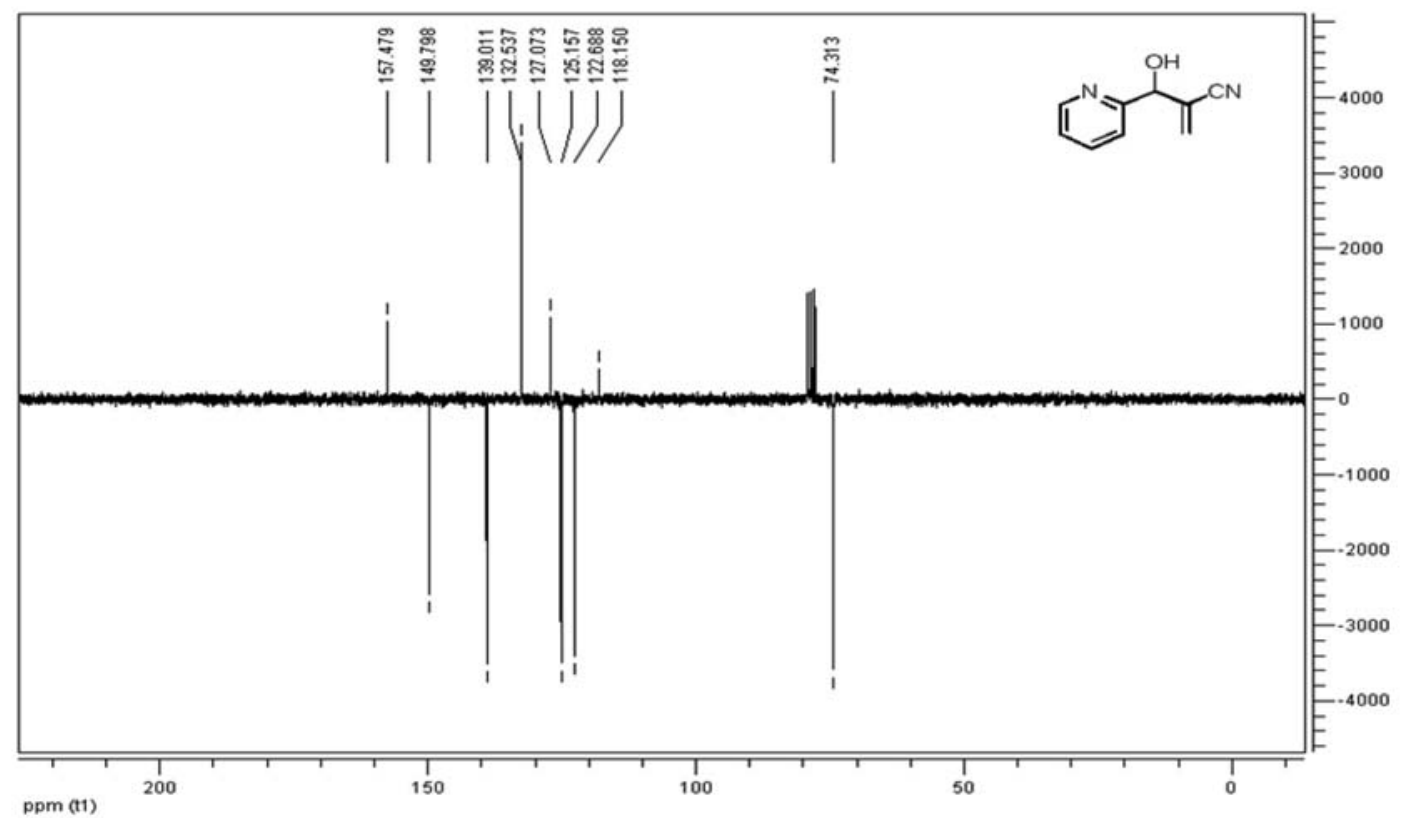

Figure S15. ${ }^{13} \mathrm{C}$ NMR $\left(\mathrm{CDCl}_{3}\right)$ spectrum of 2-[Hydroxy(pyridin-2-yl)methyl] acrylonitrile (3e). 


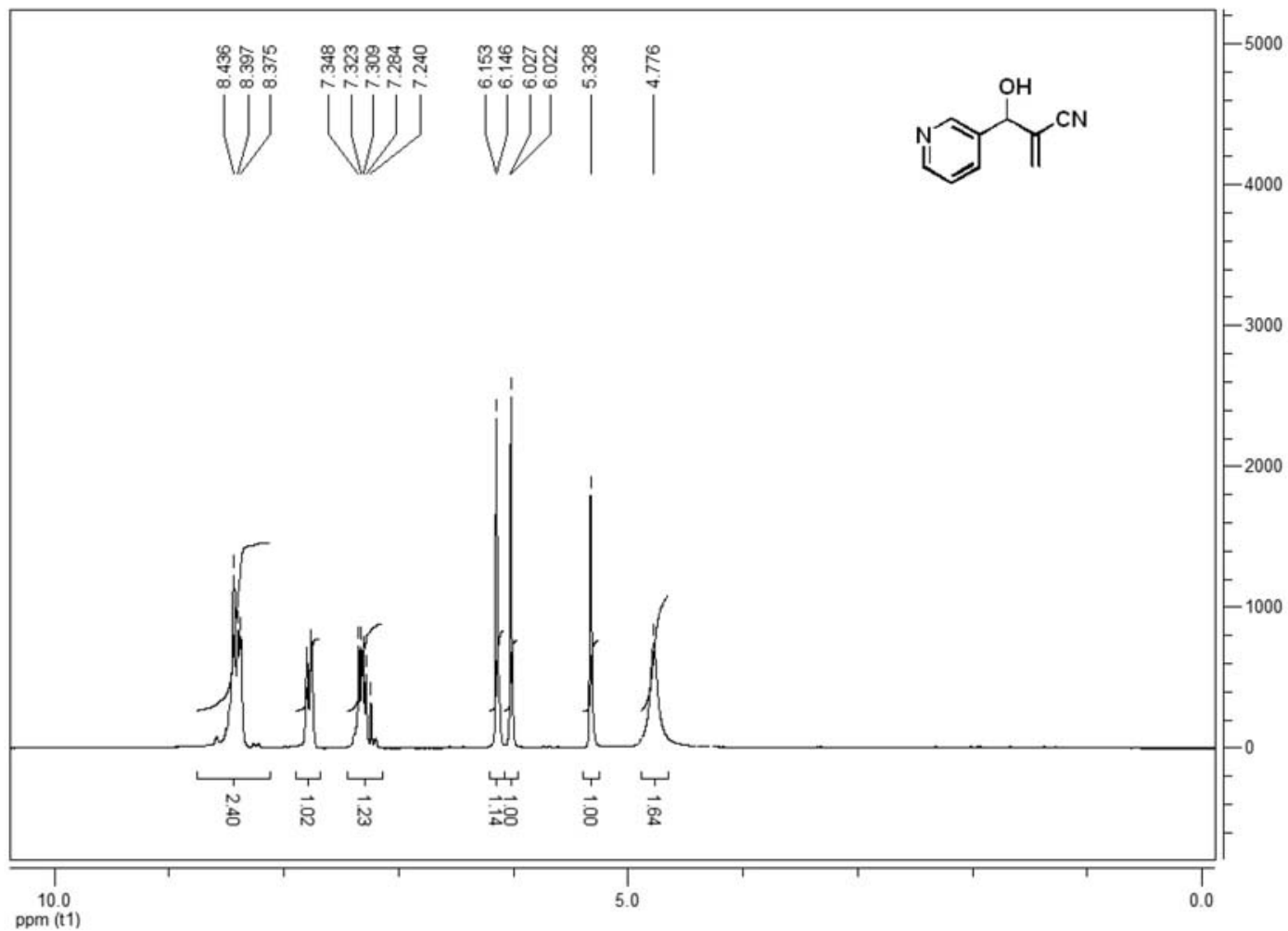

Figure S16. ${ }^{1} \mathrm{H}$ NMR $\left(\mathrm{CDCl}_{3}\right)$ spectrum of 2-[Hydroxy(pyridin-3-yl)methyl] acrylonitrile (3f).

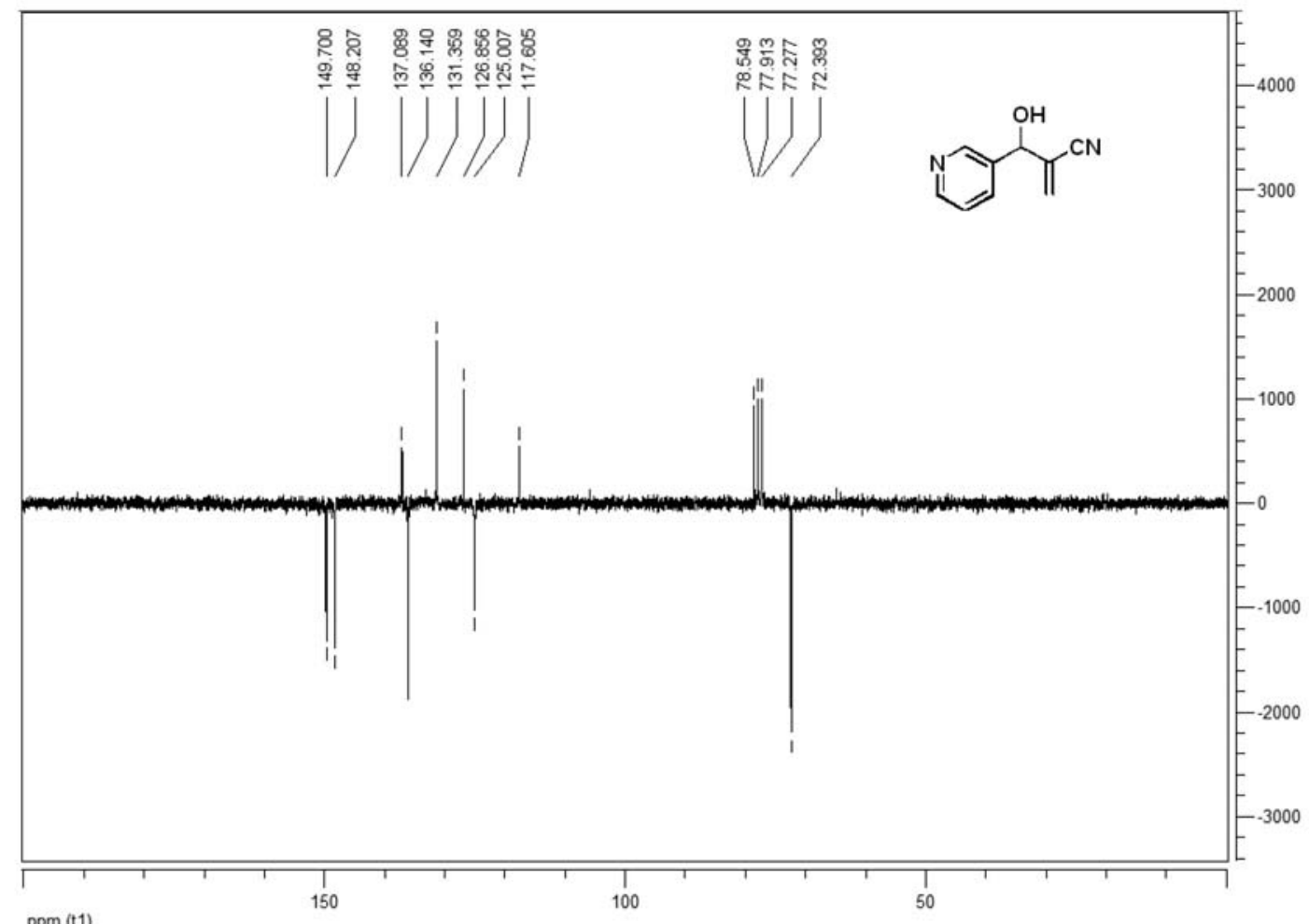

Figure S17. ${ }^{13} \mathrm{C}$ NMR $\left(\mathrm{CDCl}_{3}\right)$ spectrum of 2-[Hydroxy(pyridin-3-yl)methyl] acrylonitrile (3f). 


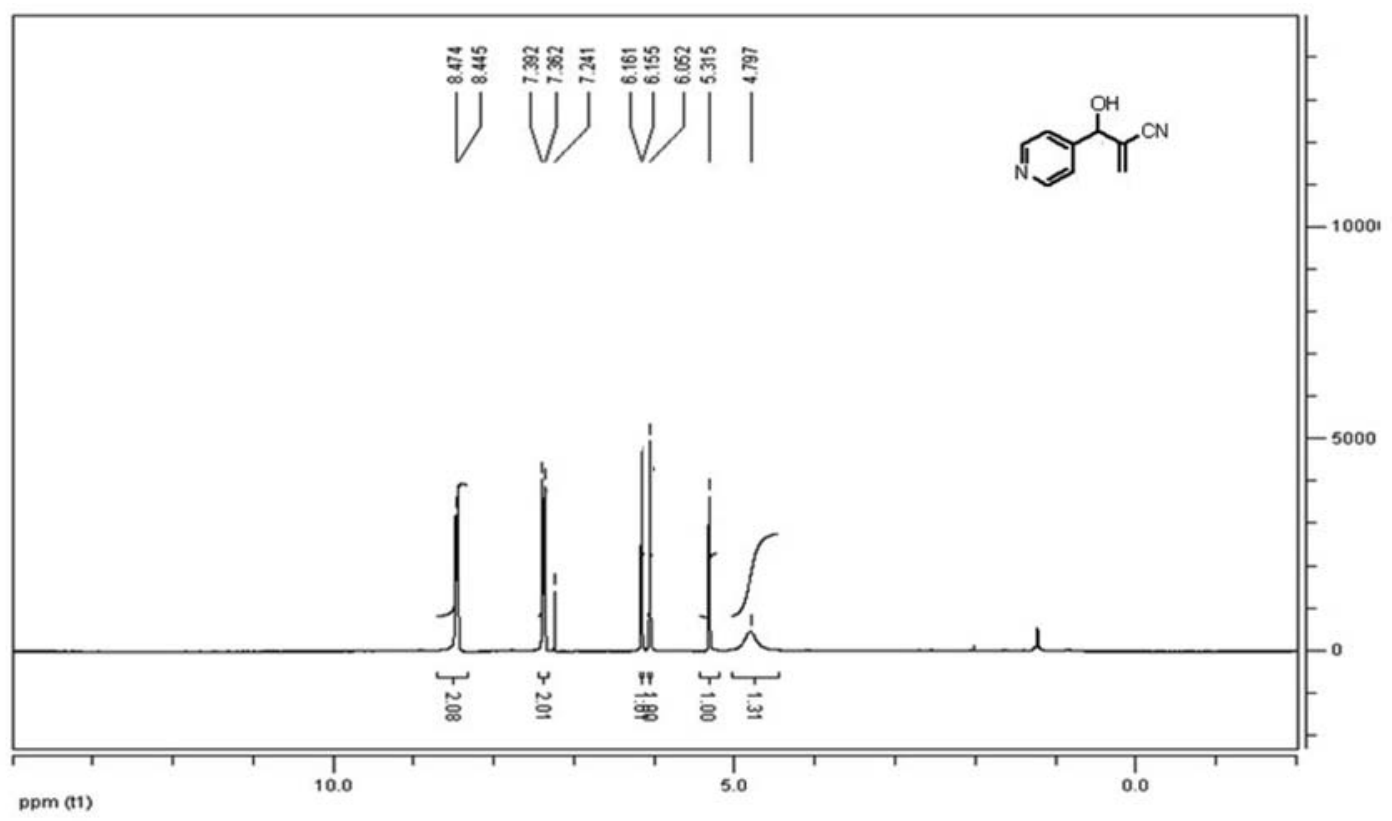

Figure S18. ${ }^{1} \mathrm{H}$ NMR $\left(\mathrm{CDCl}_{3}\right)$ spectrum of 2-[Hydroxy(pyridin-4-yl)methyl] acrylonitrile (3g).

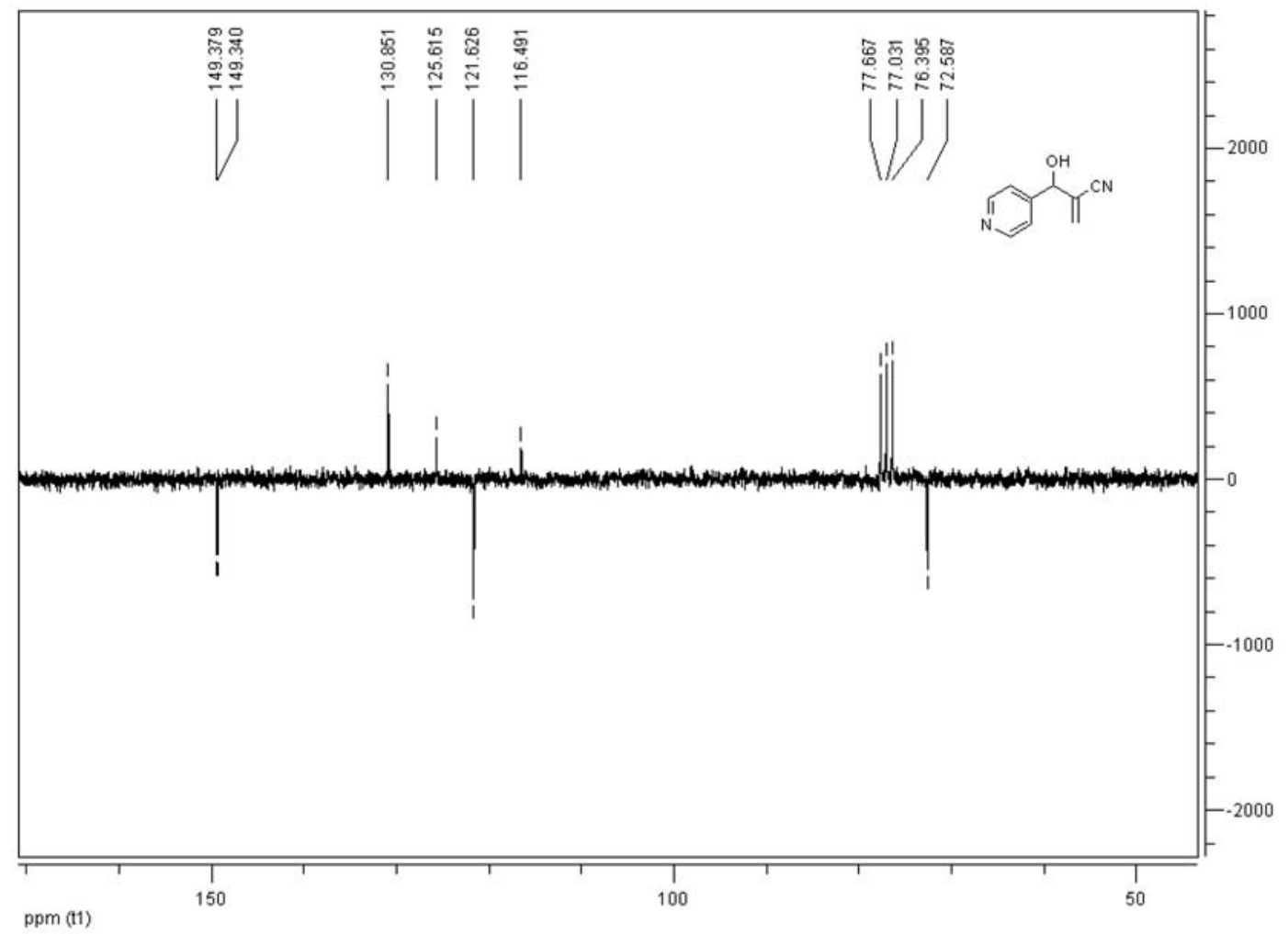

Figure S19. ${ }^{13} \mathrm{C}$ NMR $\left(\mathrm{CDCl}_{3}\right)$ spectrum of 2-[Hydroxy(pyridin-4-yl)methyl] acrylonitrile (3g). 


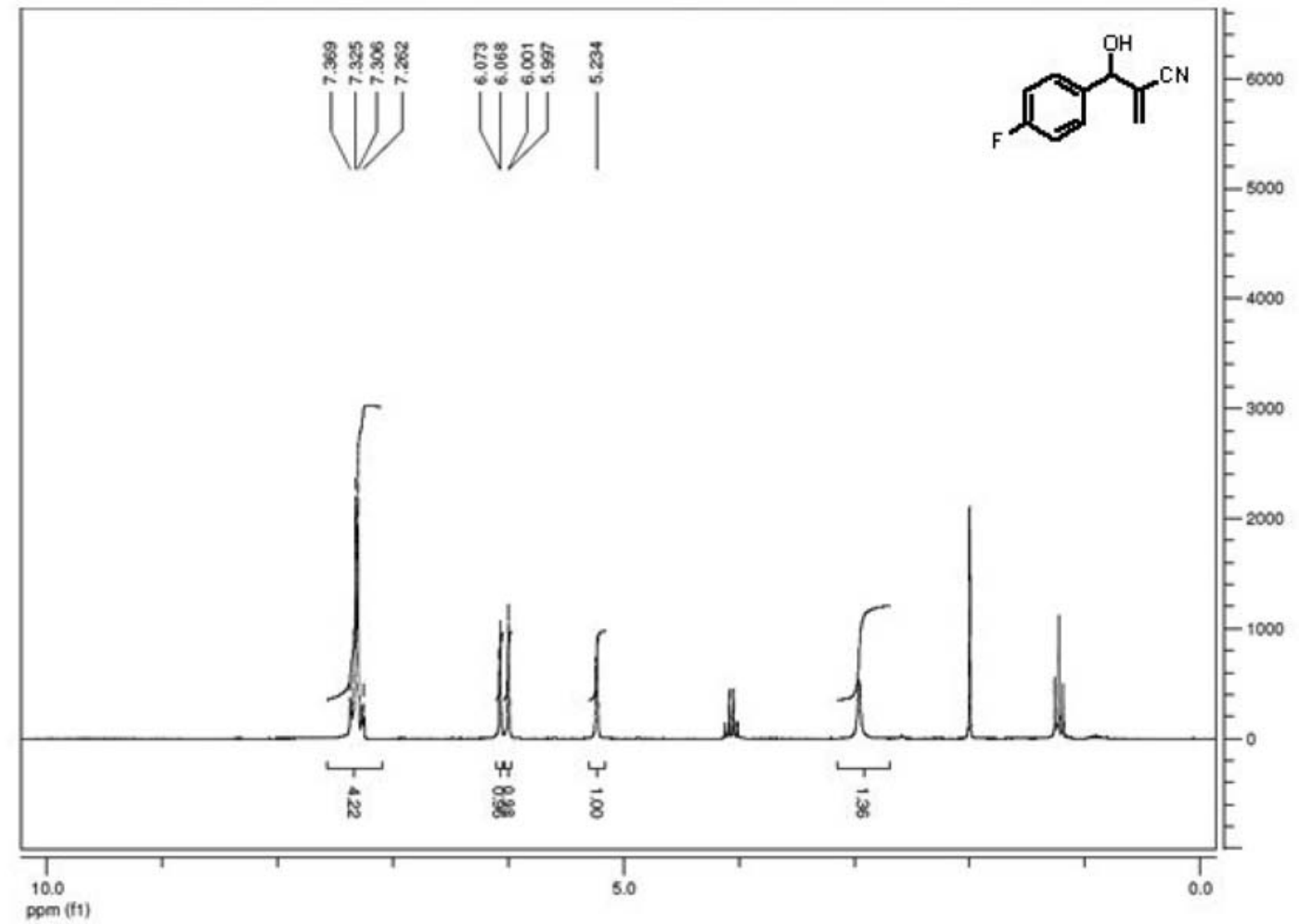

Figure S20. ${ }^{1} \mathrm{H}$ NMR $\left(\mathrm{CDCl}_{3}\right)$ spectrum of 2-[hydroxy (4-fluorphenyl) methyl] acrylonitrile (3h).

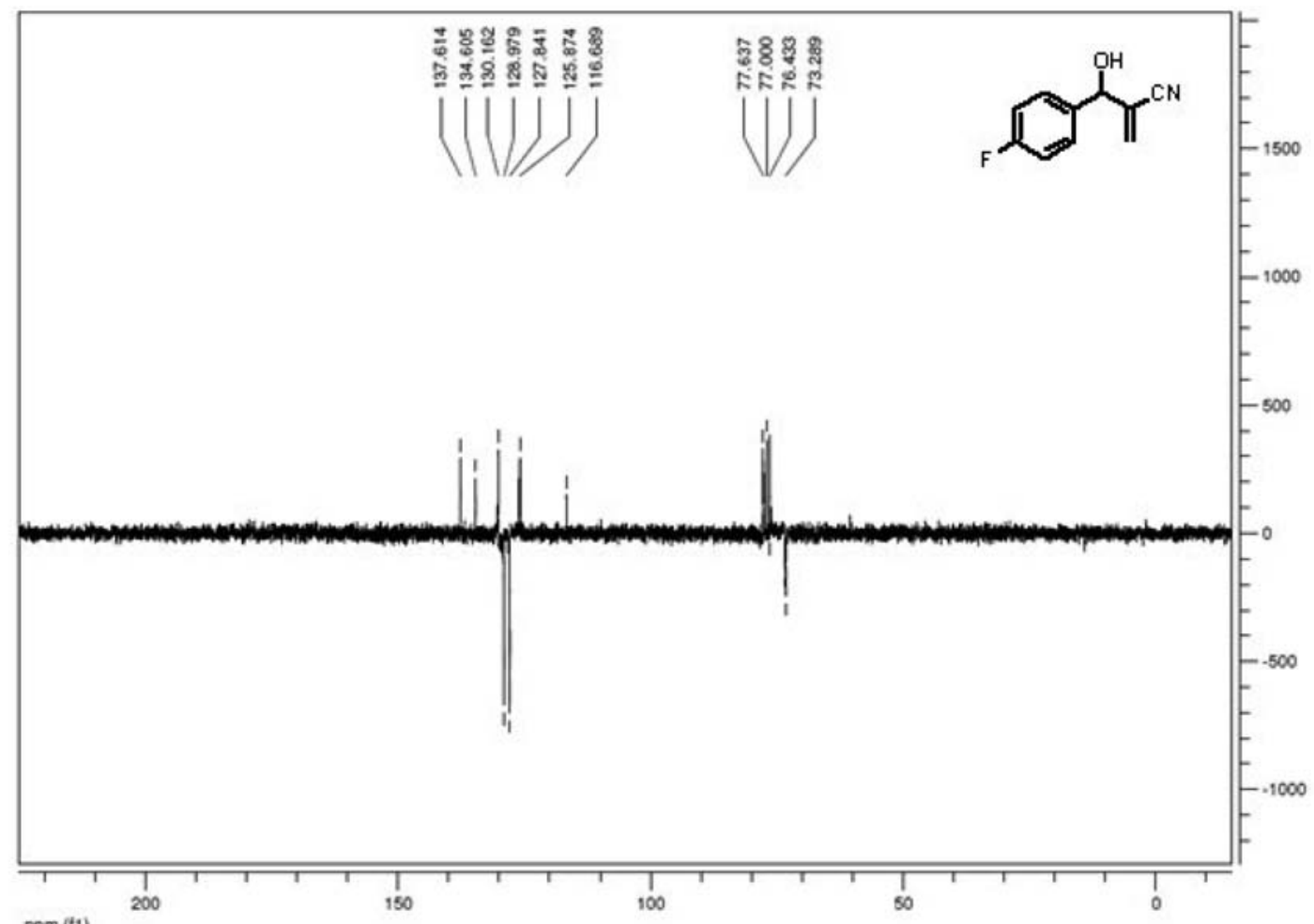

Figure S21. ${ }^{13} \mathrm{C}$ NMR $\left(\mathrm{CDCl}_{3}\right)$ spectrum of 2-[hydroxy (4-fluorphenyl) methyl] acrylonitrile (3h). 


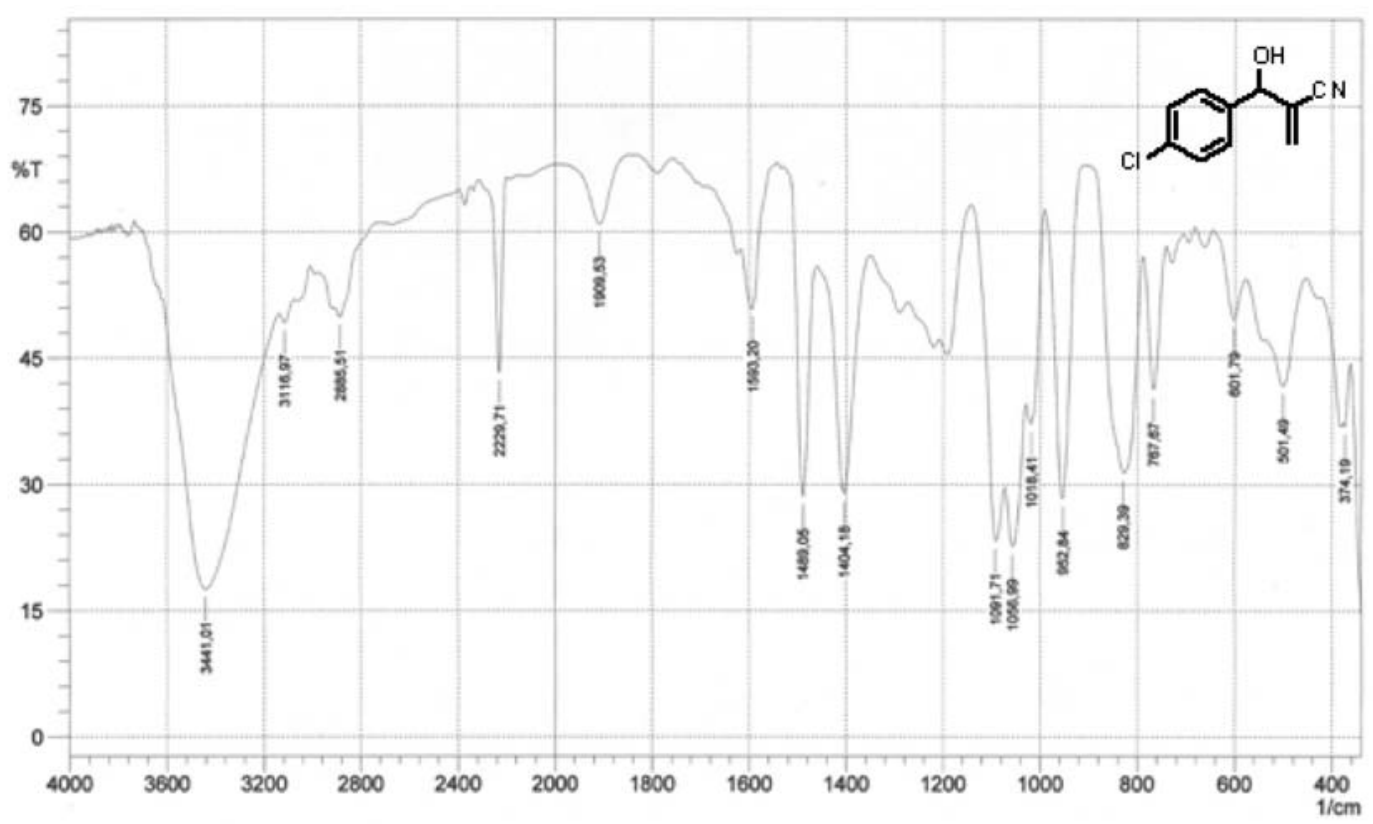

Figure S22. IR (KBr) of 2-[hydroxyl (4-chlorophenyl) methyl] acrylonitrile (3i).

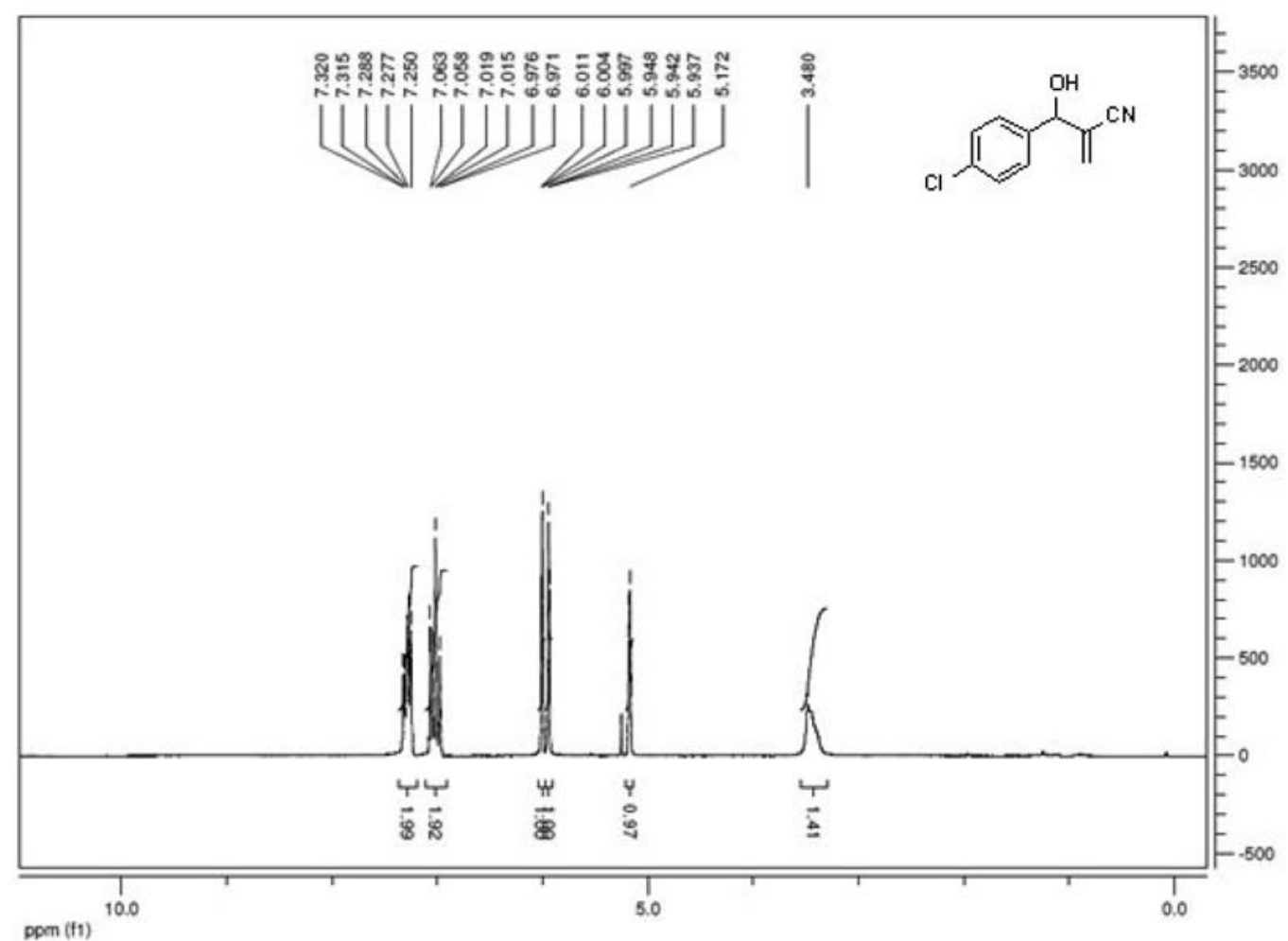

Figure S23. ${ }^{1} \mathrm{H}$ NMR $\left(\mathrm{CDCl}_{3}\right)$ spectrum of 2-[hydroxyl (4-chlorophenyl) methyl] acrylonitrile (3i). 


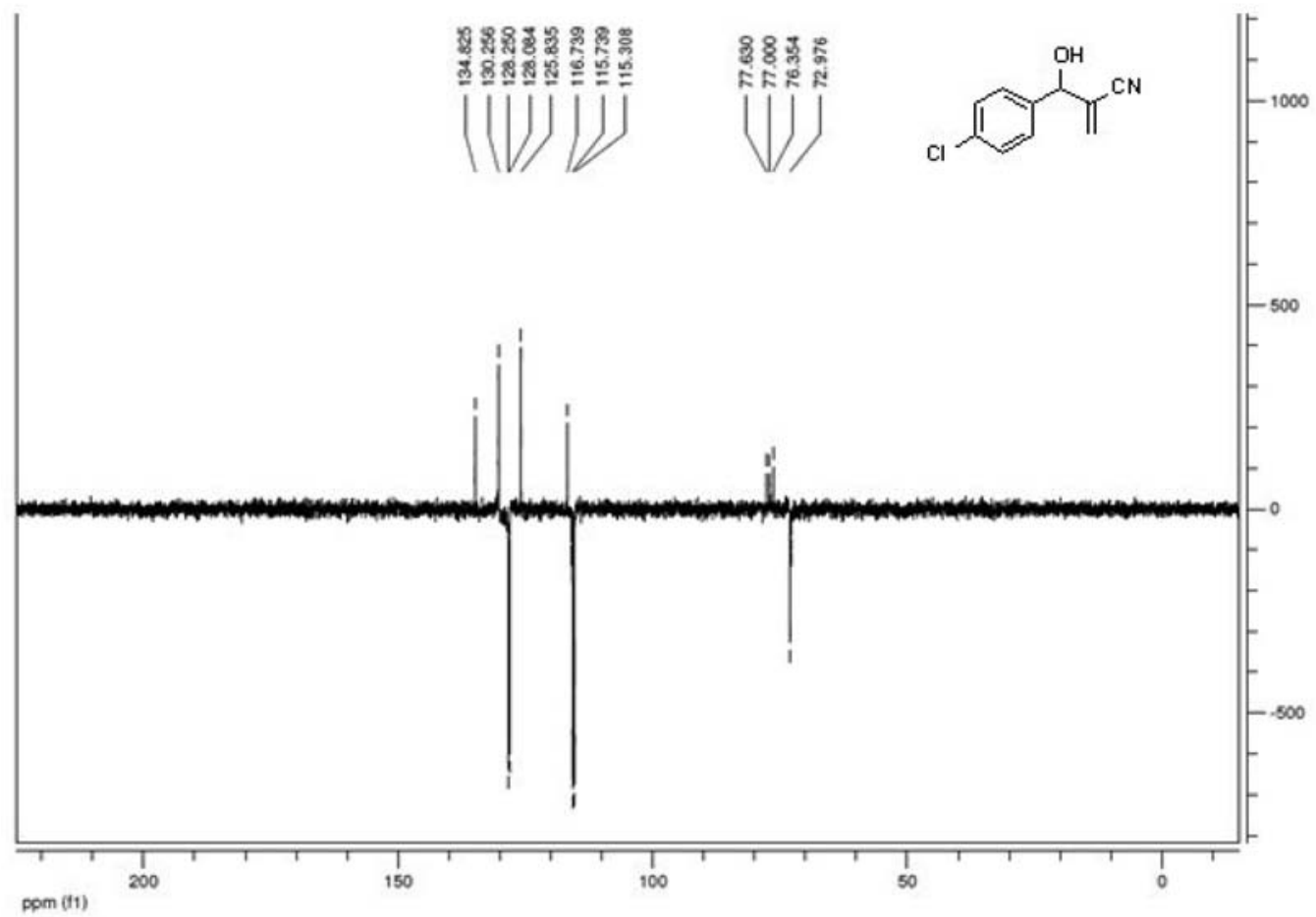

Figure S24. ${ }^{13} \mathrm{C}$ NMR $\left(\mathrm{CDCl}_{3}\right)$ spectrum of 2-[hydroxyl (4-chlorophenyl) methyl] acrylonitrile (3i).

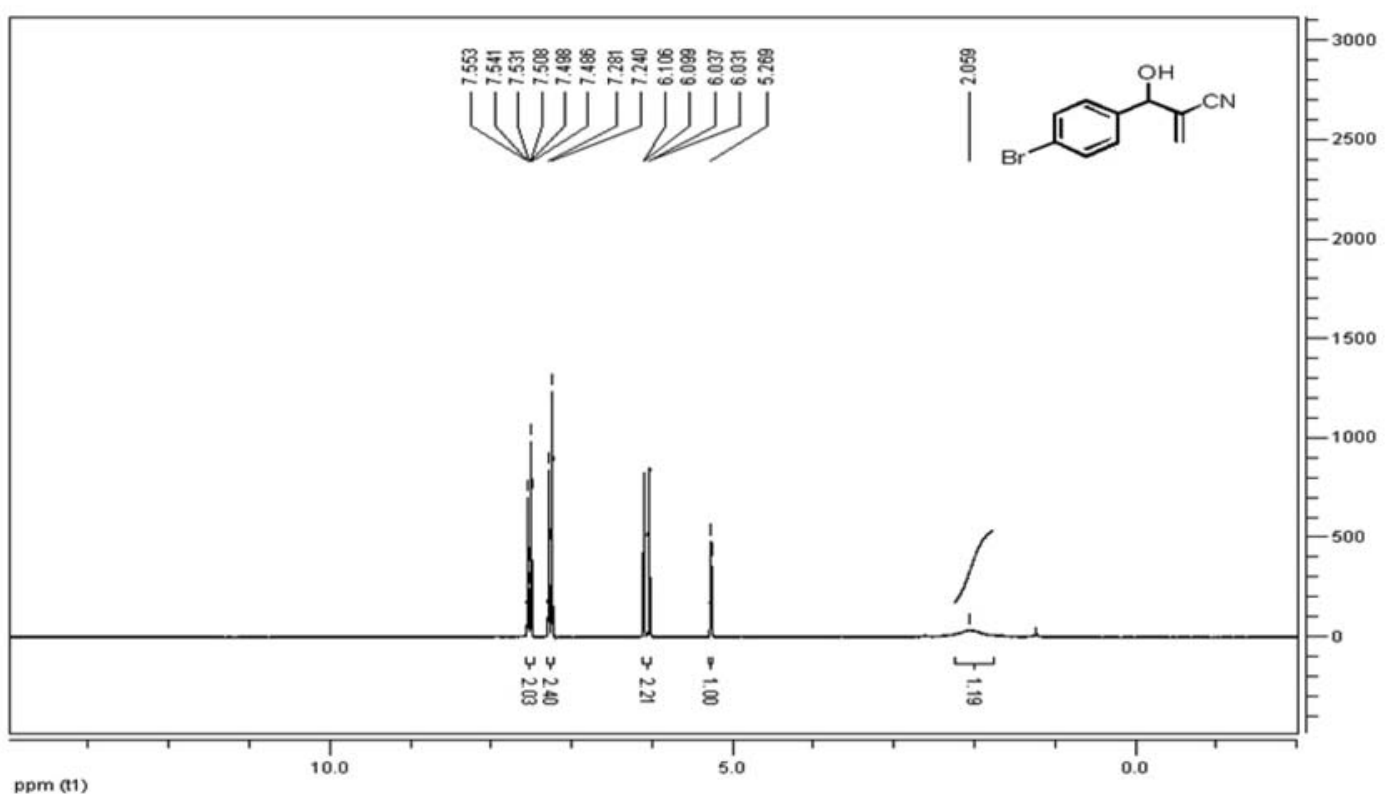

Figure S25. ${ }^{~} \mathrm{H}$ NMR $\left(\mathrm{CDCl}_{3}\right)$ spectrum of 2-[Hydroxy(4-bromophenyl)methyl] acrylonitrile (3j). 


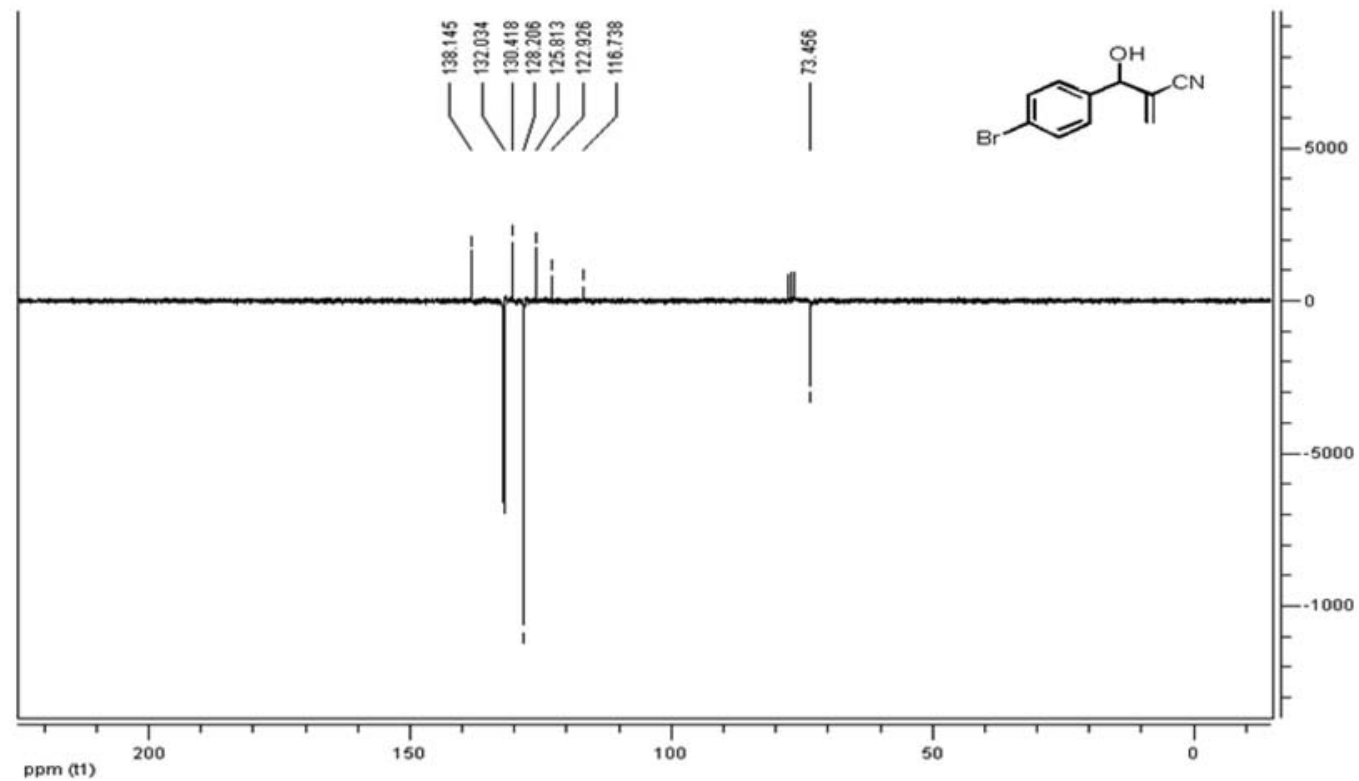

Figure S26. ${ }^{13} \mathrm{C} \mathrm{NMR}\left(\mathrm{CDCl}_{3}\right)$ spectrum of 2-[Hydroxy(4-bromophenyl)methyl] acrylonitrile (3j).

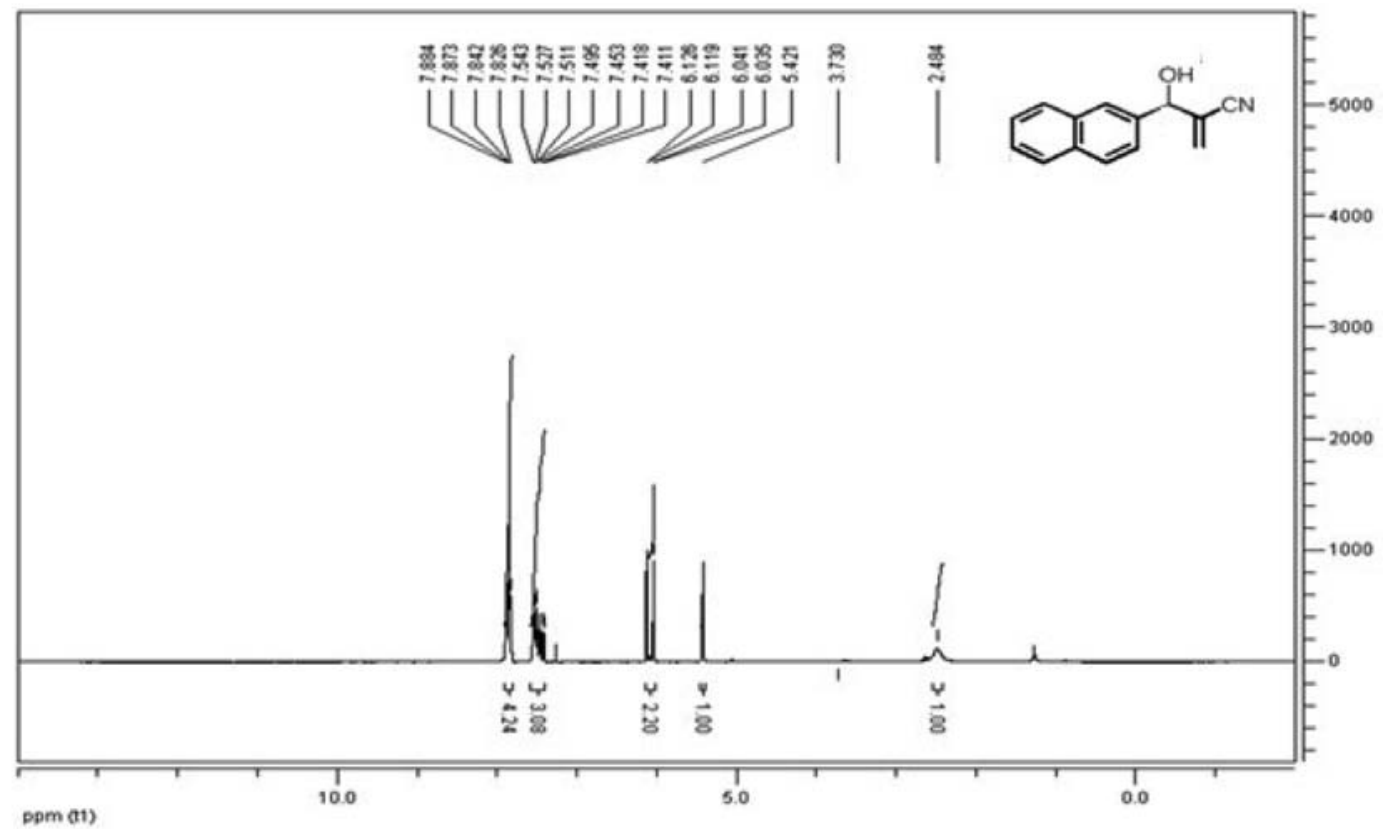

Figure S27. ${ }^{1} \mathrm{H}$ NMR $\left(\mathrm{CDCl}_{3}\right)$ spectrum of 2-[Hydroxy(naphth-2-yl)methyl] acrylonitrile (3k). 


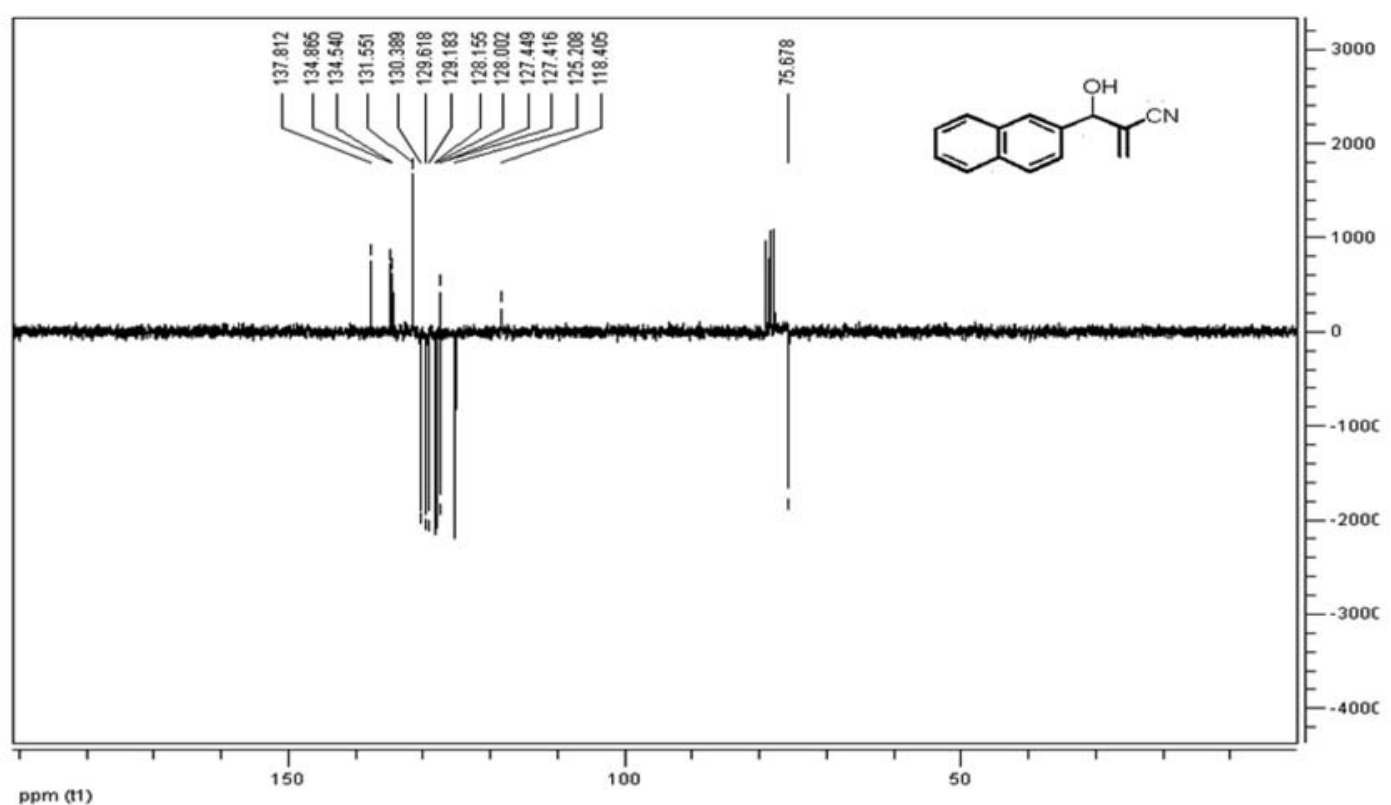

Figure S28. ${ }^{13} \mathrm{C}$ NMR $\left(\mathrm{CDCl}_{3}\right)$ spectrum of 2-[Hydroxy(naphth-2-yl)methyl] acrylonitrile (3k).

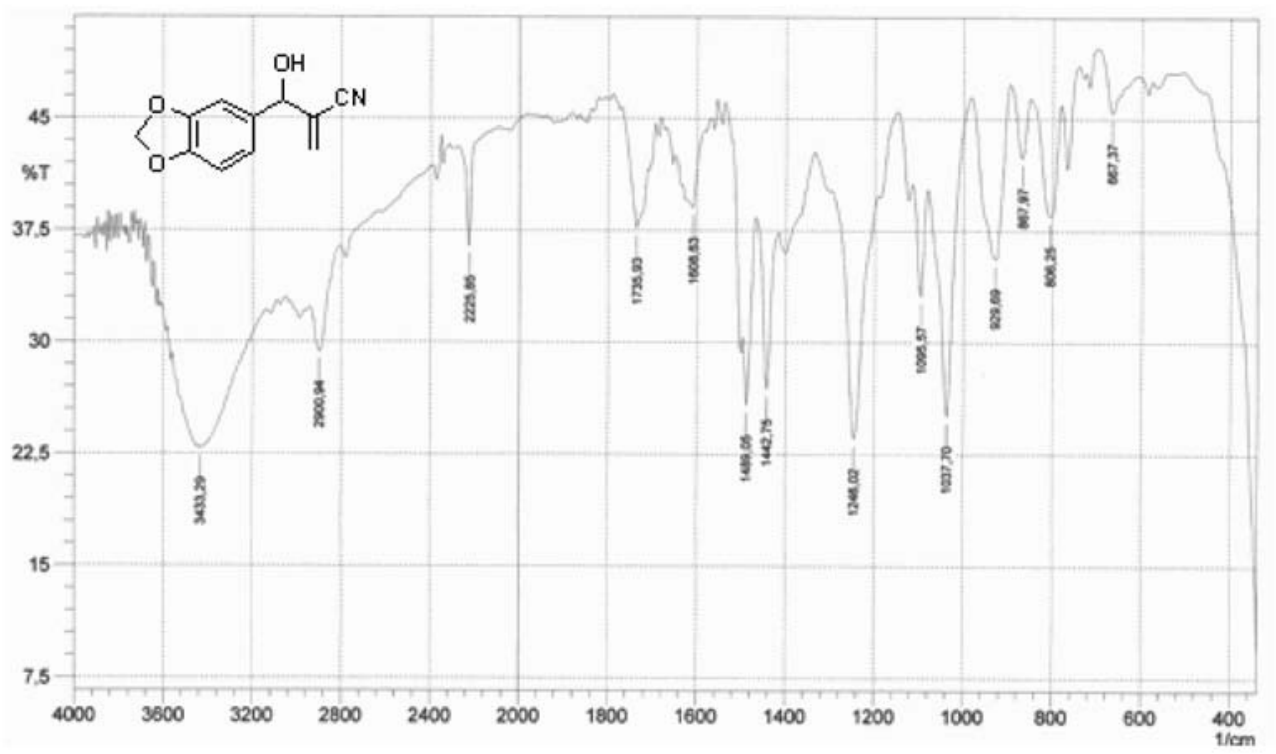

Figure S29. IR (KBr) of 2-[hydroxyl (1,3-dioxolephenyl) methyl] acrylonitrile (31). 


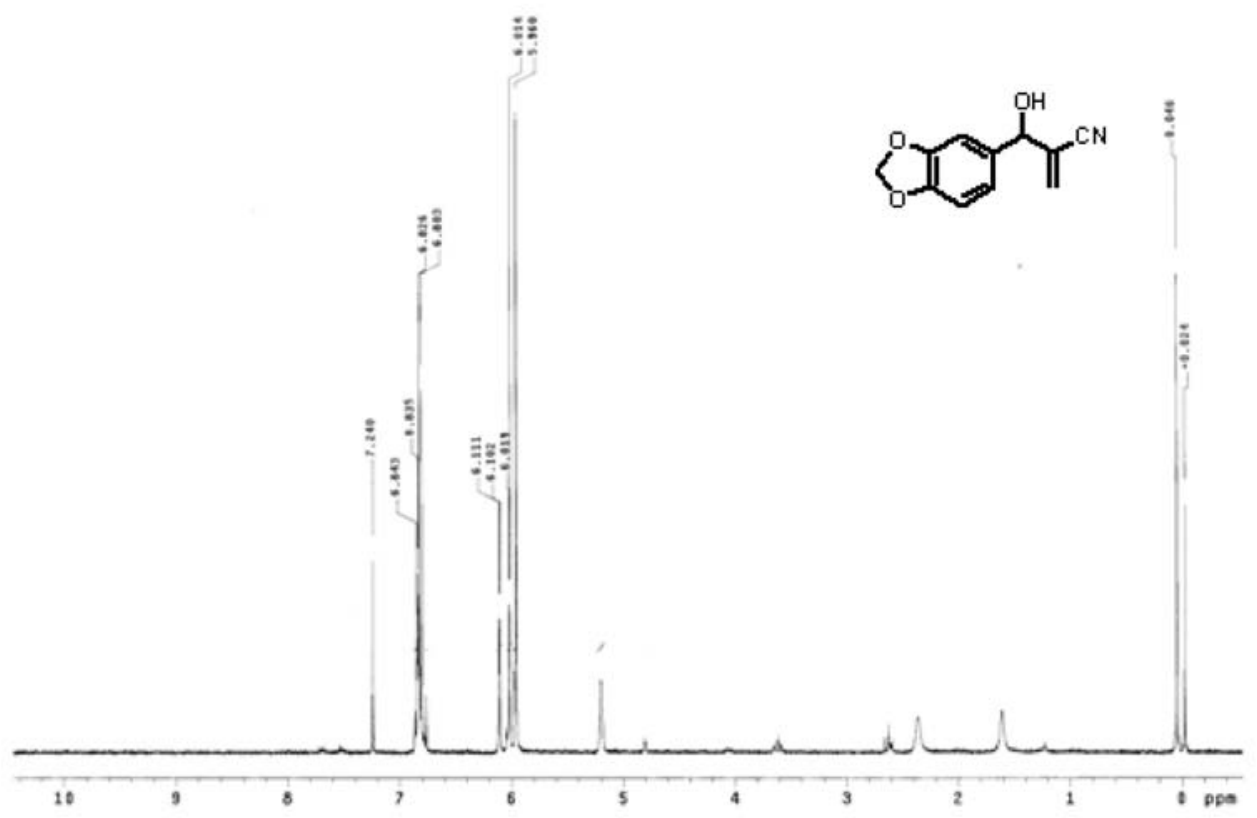

Figure S30. ${ }^{1} \mathrm{H}$ NMR $\left(\mathrm{CDCl}_{3}\right)$ spectrum of 2-[hydroxyl (1,3-dioxolephenyl) methyl] acrylonitrile (3l).

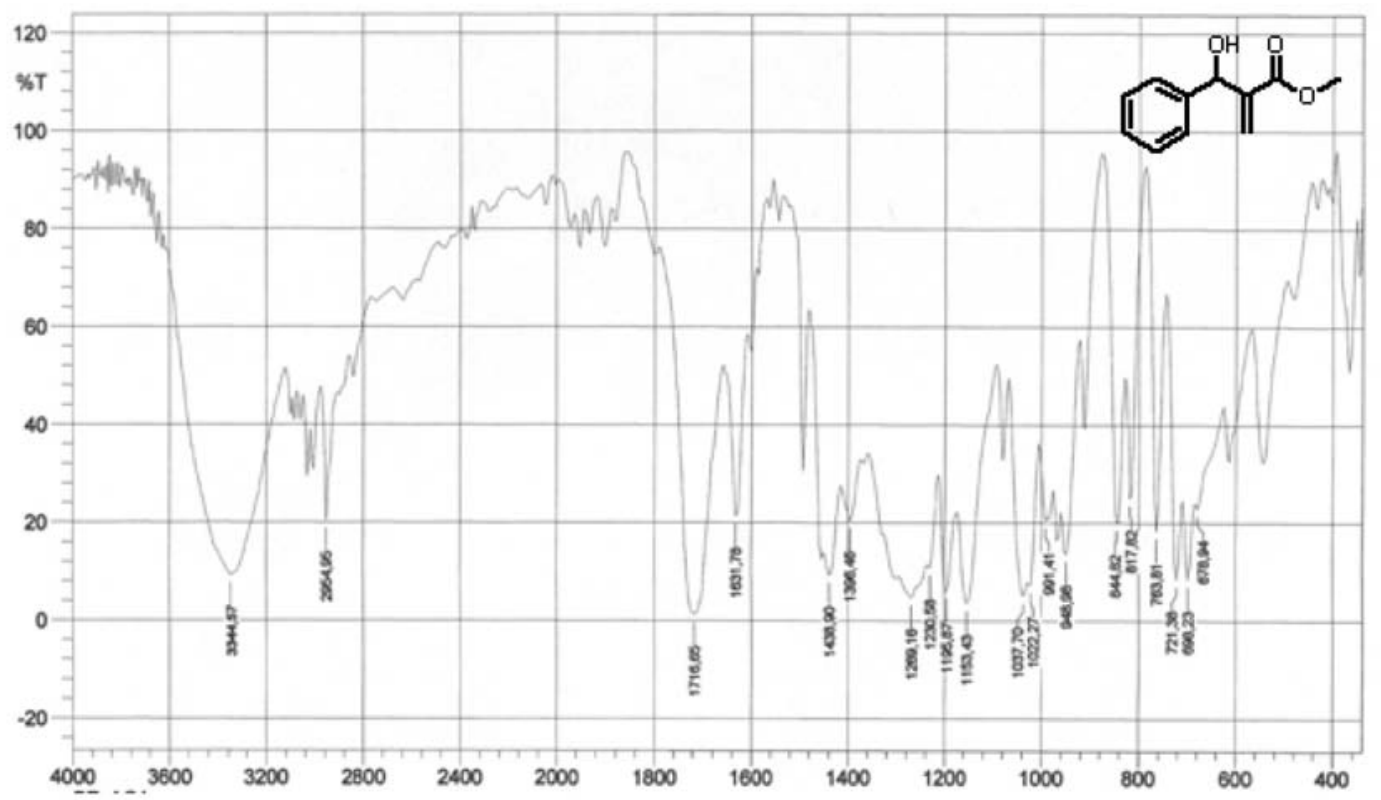

Figure S31. IR (KBr) of Methyl 2-[(hydroxylphenyl)methyl]acrylate (6a). 


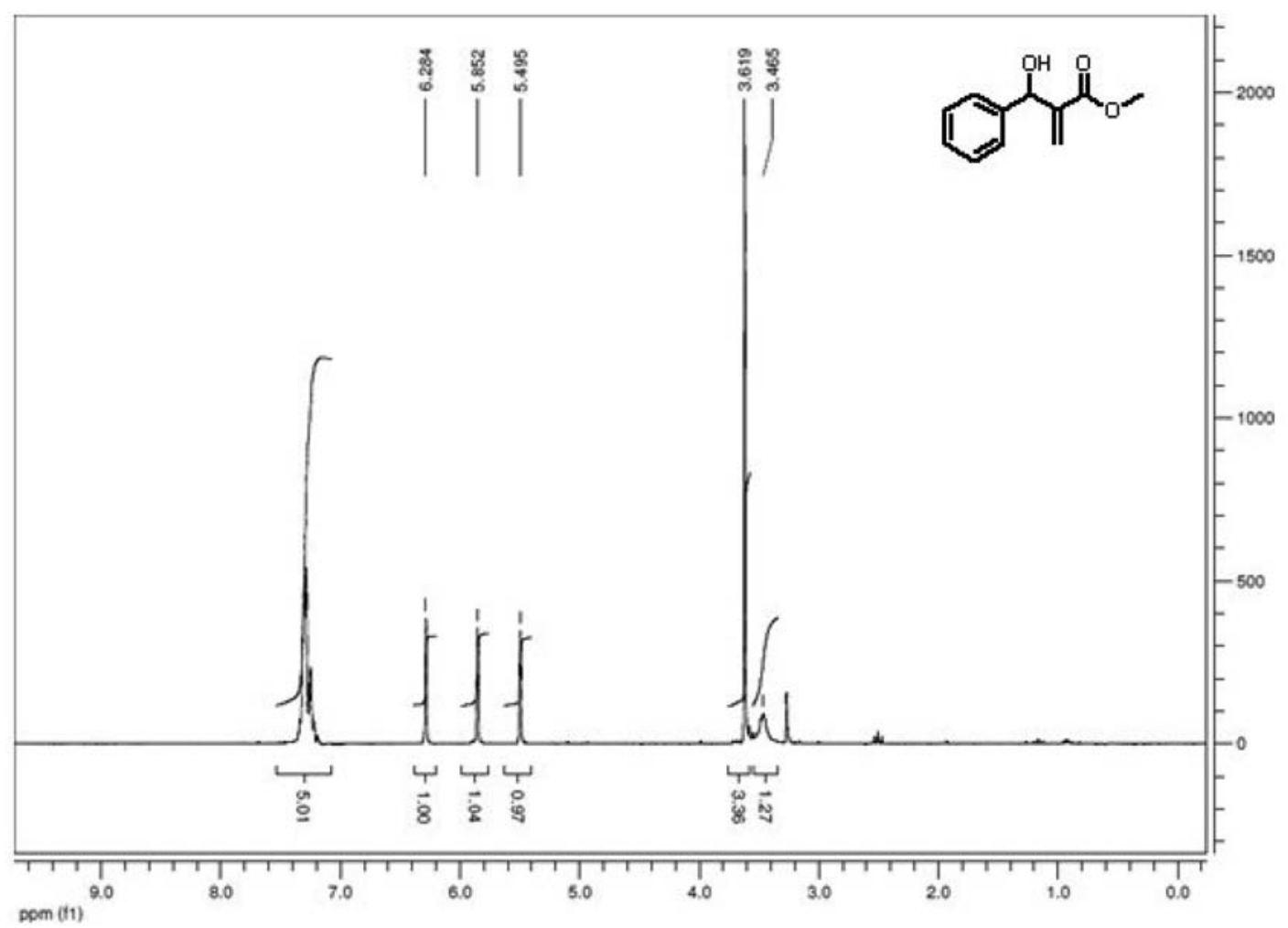

Figure S32. ${ }^{1} \mathrm{H}$ NMR $\left(\mathrm{CDCl}_{3}\right)$ spectrum of Methyl 2-[(hydroxylphenyl)methyl]acrylate (6a).

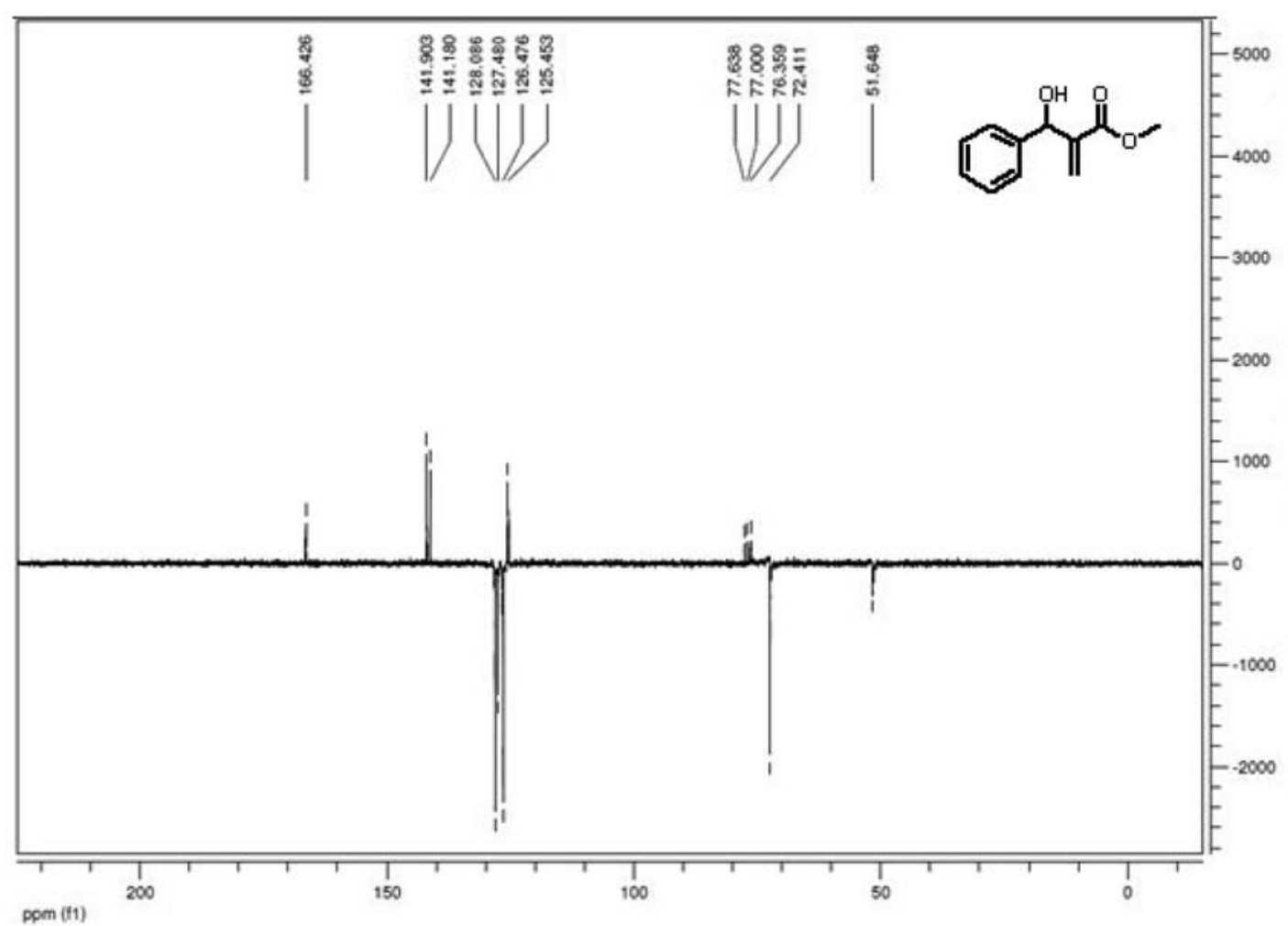

Figure S33. ${ }^{13} \mathrm{C}$ NMR $\left(\mathrm{CDCl}_{3}\right)$ spectrum of Methyl 2-[(hydroxylphenyl)methyl]acrylate (6a). 


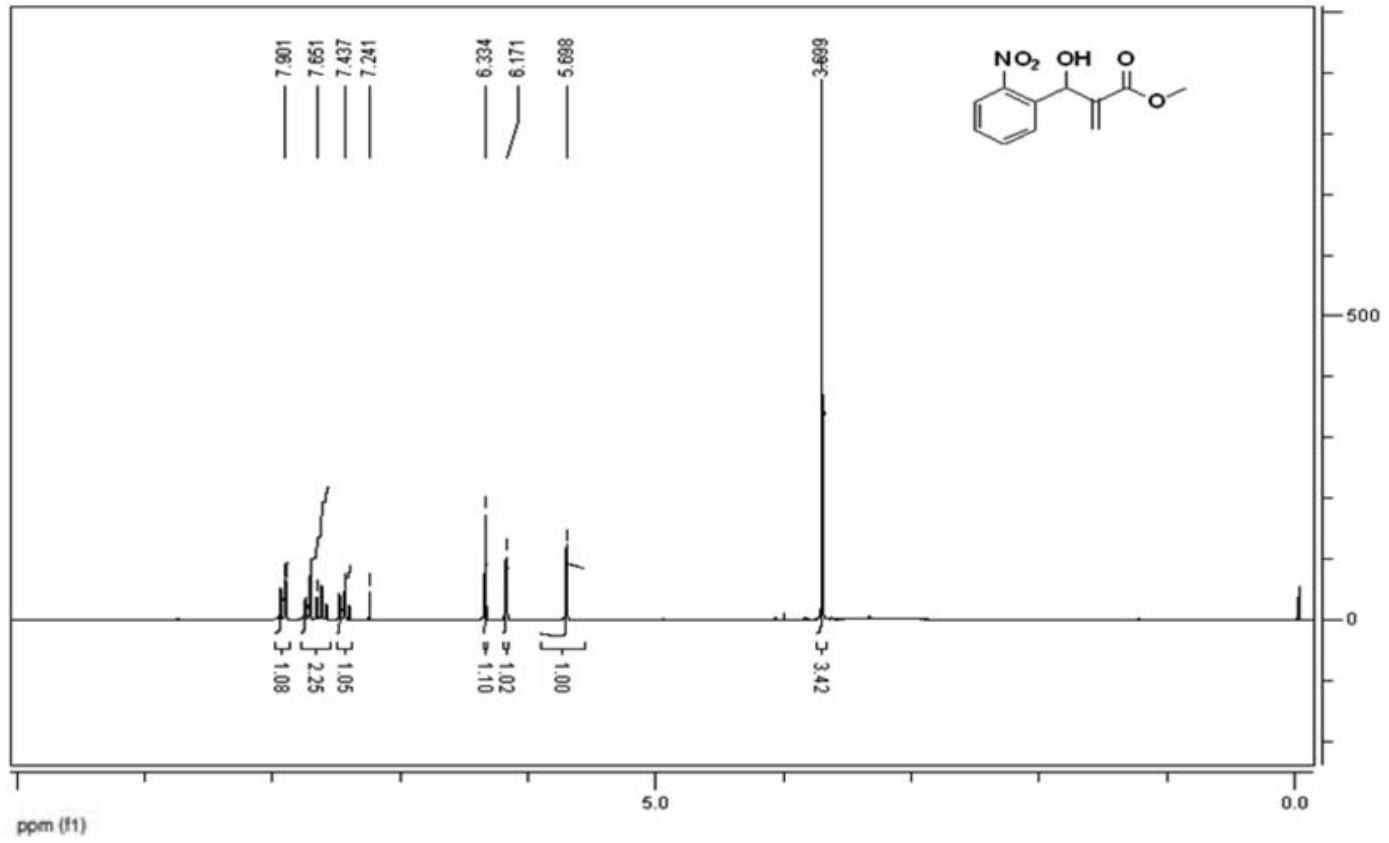

Figure S34. ${ }^{1} \mathrm{H}$ NMR $\left(\mathrm{CDCl}_{3}\right)$ spectrum of Methyl 2-[hydroxyl (2-nitrophenyl)methyl] acrylate (6b).

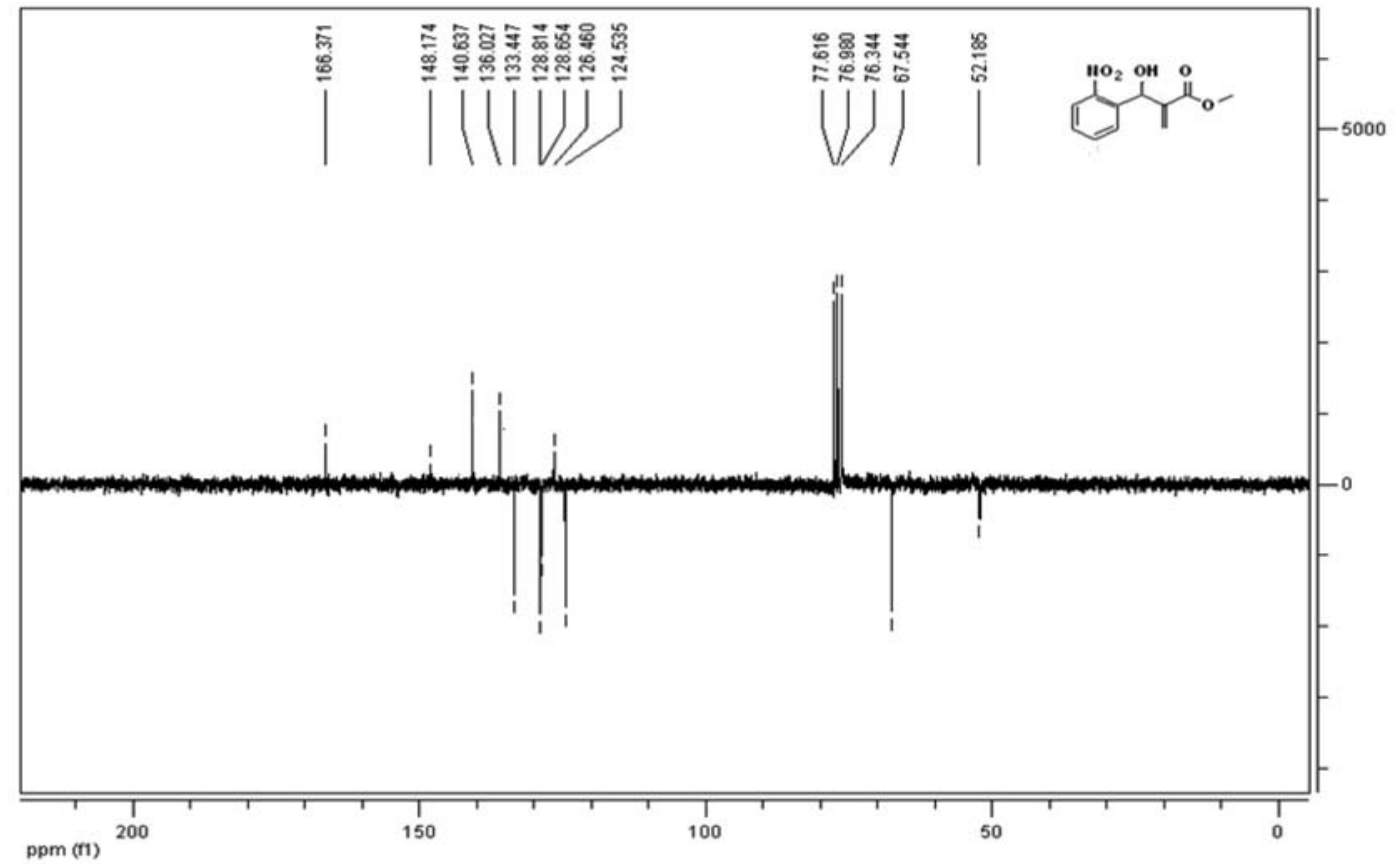

Figure S35. ${ }^{13} \mathrm{C}$ NMR $\left(\mathrm{CDCl}_{3}\right)$ spectrum of Methyl 2-[hydroxyl (2-nitrophenyl)methyl] acrylate (6b). 


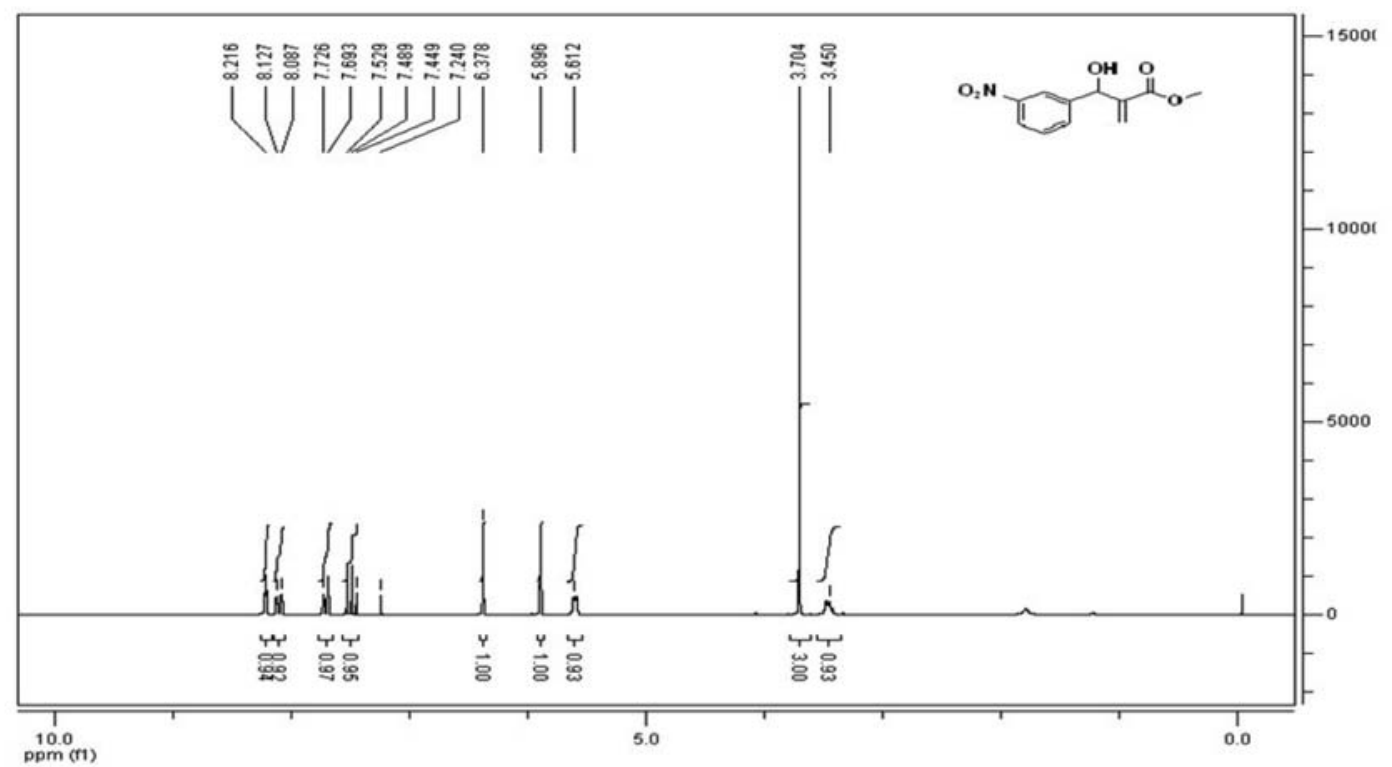

Figure S36. ${ }^{1} \mathrm{H}$ NMR $\left(\mathrm{CDCl}_{3}\right)$ spectrum of Methyl 2-[hydroxyl(3-nitrophenyl)methyl] acrylate (6c).

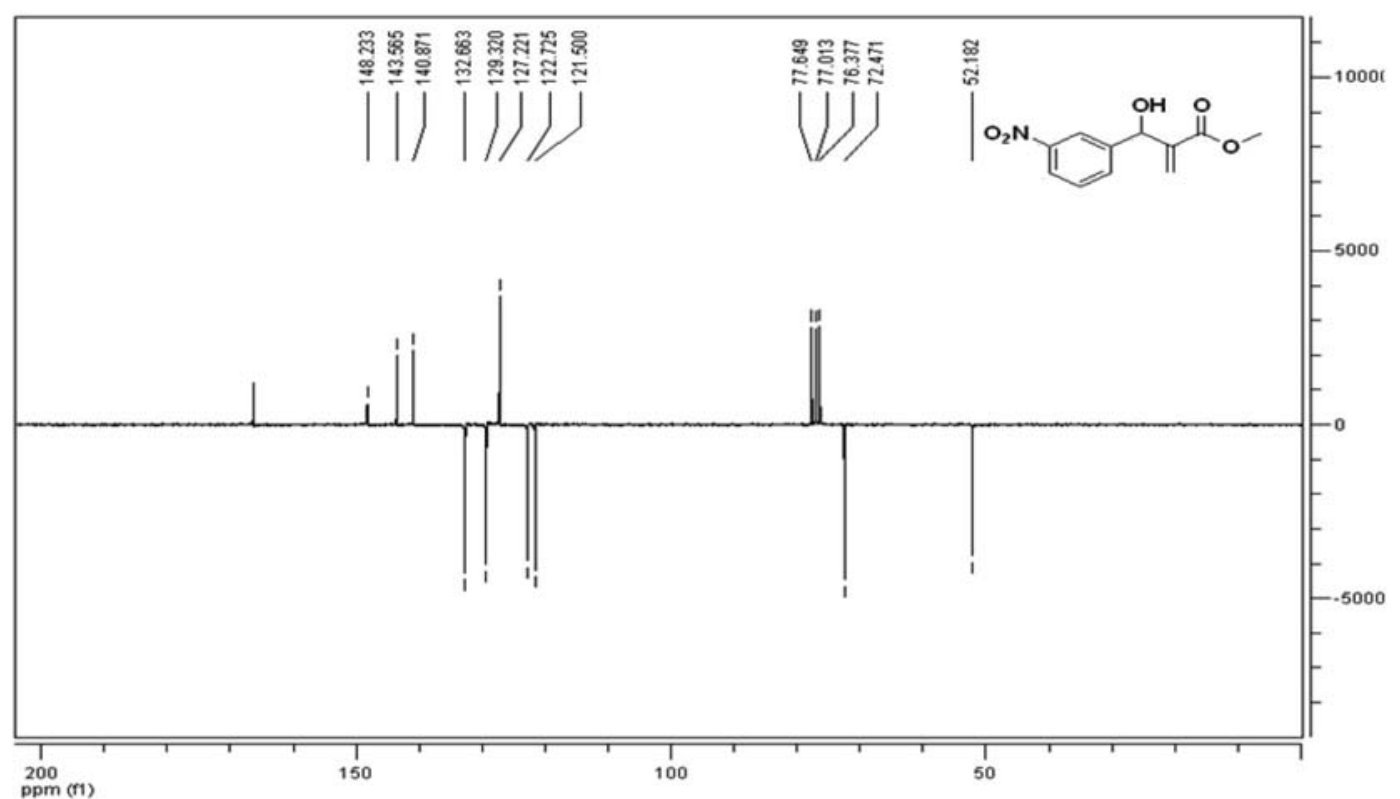

Figure S37. ${ }^{13} \mathrm{C}$ NMR $\left(\mathrm{CDCl}_{3}\right)$ spectrum of Methyl 2-[hydroxyl(3-nitrophenyl)methyl] acrylate (6c). 


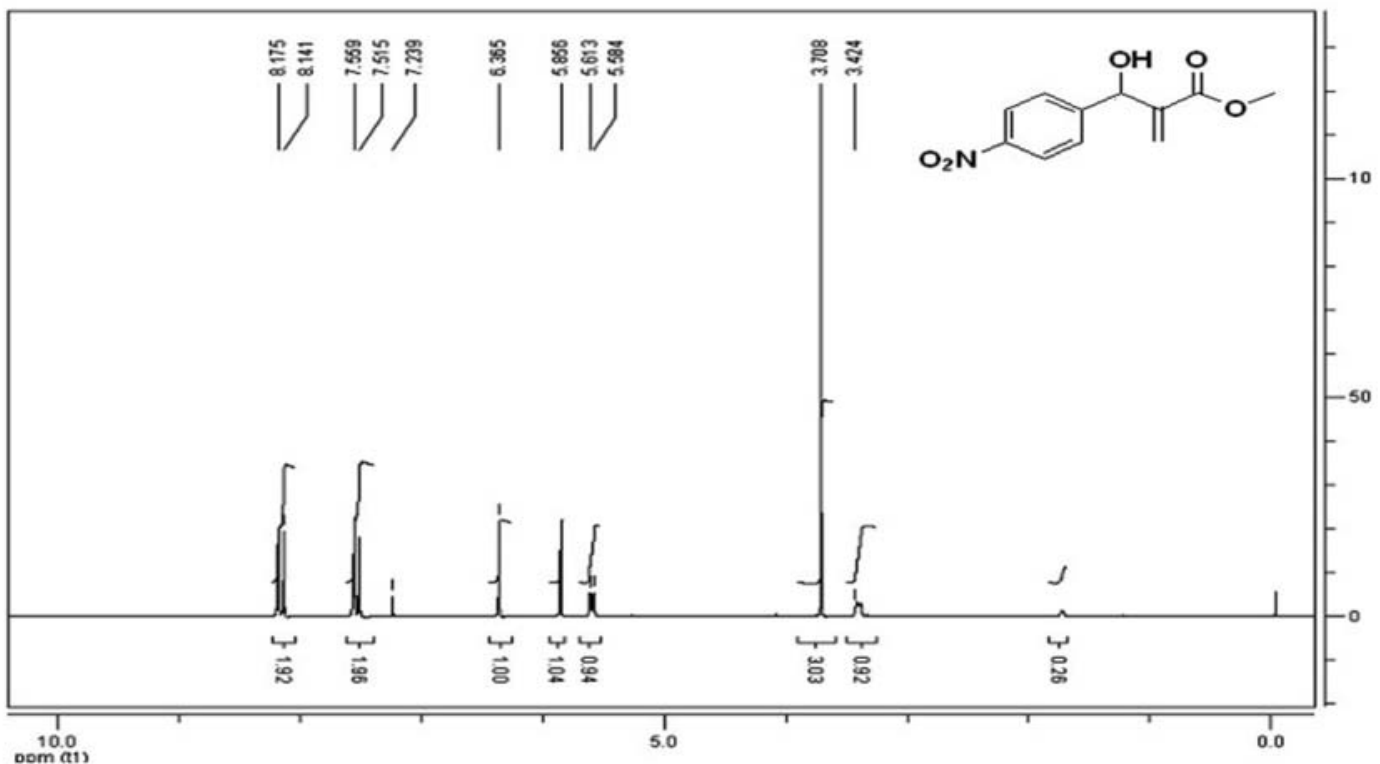

Figure S38. ${ }^{1} \mathrm{H}$ NMR $\left(\mathrm{CDCl}_{3}\right)$ spectrum of Methyl 2-[hydroxyl(4-nitrophenyl)methyl]acrylate (6d).

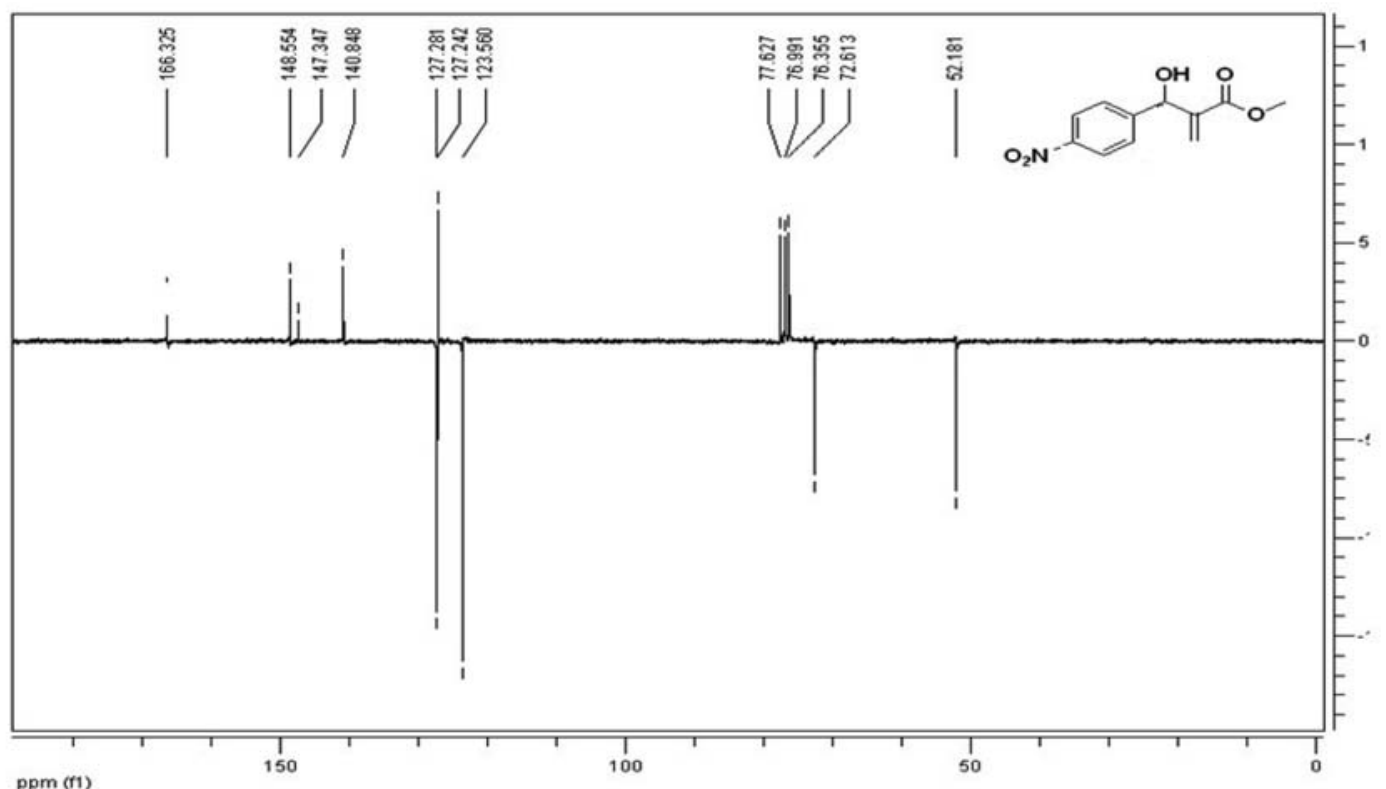

Figure S39. ${ }^{13} \mathrm{C}$ NMR $\left(\mathrm{CDCl}_{3}\right)$ spectrum of Methyl 2-[hydroxyl(4-nitrophenyl)methyl]acrylate (6d). 


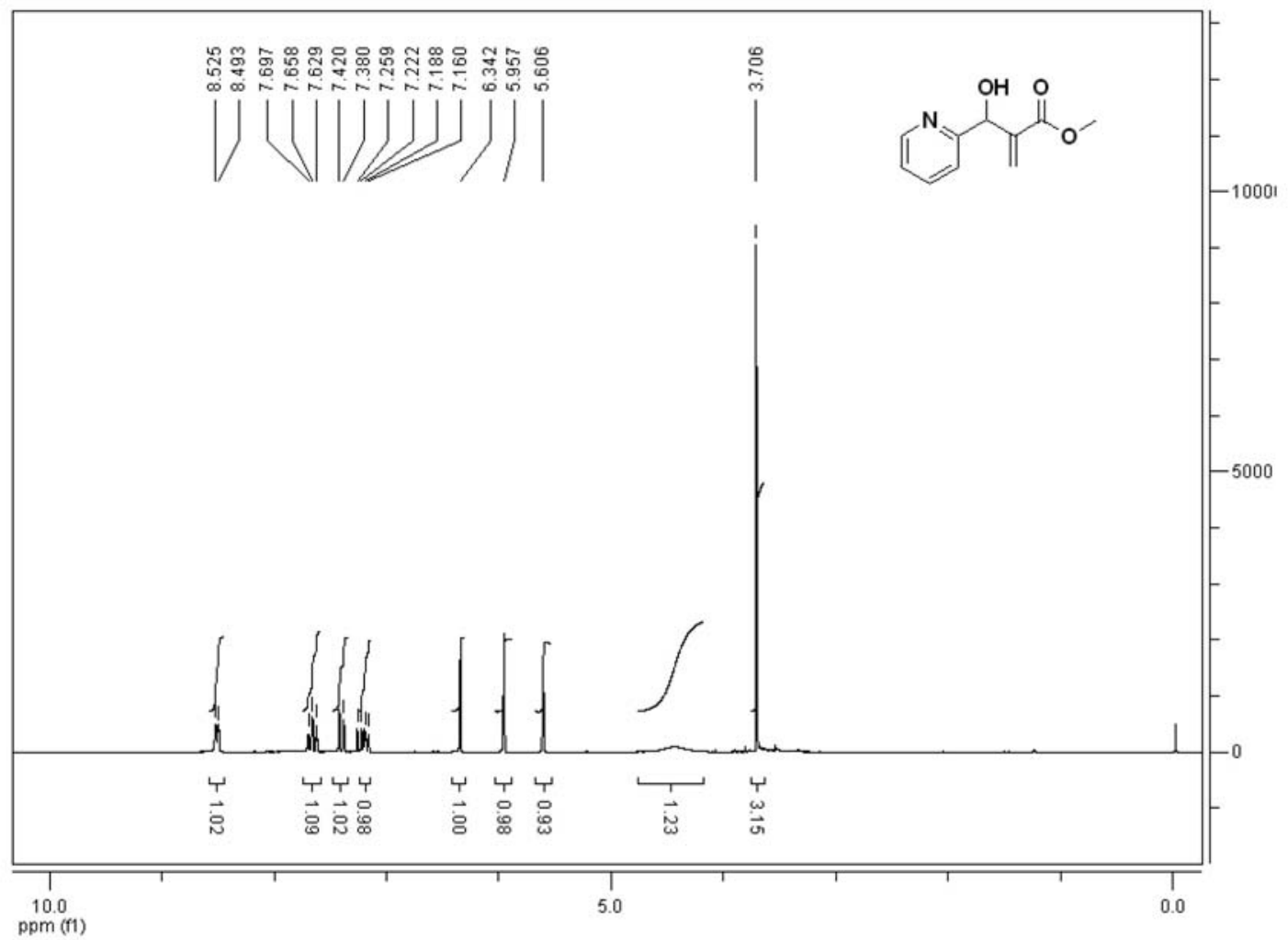

Figure S40. ${ }^{1} \mathrm{H}$ NMR $\left(\mathrm{CDCl}_{3}\right)$ spectrum of Methyl 2-[hydroxyl(2-pyridinyl)methyl] acrylate (6e).

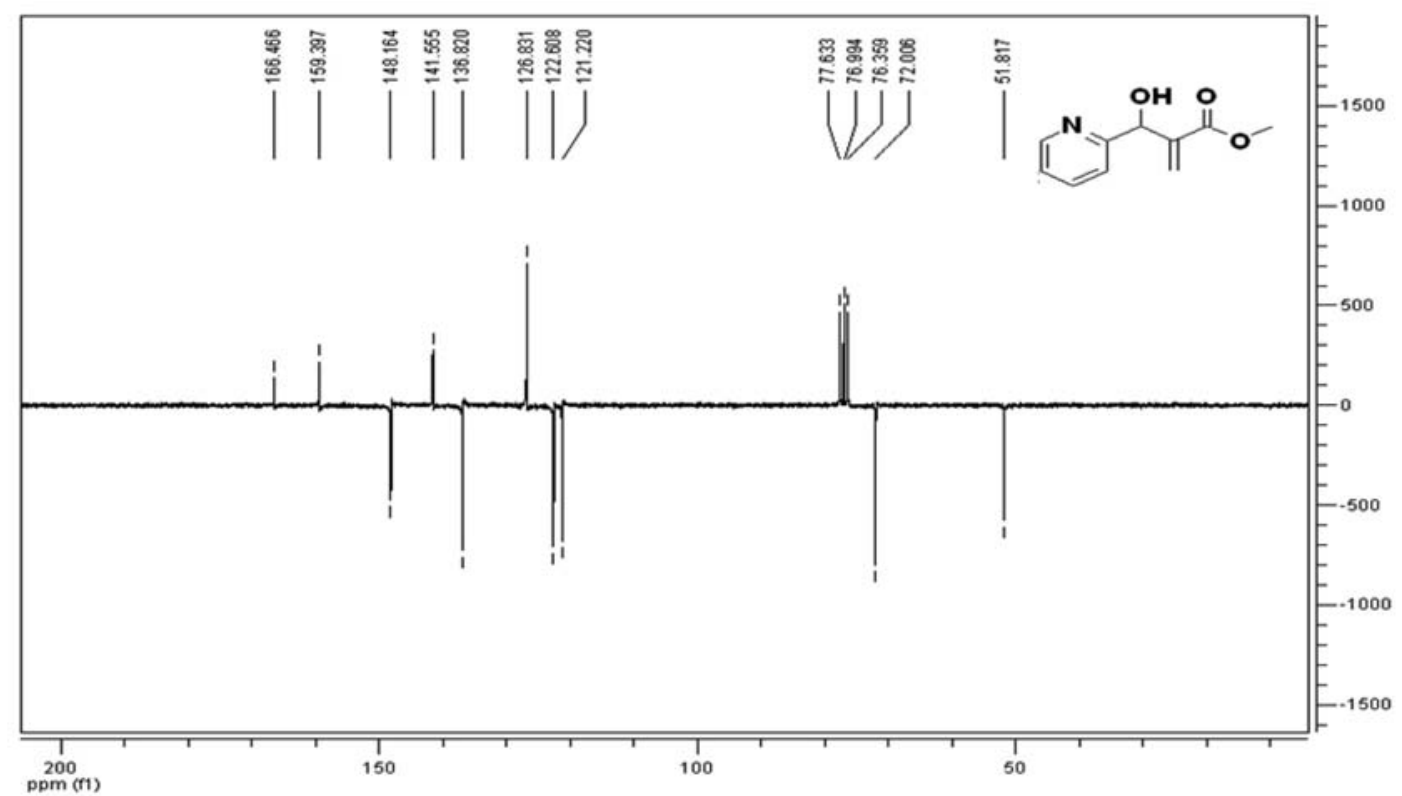

Figure S41. ${ }^{13} \mathrm{C}$ NMR $\left(\mathrm{CDCl}_{3}\right)$ spectrum of Methyl 2-[hydroxyl(2-pyridinyl)methyl] acrylate (6e). 


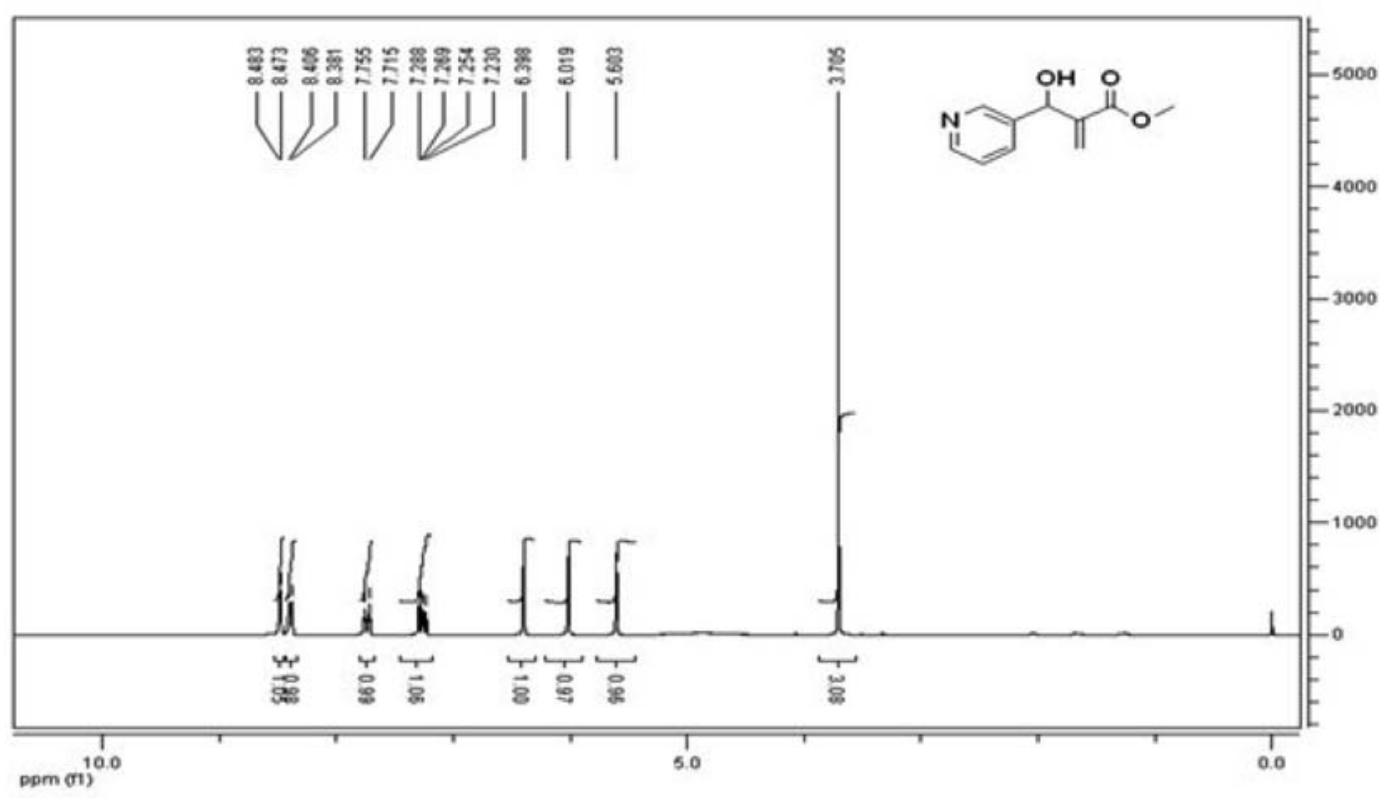

Figure S42. ${ }^{1} \mathrm{H}$ NMR $\left(\mathrm{CDCl}_{3}\right)$ spectrum of Methyl 2-[hydroxyl(3-pyridinyl)methyl] acrylate (6f).

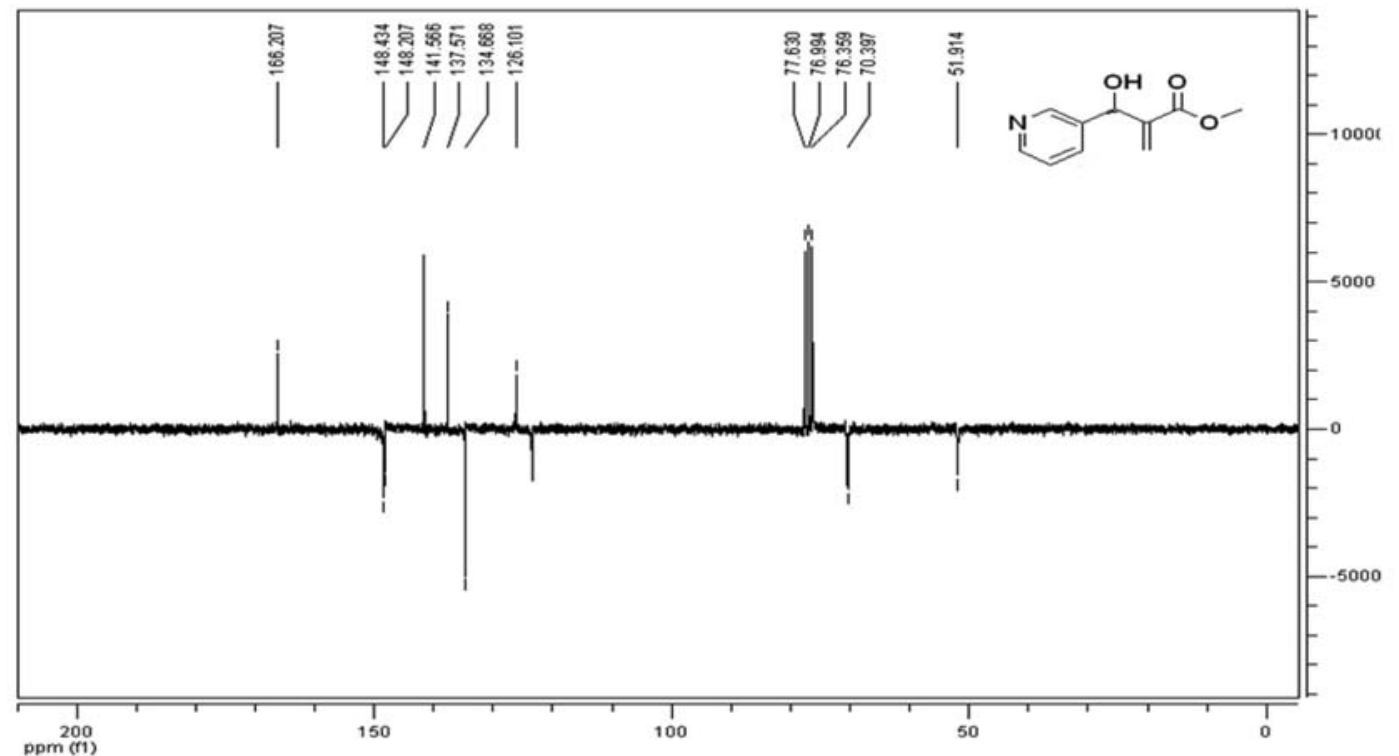

Figure S43. ${ }^{13} \mathrm{C} \mathrm{NMR}\left(\mathrm{CDCl}_{3}\right.$ ) spectrum of Methyl 2-[hydroxyl(3-pyridinyl)methyl] acrylate (6f). 


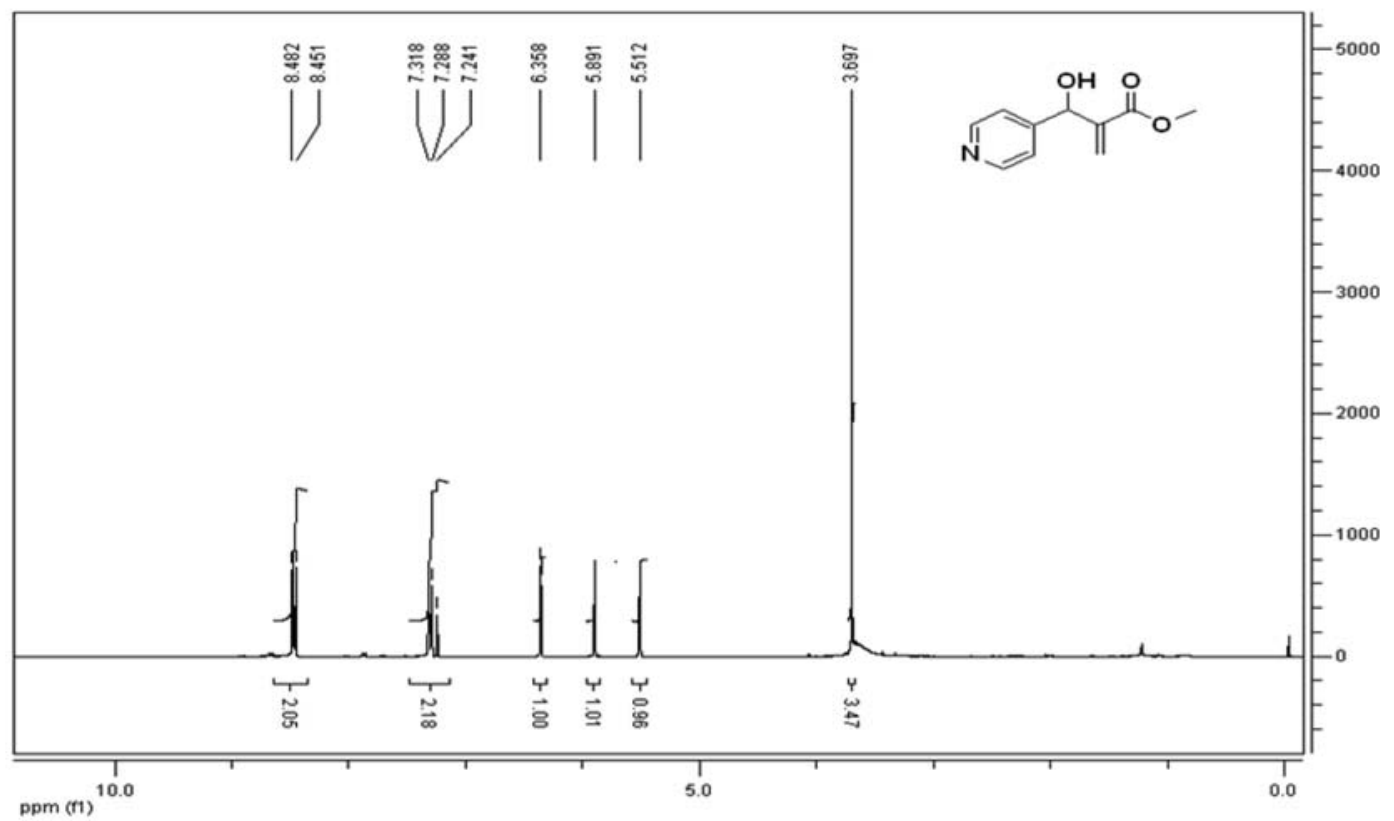

Figure S44. ${ }^{1} \mathrm{H} \mathrm{NMR}\left(\mathrm{CDCl}_{3}\right)$ spectrum of Methyl 2-[hydroxyl (4-pyridinyl)methyl] acrylate $(\mathbf{6 g})$.

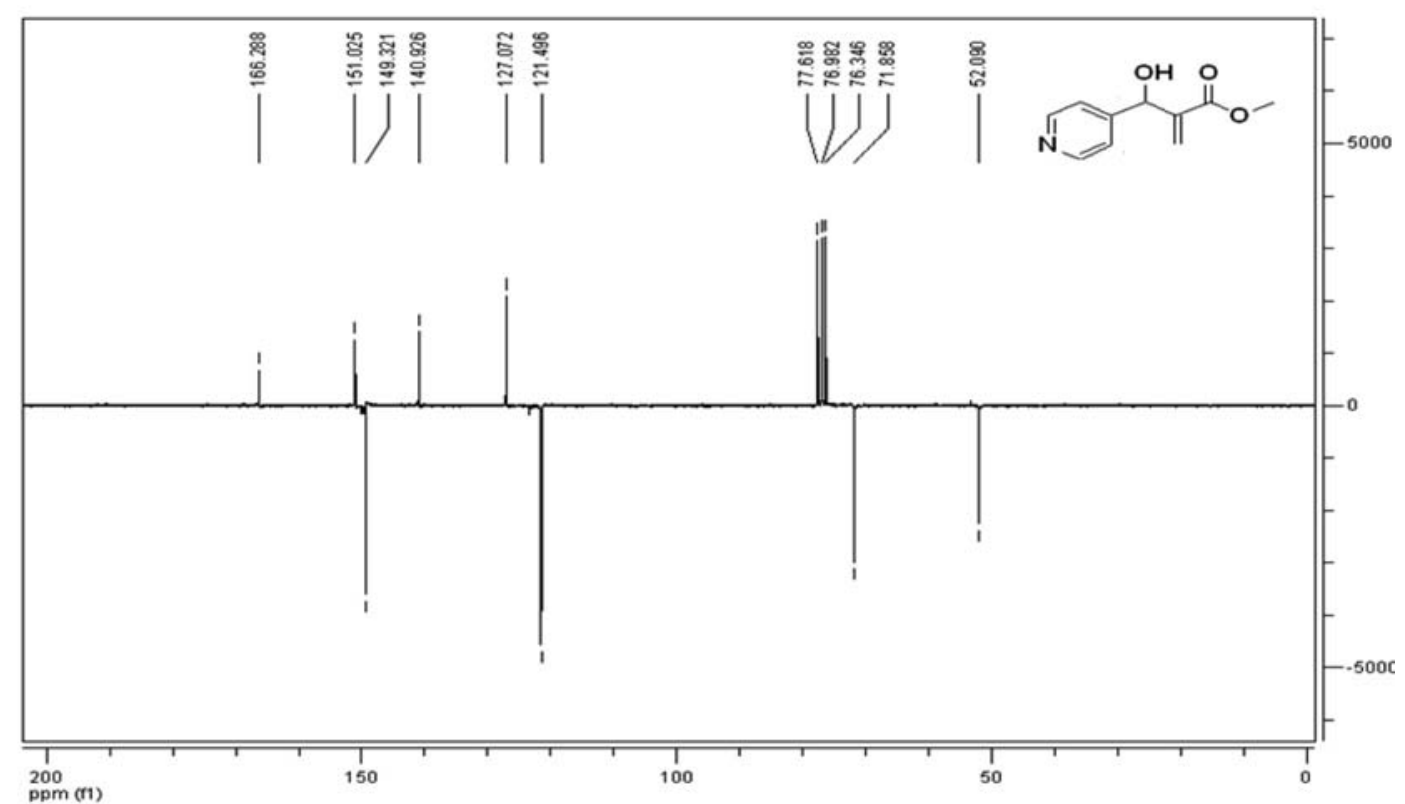

Figure S45. ${ }^{13} \mathrm{C}$ NMR $\left(\mathrm{CDCl}_{3}\right)$ spectrum of Methyl 2-[hydroxyl (4-pyridinyl)methyl] acrylate (6g). 


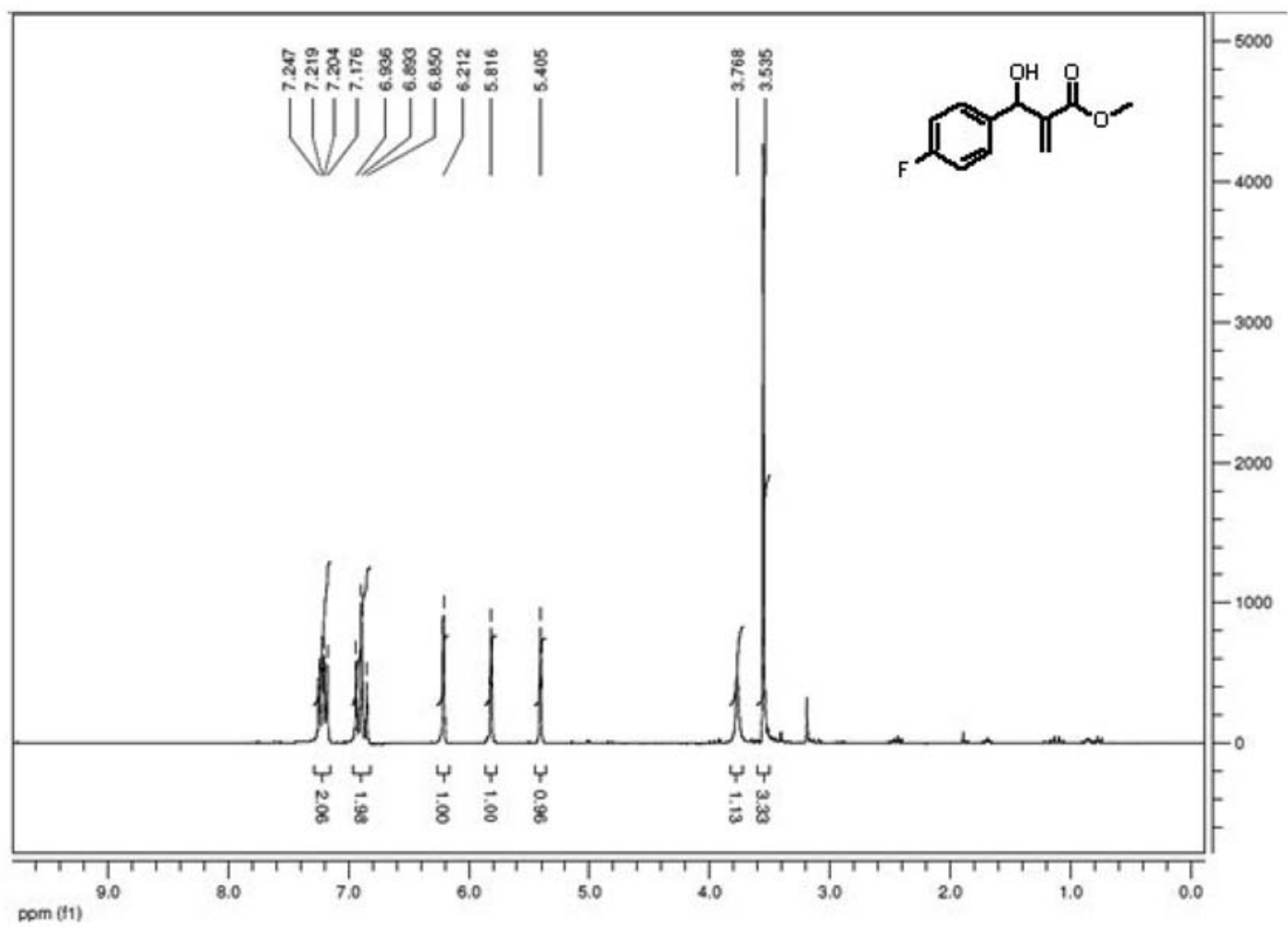

Figure S46. ${ }^{1} \mathrm{H} \mathrm{NMR}\left(\mathrm{CDCl}_{3}\right)$ spectrum of Methyl 2-[hydroxyl (4-fluorphenyl)methyl] acrylate (6h).

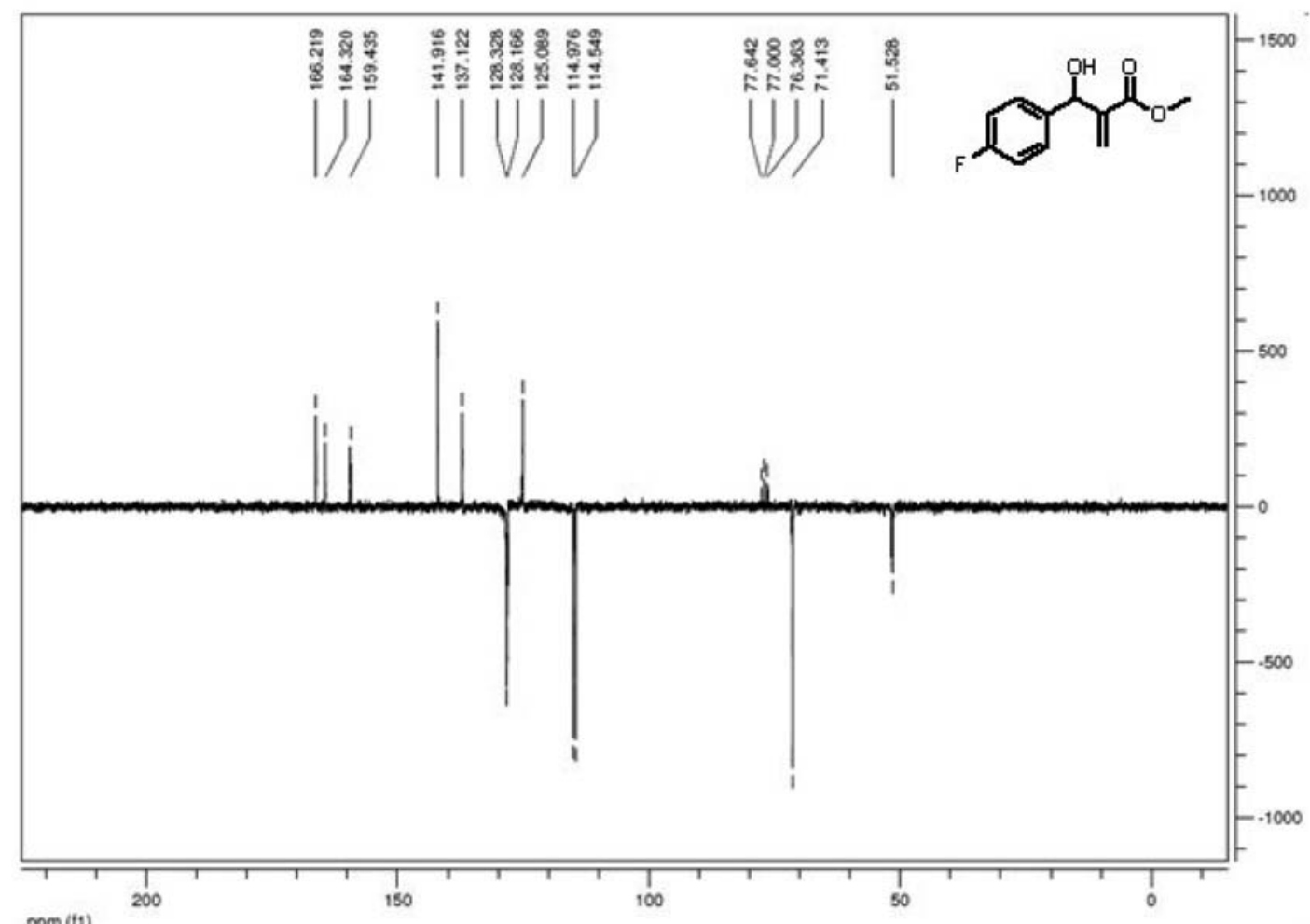

Figure S47. ${ }^{13} \mathrm{C}$ NMR $\left(\mathrm{CDCl}_{3}\right)$ spectrum of Methyl 2-[hydroxyl (4-fluorphenyl)methyl] acrylate (6h). 


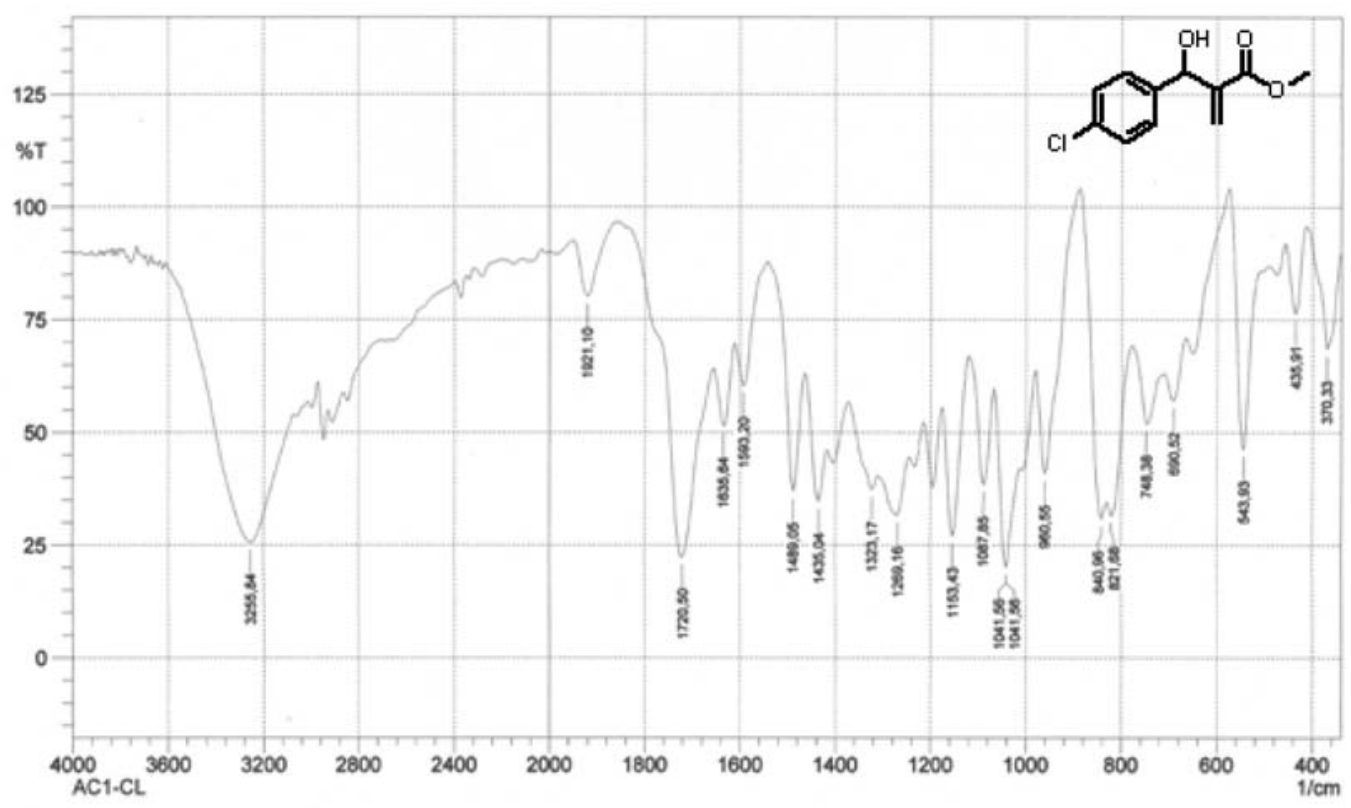

Figure S48. IR (KBr) of Methyl 2-[hydroxyl (4-chlorophenyl)methyl] acrylate (6i).

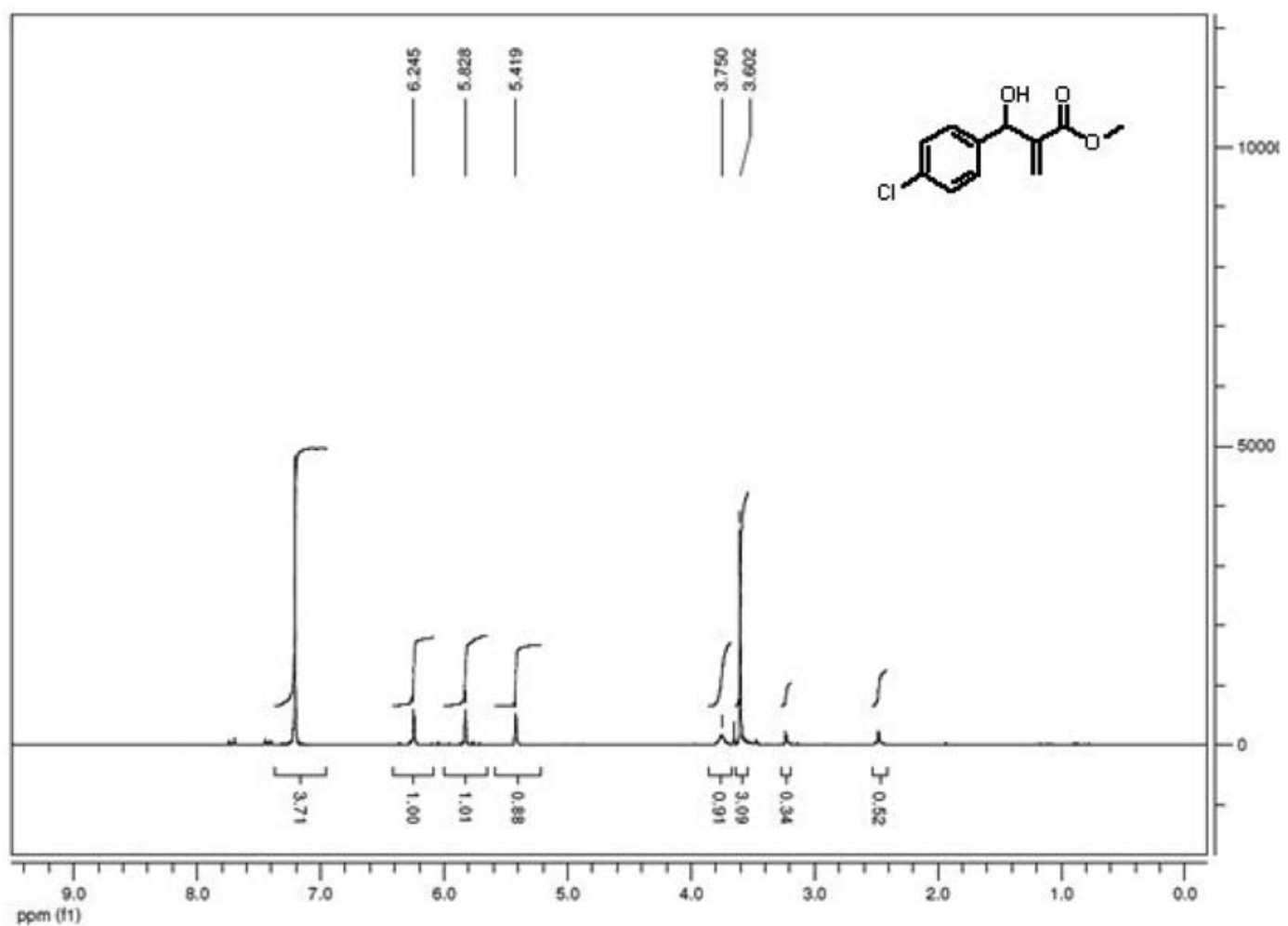

Figure S49. ${ }^{1} \mathrm{H}$ NMR $\left(\mathrm{CDCl}_{3}\right)$ spectrum of Methyl 2-[hydroxyl (4-chlorophenyl)methyl] acrylate (6i). 


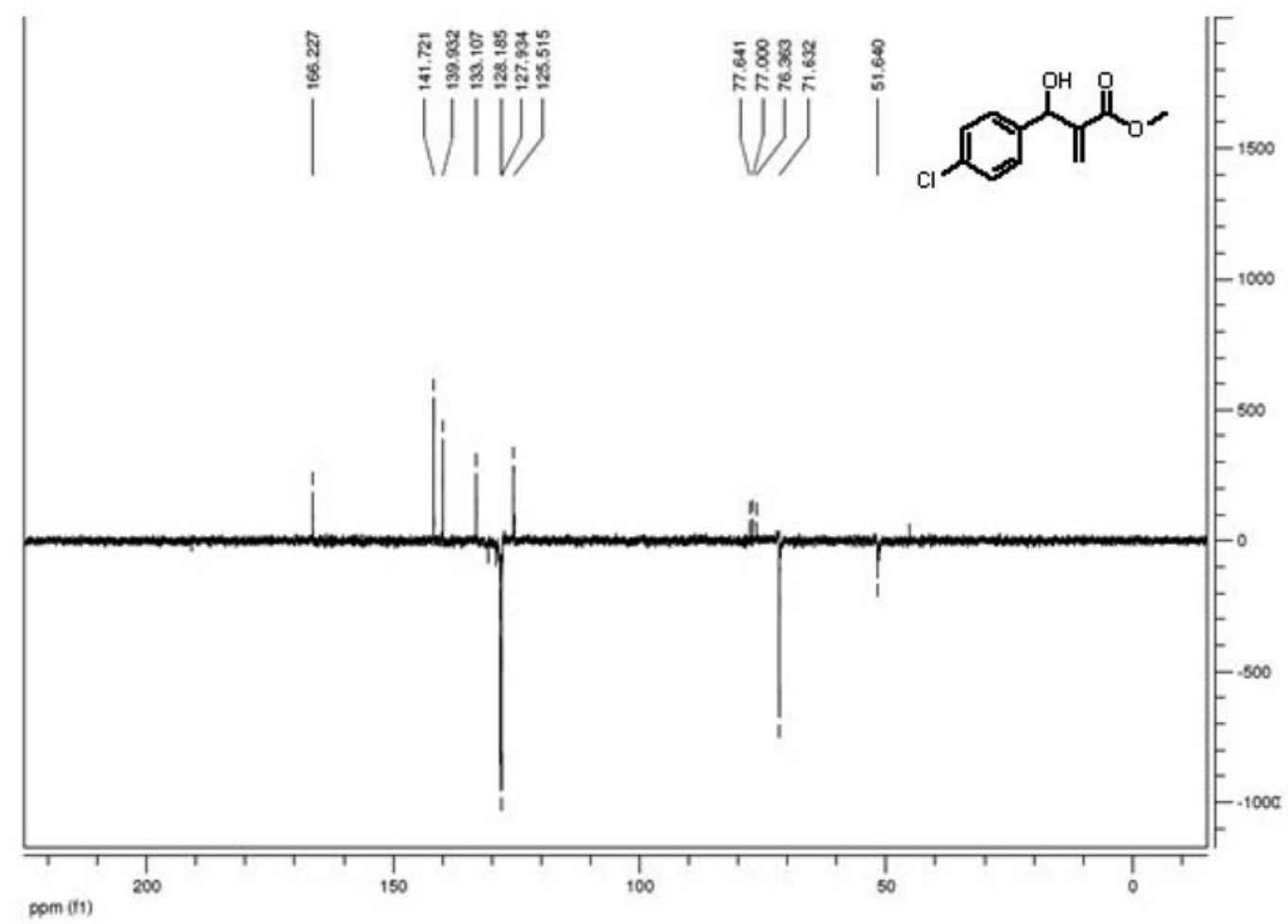

Figure S50. ${ }^{13} \mathrm{C} \mathrm{NMR}\left(\mathrm{CDCl}_{3}\right.$ ) spectrum of Methyl 2-[hydroxyl (4-chlorophenyl)methyl] acrylate (6i).

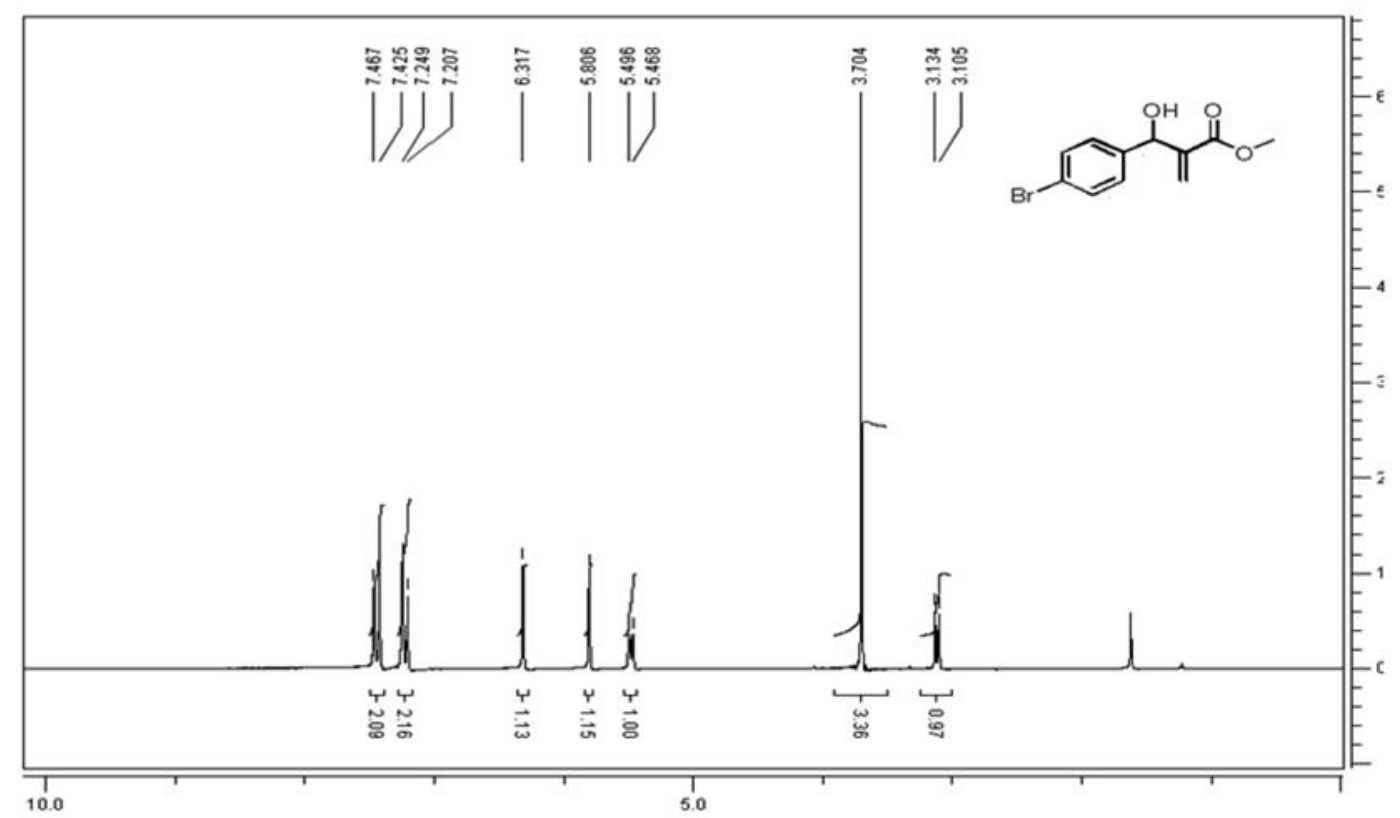

Figure S51. ${ }^{1} \mathrm{H}$ NMR $\left(\mathrm{CDCl}_{3}\right)$ spectrum of Methyl 2-[hydroxyl(4-bromophenyl)methyl] acrylate (6j). 


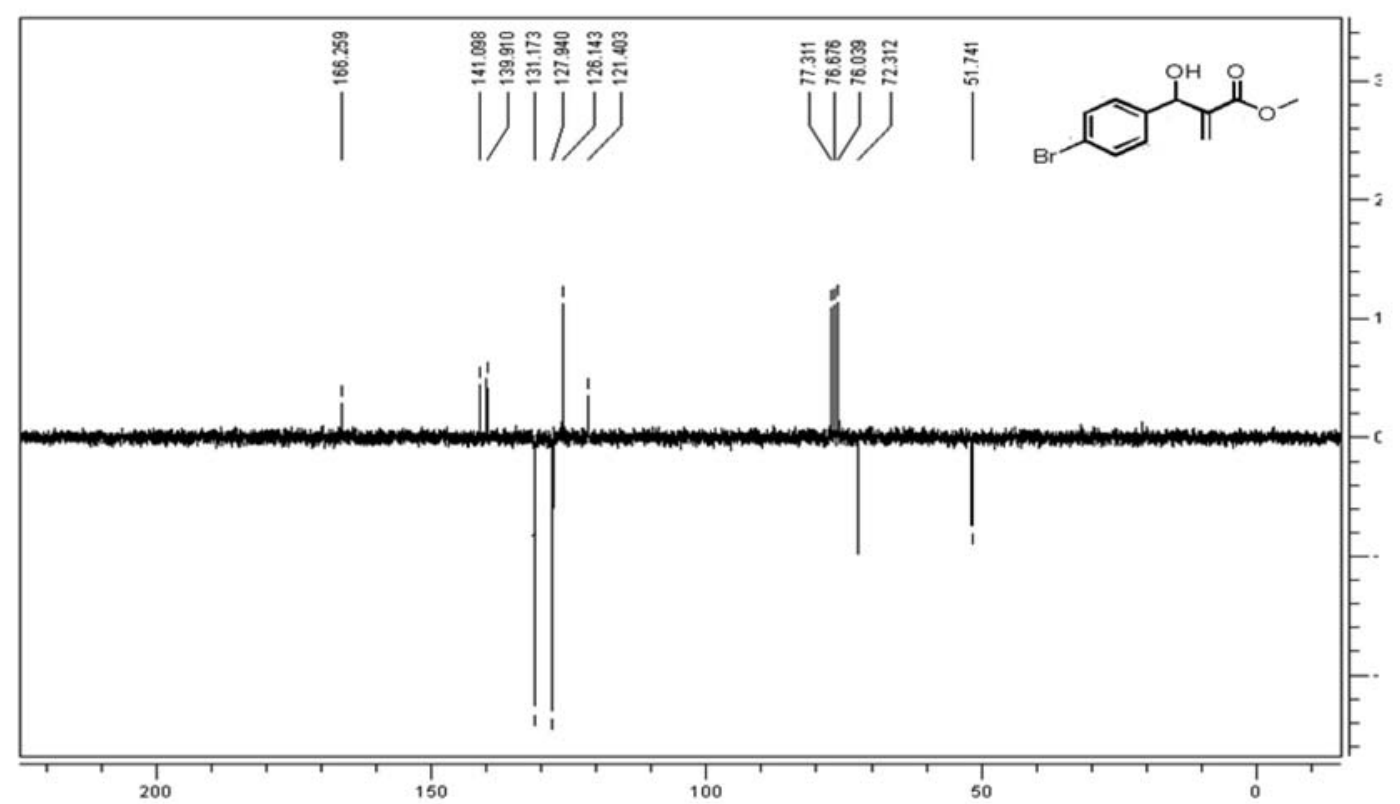

Figure S52. ${ }^{13} \mathrm{C} \mathrm{NMR}\left(\mathrm{CDCl}_{3}\right)$ spectrum of Methyl 2-[hydroxyl(4-bromophenyl)methyl] acrylate (6j).

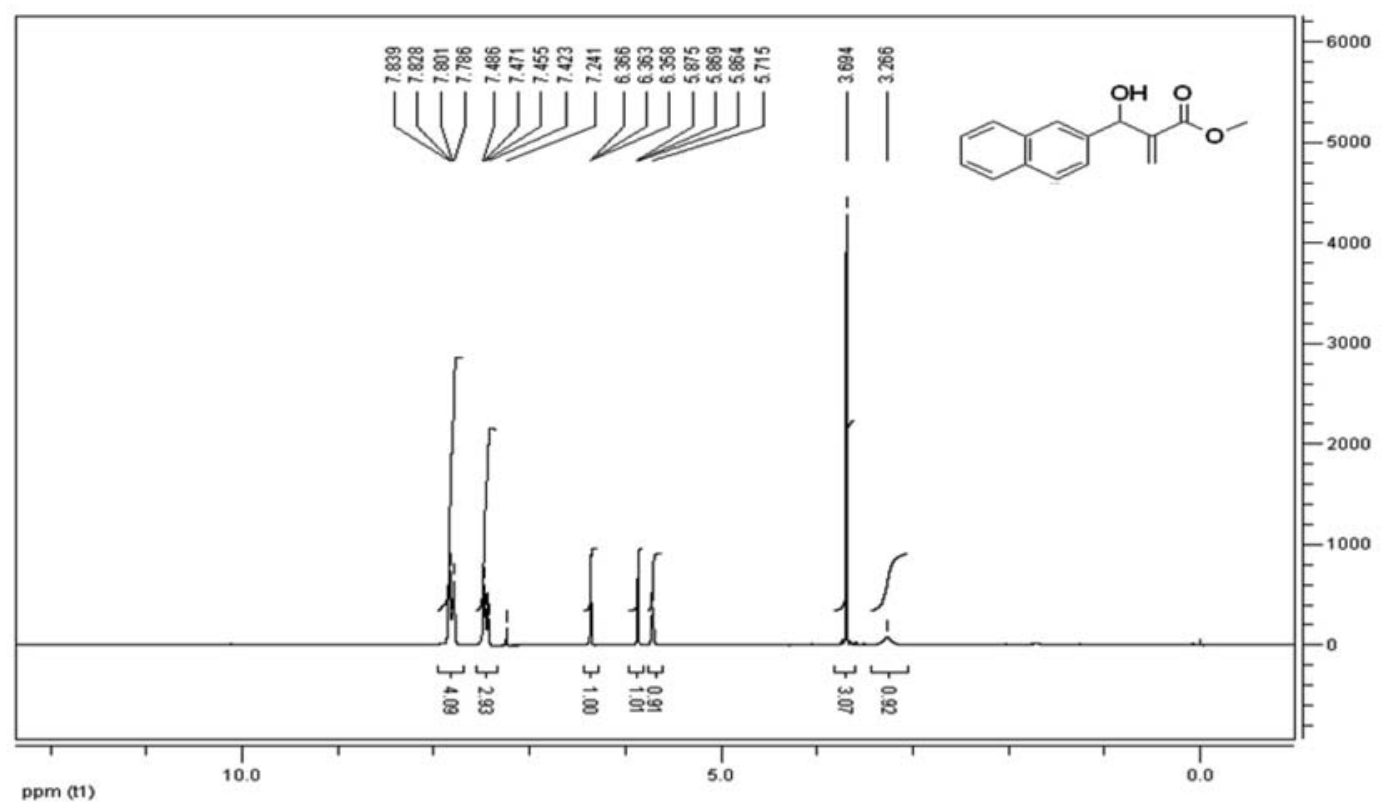

Figure S53. ${ }^{1} \mathrm{H}$ NMR $\left(\mathrm{CDCl}_{3}\right)$ spectrum of Methyl 2-[hydroxyl(2-naphthyl)methyl] acrylate (6k). 


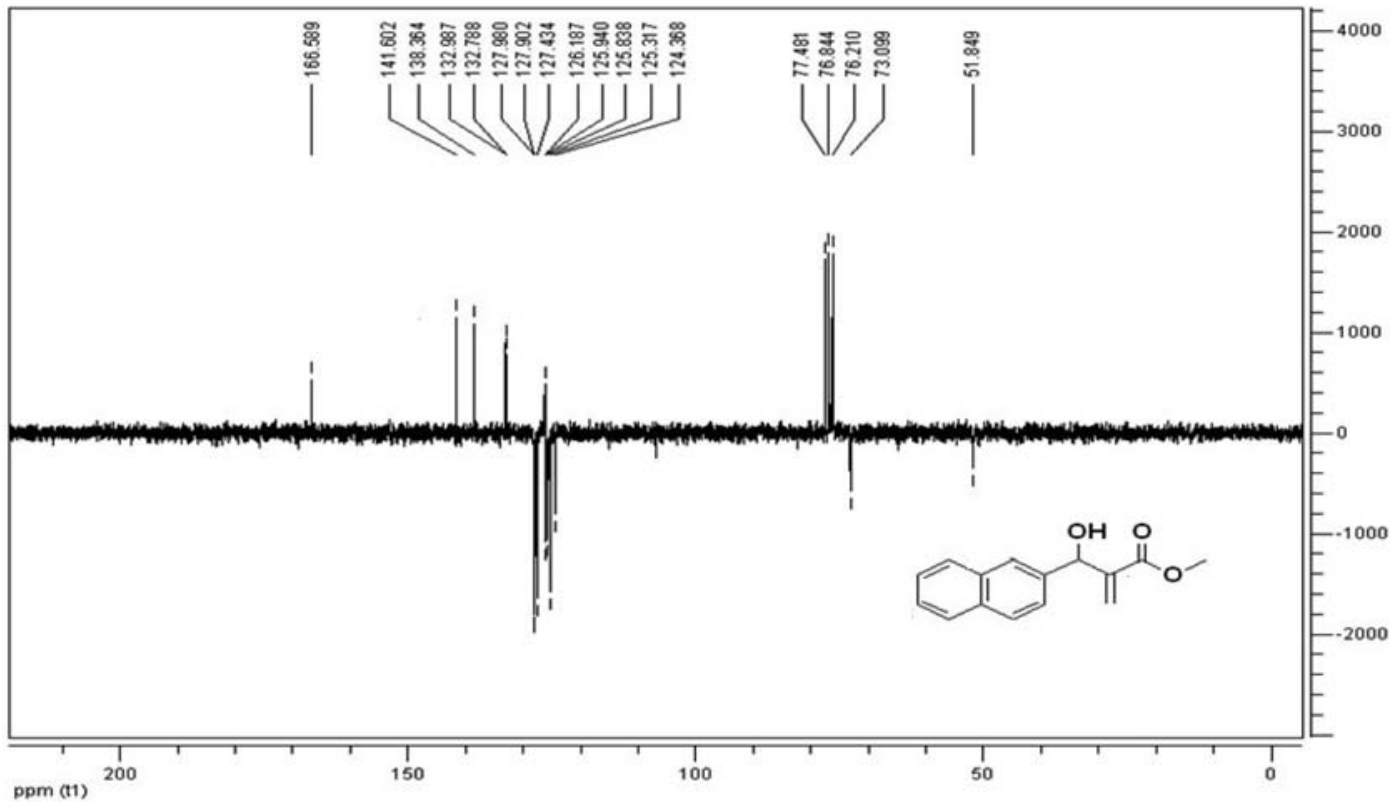

Figure S54. ${ }^{13} \mathrm{C}$ NMR $\left(\mathrm{CDCl}_{3}\right)$ spectrum of Methyl 2-[hydroxyl(2-naphthyl)methyl] acrylate (6k).

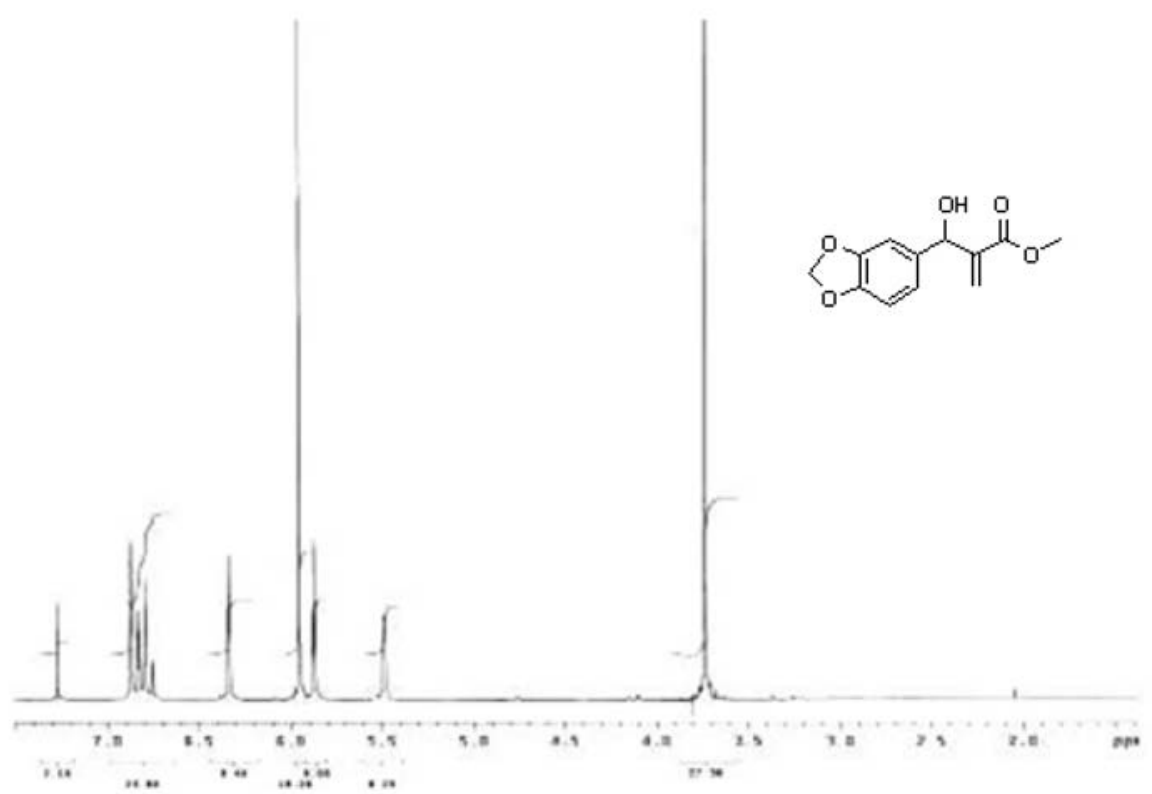

Figure S55. ${ }^{1} \mathrm{H}$ NMR $\left(\mathrm{CDCl}_{3}\right)$ spectrum of Methyl 2-[hydroxyl(1,3-dioxolephenyl)methyl] acrylate (61). 


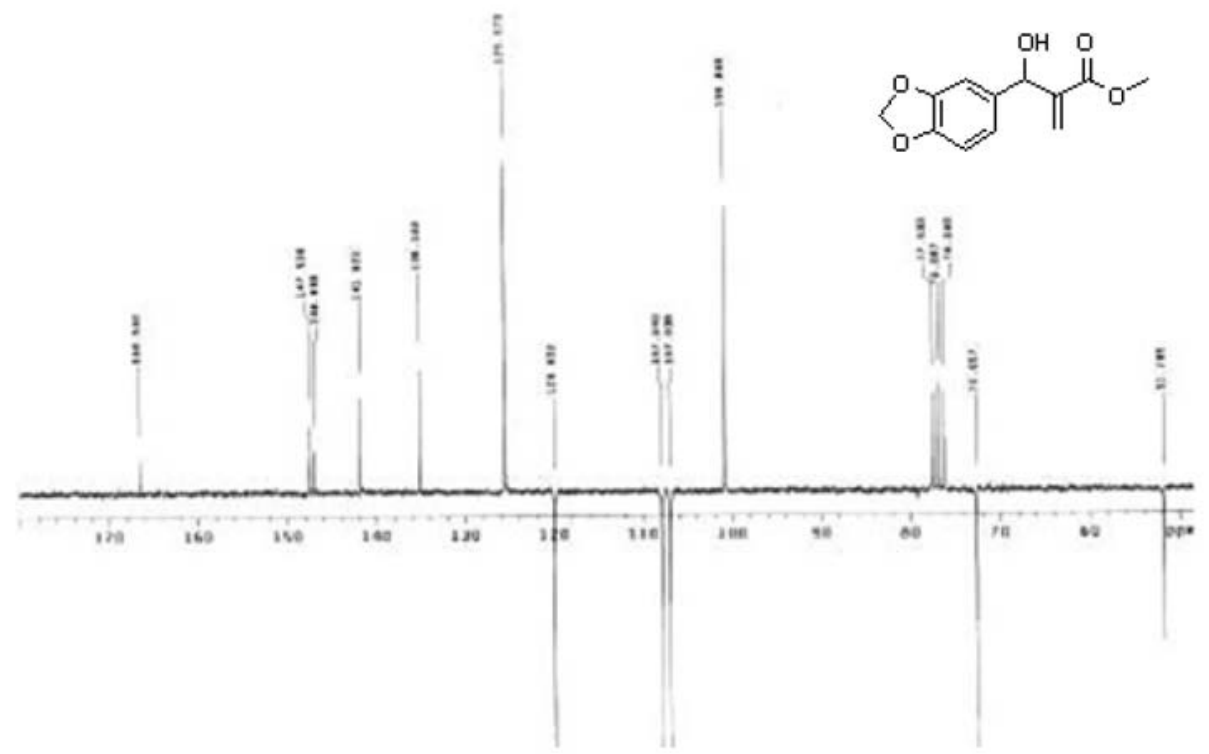

Figure S56. ${ }^{13} \mathrm{C} \mathrm{NMR}\left(\mathrm{CDCl}_{3}\right)$ spectrum of Methyl 2-[hydroxyl(1,3-dioxolephenyl)methyl] acrylate (61). 